\title{
PTP $\sigma$ Drives Excitatory Presynaptic Assembly via Various Extracellular and Intracellular Mechanisms
}

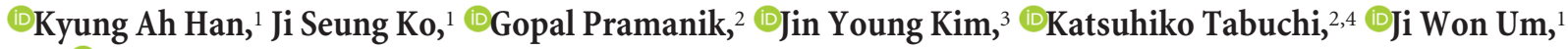 \\ and ${ }^{-J a e w o n ~} \mathrm{Ko}^{1}$ \\ ${ }^{1}$ Department of Brain and Cognitive Sciences, Daegu Gyeongbuk Institute of Science and Technology, Daegu 42988, Korea, ${ }^{2}$ Department of Molecular and \\ Cellular Physiology, Shinshu University School of Medicine, Matsumoto 390-8621, Japan, ${ }^{3}$ Biomedical Omics Group, Korea Basic Science Institute, \\ Cheongju, Chungbuk 28119, Korea, and ${ }^{4}$ Department of Biological Sciences for Intractable Neurological Diseases, Institute for Biomedical Sciences \\ Interdisciplinary Cluster for Cutting Edge Research, Shinshu University, Matsumoto 390-8621, Japan
}

Leukocyte common antigen-receptor protein tyrosine phosphatases (LAR-RPTPs) are hub proteins that organize excitatory and inhibitory synapse development through binding to various extracellular ligands. Here, we report that knockdown (KD) of the LAR-RPTP family member PTP $\sigma$ reduced excitatory synapse number and transmission in cultured rat hippocampal neurons, whereas KD of PTP $\delta$ produced comparable decreases at inhibitory synapses, in both cases without altering expression levels of interacting proteins. An extensive series of rescue experiments revealed that extracellular interactions of PTP $\sigma$ with Slitrks are important for excitatory synapse development. These experiments further showed that the intracellular D2 domain of PTP $\sigma$ is required for induction of heterologous synapse formation by Slitrk1 or TrkC, suggesting that interaction of LAR-RPTPs with distinct intracellular presynaptic proteins, drives presynaptic machinery assembly. Consistent with this, double-KD of liprin- $\alpha 2$ and $-\alpha 3$ or KD of PTP $\sigma$ substrates (N-cadherin and p250RhoGAP) in neurons inhibited Slitrk6-induced, PTP $\sigma$-mediated heterologous synapse formation activity. We propose a synaptogenesis model in presynaptic neurons involving LAR-RPTP-organized retrograde signaling cascades, in which both extracellular and intracellular mechanisms are critical in orchestrating distinct synapse types.

Key words: LAR-RPTPs; presynaptic assembly; protein-protein interaction; PTP $\sigma$; synaptic adhesion molecule

\section{Significance Statement}

In this study, we sought to test the unproven hypothesis that PTP $\sigma$ and PTP $\delta$ are required for excitatory and inhibitory synapse formation/transmission, respectively, in cultured hippocampal neurons, using knockdown-based loss-of-function analyses. We further performed extensive structure-function analyses, focusing on PTP $\sigma$-mediated actions, to address the mechanisms of presynaptic assembly at excitatory synaptic sites. Using interdisciplinary approaches, we systematically applied a varied set of PTP $\sigma$ deletion variants, point mutants, and splice variants to demonstrate that both extracellular and intracellular mechanisms are involved in organizing presynaptic assembly. Strikingly, extracellular interactions of PTP $\sigma$ with heparan sulfates and Slitrks, intracellular interactions of PTP $\sigma$ with liprin- $\alpha$ and its associated proteins through the D2 domain, as well as distinct substrates are all critical.

\section{Introduction}

Synaptogenesis is defined as a continuous process encompassing stabilization of initial contacts between presynaptic axons and

\footnotetext{
Received March 13, 2018; revised May 22, 2018; accepted June 14, 2018.

Author contributions: K.A.H., J.W.U., and J.K. edited the paper; J.K. wrote the first draft of the paper; K.T., J.W.U., and J.K. designed research; K.A.H., J.S.K., G.P., and J.Y.K. performed research; J.W.U. contributed unpublished reagents/analytic tools; K.A.H., J.S.K., G.P., J.Y.K., K.T., J.W.U., and J.K. analyzed data; J.K. wrote the paper.

This work was supported by Korea Healthcare Technology R \& D Project, funded by the Ministry for Health and Welfare Affairs, Republic of Korea Grant HI17C0080 to J.K. We thank Dr. Ho Min Kim (KAIST, Korea) for input in designing various PTP $\sigma$ point mutants.

The authors declare no competing financial interests.

Correspondence should be addressed to Dr. Jaewon Ko, Department of Brain and Cognitive Sciences, Daegu Gyeongbuk Institute of Science and Technology, Daegu 42988, Korea. E-mail: jaewonko@dgist.ac.kr.
}

postsynaptic dendrites, and formation of specialized subcellular machineries near nascent synaptic sites (McAllister, 2007). Earlier studies indicated that individual presynaptic components are preassembled as small numbers of modular units and then transported through the axon via two different vesicle types, synaptic vesicle protein transport vesicles and Piccolo-bassoon transport vesicles, tethered together with microtubule-based motor proteins (Ziv and Garner, 2004; Maas et al., 2012). Both vesicle types are rapidly recruited to, and trapped at, nascent synaptic sites of 
Table 1. List of shRNA constructs

\begin{tabular}{|c|c|c|c|c|}
\hline shRNA construct & Virus type & Target nucleotide sequence $\left(5^{\prime}-3^{\prime}\right)$ & Reference & Full name \\
\hline L-309 sh-PTP $\sigma$ & Lentivirus & GCCACACACCTTCTATAAT & Yim et al., 2013 & Protein tyrosine phosphatase, receptor type, S (Ptprs) \\
\hline L-309 sh-PTP $\delta$ & Lentivirus & GTGCCGGCTAGAAACTTG & Yim et al., 2013 & Protein tyrosine phosphatase, receptor type, D (Ptprd) \\
\hline L-309 sh-LAR & Lentivirus & GCCTACATAGCTACACAG & Yim et al., 2013 & Leukocyte common antigen-related phosphatase (LAR) \\
\hline L-315 sh- $\beta$-catenin & Lentivirus & GCAATCAGCTGGCCTGGTTTG & Current study & $\beta$-Catenin (Ctnnb1) \\
\hline L-315 sh-p250RhoGAP & Lentivirus & ACAAGAAGCACCAAGTA & Nakazawa et al., 2008 & Rho GTPase activating protein 32 (Arhgap32) \\
\hline L-315 sh-Lipirin- $\alpha 2$ & Lentivirus & AGCCAGTCTGATTACAGAA & Current study & PTPRF-interacting protein $\alpha 2$ (Ppfia2) \\
\hline L-315 sh-Lipirin- $\alpha 3$ & Lentivirus & GCTAACATGAAGAAGCTTCAA & Current study & PTPRF-interacting protein $\alpha 3$ (Ppfia3) \\
\hline L-315 sh-N-cadherin & Lentivirus & GGACAACTGTCAGTCACAAAG & Current study & Cadherin 2 \\
\hline
\end{tabular}

synaptogenic signaling (Suarez et al., 2013; Bury and Sabo, 2016). Postsynaptic proteins are also packaged into modular transport packets that are gradually recruited to nascent postsynaptic sites, although postsynaptic protein assembly is relatively slow (Bresler et al., 2004). However, the cellular and molecular mechanisms responsible for coordinating presynaptic and postsynaptic assembly remain unclear (Ziv and Garner, 2004; Jin and Garner, 2008).

Synaptogenic signaling pathways mediated by multiple transsynaptic adhesion molecules are thought to be involved in creating sites of preferential synapse formation (Missler et al., 2012; Um and Ko, 2013; Ko et al., 2015a). Presynaptic neurexins and postsynaptic neuroligins are arguably the most extensively studied pair of synaptogenic trans-synaptic adhesion molecules (Südhof, 2008). Neurexins and neuroligins also bind other synaptic membrane proteins to specify specific features of various types of synapses and neural circuits (Südhof, 2017). Strikingly, neuroligins require extracellular interactions with neurexins, although they do not require neurexin cytoplasmic sequences to induce heterologous synapse formation (Gokce and Südhof, 2013). Thus, neurexins likely use an unidentified presynaptic membrane coreceptor, which subsequently mediates the cytoplasmic signaling cascades required for synapse formation (Gokce and Südhof, 2013). Together with neurexins, leukocyte common antigen-related receptor tyrosine phosphatases (LAR-RPTPs) are also postulated to localize to the presynaptic active zone (Südhof, 2012). LAR-RPTPs, comprising three vertebrate members, LAR, $\mathrm{PTP} \delta$, and $\mathrm{PTP} \sigma$, are evolutionarily conserved cell-surface proteins with tyrosine phosphatase activity that organize synaptic structure and function through binding to a multitude of postsynaptogenic adhesion molecules. Notably, these LAR-RPTP binding partners show no overlap with neurexin-binding proteins, suggesting the existence of distinct intracellular signaling complexes that differ from neurexin-based signaling complexes (Han et al., 2016). Indeed, LAR-RPTPs directly bind to distinct sets of intracellular proteins, including liprins and caskins (Han et al., 2016); however, it is plausible that LAR-RPTPs also indirectly associate via liprin- $\alpha$ with the PDZ-domain proteins, calcium/calmodulin-dependent serine protein $\mathrm{ki}$ nase (CASK) and mammalian synapse-defective-1 protein (mSYD-1), which have been shown to directly interact with neurexins (Um and Ko, 2013). These studies suggest the tantalizing hypothesis that presynaptic neurexins and LARRPTPs organize synapse structure and function through a shared subset of molecular components.

Here, we systematically investigated the effects of LAR-RPTP loss of function on properties of the heterologous synapses formed between cultured neurons and variously transfected HEK293T cells induced by postsynaptic ligands of LAR-RPTPs. Rescue experiments using various deletion variants, point mutants, and splice variants of PTP $\sigma$ revealed that interactions of
PTP $\sigma$ with various postsynaptic ligands through its three immunoglobulin (Ig) domains (Ig1-3) are required for PTP $\sigma$ mediated heterologous synapse-formation activity. Interestingly, reexpression of PTP $\sigma$ phosphatase-inactive mutants incompletely restored heterologous synapse formation in $\mathrm{PTP} \sigma$ deficient neurons, indicating that other mechanisms beyond the intracellular phosphatase activity of $\mathrm{PTP} \sigma$ contribute to this function. Nevertheless, KD of p250RhoGAP (p250GAP) or $\mathrm{N}$-cadherin, but not $\beta$-catenin, blocked formation of heterologous synapses between presynaptic neurons and postsynaptic non-neuronal cells expressing Slitrk6, suggesting that specific $\mathrm{PTP} \sigma$ substrates may act downstream of PTP $\sigma$ to orchestrate the assembly of excitatory presynaptic sites. Furthermore, the carboxy-terminal D2 domain is critical for mediating the synapse-inducing activity of PTP $\sigma$. Together, our results reveal intracellular signaling mechanisms that collectively establish a molecular model of PTP $\sigma$-mediated heterologous synapse formation that differs from that of neurexins.

\section{Materials and Methods}

Construction of expression vectors. 1. Short-hairpin constructs. The indicated shRNA lentiviral expression constructs were generated by annealing, phosphorylating, and subcloning into XhoI/XbaI sites of a KD lentiviral vector (L-309 or L-315) (Pang et al., 2010; Ko et al., 2011). Sequences of oligonucleotides used for cloning of shRNA constructs are presented in Table 1. 2. Rescue constructs. Human PTP $\sigma$ splice variants containing the mini exons encoding $\mathrm{MeA}$ and $\mathrm{MeB}$ sites (but excluding its own signal peptide) were cloned into the pDisplay vector (Invitrogen) via BglII/SalI sites. The PTP $\sigma$ splice variants $\mathrm{PTP} \sigma^{\mathrm{MeA}+\mathrm{MeB}+}$ $\mathrm{PTP} \sigma^{\mathrm{MeA}+\mathrm{MeB}-}, \mathrm{PTP} \sigma^{\mathrm{MeA}-\mathrm{MeB}+}$, and $\mathrm{PTP} \sigma^{\mathrm{MeA}-\mathrm{MeB}-}$ were constructed by amplifying the indicated full-length human PTP $\sigma$ splice variants by PCR, digesting with NheI and BsrGI using the corresponding pDisplay constructs as templates, and subcloning the resulting products into the L-313 vector (lentiviral expression vector). pDisplay-PTP $\sigma$ encoding the full-length human $\mathrm{PTP} \sigma^{\mathrm{MeA}+\mathrm{MeB}+}$ splice variant (GenBank accession number BC143287.1) was used as a template, and all PTP $\sigma$ mutant constructs (except $\Delta \mathrm{FN} 1-2, \Delta$ Cyto, $\Delta \mathrm{D} 2$, and SWAP) were cloned into the pDisplay vector via BglII/SalI sites. shRNA sequences targeting rat $\mathrm{PTP} \sigma$ differ by four nucleotides from the corresponding human PTP $\sigma$ (Yim et al., 2013). Lentiviral expression constructs for rescue experiments were generated by PCR amplification of wild-type (WT) and mutated PTP $\sigma$ WT, followed by digestion with NheI and $B s r G I$ and subcloning into the L-313 vector. The PTP $\sigma / \mathrm{PTP} \delta$ Swap construct was created by PCR amplification of the entire extracellular region of human PTP $\sigma$ (GenBank accession number BC143287.1; aa 30-890) and of mouse PTP $\delta$ (GenBank accession number NM_011211.3; aa 1296-1917) and sequentially cloning the individual PCR fragments into the L-313 vector using an In-Fusion HD cloning kit (Clontech). The original mouse PTP $\delta$ (mPTP $\delta$ ) sequence, 5 '-GTG CCG GCT AGA AAC TTG-3', corresponding to the shRNA target site, was mutated to $5^{\prime}$-GTA CCG GCG AGG AATTTG-3' (altered residues indicated by underlining) in the pcDNA3.1 Myc/His-mPTP $\delta$ (isoform C) vector (a gift from Dr. Fumio Nakamura, Yokohama City University, Japan), and the resulting construct (encoding residues 28-1917 of mPTP $\delta$ ) was PCR-amplified 
and cloned into XmaI/SacII sites in the pDisplay vector (Invitrogen) to create an shRNA-resistant PTP $\delta$ expression construct. An L-313 construct of this shRNA-resistant PTP $\delta$ variant was generated by PCR amplification using pDisplay-PTP $\delta$ as a backbone, and subcloning the resulting product into NheI/BsrGI sites in the L-313 vector. Sequences of oligonucleotides for cloning of various $\mathrm{PTP} \sigma$ and $\mathrm{PTP} \delta$ constructs are presented in Table 2.

Antibodies. The following antibodies were obtained commercially: mouse monoclonal anti-GAD67 (clone 1G10.2; Millipore, RRID: AB_2278725); rabbit polyclonal anti-vesicular glutamate transporter 1 (VGLUT1) (Synaptic Systems, RRID:AB_887880); mouse monoclonal anti-ELKS1 (clone ELKS-30; Sigma-Aldrich, RRID:AB_2100013), and rabbit polyclonal anti-hemagglutinin (HA) (Sigma-Aldrich, RRID: AB_260070); mouse monoclonal anti-CASK (clone K56A/50; NeuroMab, RRID:AB_2068730), mouse monoclonal anti-PSD-95 (clone K28/ 43; NeuroMab, RRID:AB_2307331), and mouse monoclonal antiGluN2B (clone BWJHL; Millipore, RRID:AB_417391); mouse monoclonal anti-PTP $\sigma$ (MediMabs, RRID:AB_1808357); goat polyclonal anti-EGFP (Rockland, RRID:AB_218182); mouse monoclonal anti-HA (clone 16B12; Covance, RRID:AB_2314672); mouse monoclonal anti-Caskin-2 (Santa Cruz Biotechnology, RRID:AB_2713992); and mouse monoclonal anti- $\beta$-actin (clone C4; Santa Cruz Biotechnology, RRID:AB_626632). Rabbit polyclonal anti-Synapsin (JK014, RRID:AB_2651124) was acquired as previously described (Han et al., 2016). The following antibodies were gifts from the indicated investigators: rabbit polyclonal anti-Liprin- $\alpha 2$ and rabbit polyclonal anti-Liprin- $\alpha 3$ (Dr. Susanne Schoch, University of Bonn Medical Center, Bonn, Germany); rabbit polyclonal anti-Caskin-1 antibody (Dr. Katsuhiko Tabuchi, Shinshu University, Nagano Prefecture, Japan); and rat polyclonal anti-PTP $\delta$ antibody (Dr. Fumio Nakamura, Yokohama City University, Yokohama, Japan).

Cell-surface binding assays. Recombinant Fc-fusion proteins of NGL-3 (NGL-3-Fc) and GPC-4 (GPC-4-Fc) were produced in HEK293T cells as previously described (Boucard et al., 2012; Ko et al., 2015b). Soluble $\mathrm{Fc}$-fusion proteins were purified using protein A-Sepharose beads (GE Healthcare). Bead-bound proteins were eluted with $0.1 \mathrm{M}$ glycine, $\mathrm{pH} 2.5$, and then neutralized with $1 \mathrm{~m}$ Tris- $\mathrm{HCl}, \mathrm{pH}$ 8.0. Transfected HEK293T cells expressing HA-tagged PTP $\sigma$ WT or various PTP $\sigma$ variants were incubated with $0.2 \mu \mathrm{M}$ NGL-3-Fc, GPC-4-Fc, or Fc alone (negative control), as indicated. Images were acquired using a confocal microscope (LSM700; Carl Zeiss).

Surface/intracellular protein staining assays in HEK293T cells. HEK293T cells (RRID:CVCL_0063) were transfected with expression vectors for HA-PTP $\sigma$ WT or its various mutants for $24 \mathrm{~h}$. The cells were then washed twice with PBS, fixed with $4 \%$ PFA for $10 \mathrm{~min}$ at $4^{\circ} \mathrm{C}$, blocked with $3 \%$ horse serum $/ 0.1 \%$ BSA (crystalline grade) in PBS for 15 $\mathrm{min}$ at room temperature, and incubated with $1 \mathrm{mg} / \mathrm{ml}$ mouse anti-HA antibody at room temperature. After $1 \mathrm{~h} 30 \mathrm{~min}$, cells were washed twice with PBS and incubated with Cy3-conjugated anti-mouse antibodies (1:500 in blocking solution) for $1 \mathrm{~h}$ at room temperature. Cells were then permeabilized with $0.2 \%$ Triton X-100 in PBS for 10 min at $4^{\circ} \mathrm{C}$, washed, and then incubated with rabbit anti-HA antibody for $1 \mathrm{~h} 30 \mathrm{~min}$ at room temperature to label intracellularly expressed PTP $\sigma$ WT or its mutants. Immunoreactive proteins were detected by incubation with FITCconjugated anti-rabbit secondary antibodies (1:150 in blocking solution). All experiments were repeated three times.

$q R T-P C R$ in cultured neurons. Cultured rat cortical neurons plated on a 12-well plate were treated with the indicated lentiviruses at DIV4. Lentivirus-infected neurons were homogenized at DIV11 in TRIzol Reagent (Invitrogen), and total RNA was extracted according to the manufacturer's protocol. cDNA was prepared from total RNA by random priming using a cDNA synthesis kit (Takara Bio). qRT-PCR was performed using a CFX96 Real-Time PCR system (Bio-Rad) and SYBR Green reagents (Takara Bio). mRNA for the ubiquitously expressed $\beta$-actin was detected as an endogenous control. Sequences of oligonucleotides used for qRT-PCR are presented in Table 3.

Production of recombinant lentiviruses. HEK293T cells were transfected with three plasmids, lentivirus vectors, psPAX2, and pMD2G, at a 2:2:1 ratio using FuGene-6 (Roche Diagnostics), according to the manufacturer's protocol. After $72 \mathrm{~h}$, lentiviruses were harvested by collecting the
Table 2. Overview of PTP $\sigma$ expression constructs

\begin{tabular}{|c|c|c|}
\hline PTP $\sigma$ constructs & Amino acid boundary & Oligo sequence $\left(5^{\prime}-3^{\prime}\right)$ \\
\hline PTP $\sigma^{\mathrm{MeA}+\mathrm{MeB}+}$ & $\operatorname{PTP} \sigma(30-1516)$ & $\begin{array}{l}\text { For pDisplay vector cloning: } \\
\text { Forward: GCG AGATCT GAAGAGCCCCCCAGG } \\
\text { Reverse: GCG GTGGAC TTAGGTTGCATAGTGGTCAAAG } \\
\text { For L-313 vector cloning: } \\
\text { Forward: ATCCGAATTC GCTAGC ATGGAGACAGACACACTCCT } \\
\text { Reverse: CCGCTTTACT TGTACA TTAGGTTGCATAGTGGTCAAAGC }\end{array}$ \\
\hline PTP $\delta$ & $\operatorname{PTP} \delta(28-1917)$ & $\begin{array}{l}\text { For pDisplay vector cloning: } \\
\text { Forward: GGCCAGATCT CCCGGG GAGACACCCCCCAGGT } \\
\text { Reverse: GGACCTGCAG CCGGGGC TACGTTGCATAGTGATCAAA } \\
\text { For L-313 vector cloning: } \\
\text { Forward: ATCCGAATTC GCTAGC ATGGAGACAGACACACTCCT } \\
\text { Reverse: CCGCTTTACT TGTACA CTACGTTGCATAGTGATCAAA }\end{array}$ \\
\hline$\Delta \mathrm{lg}$ & $\operatorname{PTP} \sigma(321-1516)$ & $\begin{array}{l}\text { For pDisplay vector cloning: } \\
\text { Forward: GGG AGATCT CTCCCCAAAGCTCCC } \\
\text { Reverse: GCG GTGGAC TTAGGTTGCATAGTGGTCAAAG } \\
\text { For L-313 vector cloning: } \\
\text { Forward: ATCCGAATTC GCTAGC ATGGAGACAGACACACTCCT } \\
\text { Reverse: CCGCTTACT TGTACA TTAGGTTGCATAGTGGTCAAAGC }\end{array}$ \\
\hline$\Delta \mathrm{FN1}-2$ & $\operatorname{PTP} \sigma(30-335 / 526-1516)$ & $\begin{array}{l}\text { For L-313 vector cloning: 1st fragment } \\
\text { Forward: ATCCGAATTC GCTAGC ATGGAGACAGACACACTCCT } \\
\text { Reverse: CCGCTTACT TGTACA TTTGGGGAGAGATCTAGATTTCAC } \\
\text { For L-313 vector cloning: } 2 \text { nd fragment } \\
\text { Forward: TCTCCCAAA TGTACA GTGCCGGGCCAG } \\
\text { Reverse: CCGCTTACT TGTACA TTAGGTTGCATAGTGGTCAAAGC }\end{array}$ \\
\hline$\Delta$ Ecto & $\operatorname{PTP} \sigma(856-1516)$ & $\begin{array}{l}\text { For pDisplay vector cloning: } \\
\text { Forward: GCG AGATCT GACCCCCAGCCCA } \\
\text { Reverse: GCG GTGGAC TTAGGTTGCATAGTGGTCAAAG } \\
\text { For L-313 vector cloning: } \\
\text { Forward: ATCCGAATTC GCTAGC ATGGAGACAGACACACTCCT } \\
\text { Reverse: CCGCTTACT TGTACA TTAGGTTGCATAGTGGTCAAAGC }\end{array}$ \\
\hline$\Delta$ Cyto & $\operatorname{PTP} \sigma(30-890)$ & $\begin{array}{l}\text { For L-313 vector cloning: } \\
\text { Forward: ATCCGAATTC GCTAGC ATGGAGACAGACACACTCCT } \\
\text { Reverse: CCGCTTTACT TGTACA TTAGTAGAGCAGGATAGCAATGA }\end{array}$ \\
\hline$\Delta \mathrm{D} 2$ & $\operatorname{PTP} \sigma(30-1237)$ & $\begin{array}{l}\text { For L-313 vector cloning: } \\
\text { Forward: ATCCGAATTC GCTAGC ATGGAGACAGACACACTCCT } \\
\text { Reverse: CCGCTTTACT TGTACA TTAGGCCAGCTTCTGGATGTAGGC }\end{array}$ \\
\hline Swap & $\operatorname{PTP} \sigma(30-890) / \operatorname{PTP} \delta(1296-1917)$ & $\begin{array}{l}\text { For L-313 vector cloning: } 1 \text { st fragment } \\
\text { Forward: ATCCGAATTC GCTAGC ATGGAGACAGACACACTCCT } \\
\text { Reverse: CCGCTTACT TGTACA GTAGAGCAGGATAGCAATGACA } \\
\text { For L-313 vector cloning: } 2 \text { nd fragment } \\
\text { Forward: CCTGCTCTAC TGTACA AAAAGGAAGAGGGCAGAGT } \\
\text { Reverse: CCGCTTACT TGTACA CTACGTTGCATAGTGATCAAA }\end{array}$ \\
\hline C1157s & $\operatorname{PTP} \sigma(30-1516)$ & $\begin{array}{l}\text { For mutagenesis: } \\
\text { Forward: CCATCGTGGTTCACAGCAGTGCCGGTGTG } \\
\text { Reverse: CACACCGGCACTGCTGTGAACCACGATGG } \\
\text { For L-313 vector cloning: } \\
\text { Forward: ATCCGAATTCGCTAGC ATGGAGACAGACACACTCCT } \\
\text { Reverse: CCGCTTACT TGTACA TTAGGTTGCATAGTGGTCAAAGC }\end{array}$ \\
\hline R781A & $\operatorname{PTP} \sigma(30-1516)$ & $\begin{array}{l}\text { For mutagenesis: } \\
\text { Forward: GAGGGGCAGCCTGGCGCACTCGGGTCAG } \\
\text { Reverse: CTGACGCGAGTGGGCCAGGCTGCGCCTC } \\
\text { For L-313 vector cloning: } \\
\text { Forward: ATCCGAATTC GCTAGC ATGGAGACAGACACACTCCT } \\
\text { Reverse: CCGCTTTACT TGTACA TTAGGTTGCATAGTGGTCAAAGC }\end{array}$ \\
\hline D1125A & $\operatorname{PTP} \sigma(30-1516)$ & $\begin{array}{l}\text { For mutagenesis: } \\
\text { Forward: CGGCGTGGCCGGCCCATGGCGTGCC } \\
\text { Reverse: GGCACGCCATGGGCCGGCCACGCCG } \\
\text { For L-313 vector cloning: } \\
\text { Forward: ATCCGAATTC GCTAGC ATGGAGACAGACACACTCCT } \\
\text { Reverse: CCGCTTACT TGTACA TTAGGTTGCATAGTGGTCAAAGC }\end{array}$ \\
\hline AAAA & $\operatorname{PTP} \sigma(30-1516)$ & $\begin{array}{l}\text { For mutagenesis: } \\
\text { Forward: GTCTCAAAGCGCTGAGAGTTGACCGCCGCGCCCGCCGCGTTCCAG } \\
\text { GTCACTCGTGGCTTGGG } \\
\text { Reverse: CCCAAGCCACGAGTGACCTGGAACGCGGCGGGCGCGGCGGTCAAC } \\
\text { TCTCAGGGCTTGAGAC } \\
\text { For L-313 vector cloning: } \\
\text { Forward: ATCCGAATTCGCTAGC ATGGAGACAGACACACTCCT } \\
\text { Reverse: CCGCTTTACT TGTACA TTAGGTTGCATAGTGGTCAAAGC }\end{array}$ \\
\hline $\mathrm{R} 97 / 100 \mathrm{~A}$ & $\operatorname{PTP} \sigma(30-1516)$ & $\begin{array}{l}\text { For mutagenesis: } \\
\text { Forward: CAGCCGCTGGCGACACCGGCGGATGAAAACGTGTACG } \\
\text { Reverse: GGTACACGTTTTCATCCGCCGGTGTGCCAGCGGGG } \\
\text { For L-313 vector cloning: } \\
\text { Forward: ATCCGAATTC GCTAGC ATGGAGACAGACACACTCCT } \\
\text { Reverse: CCGCTTACT TGTACA TTAGGTTGCATAGTGGTCAAAGC }\end{array}$ \\
\hline Y233S & $\operatorname{PTP} \sigma(30-1516)$ & $\begin{array}{l}\text { For mutagenesis: } \\
\text { Forward: CACCTGCCAACCTCTCCGTGCGAGAGCTTCG } \\
\text { Reverse: CGAAGCTCTCGCACGGAGAGGTTGGCAGGTG } \\
\text { For L-313 vector cloning: } \\
\text { Forward: ATCCGAATTC GCTAGC ATGGAGACAGACACACTCCT } \\
\text { Reverse: CCGCTTTACT TGTACA TTAGGTTGCATAGTGGTCAAAGC }\end{array}$ \\
\hline R235D & $\operatorname{PTP} \sigma(30-1516)$ & $\begin{array}{l}\text { For mutagenesis: } \\
\text { Forward: CTGCCAACCTCTACGTGGACGAGCTTCGAGAAGTCCG } \\
\text { Reverse: CGGACTTCTCGAAGCTCGTCCACGTAGAGGTTGGCAG } \\
\text { For L-313 vector cloning: } \\
\text { Forward: ATCCGAATTC GCTAGC ATGGAGACAGACACACTCCT } \\
\text { Reverse: CCGCTTTACT TGTACA TTAGGTTGCATAGTGGTCAAAGC }\end{array}$ \\
\hline
\end{tabular}


Table 3. Oligonucleotides for qPCRs

\begin{tabular}{llll}
\hline Gene & Species & Oligo sequence $\left(5^{\prime}\right.$-3') & Reference \\
\hline PTP $\sigma$ & Rattus norvegicus & GAACCGATACGCCAATGCA & Yim et al., 2013 \\
PTP $\delta$ & Rattus norvegicus & GGCGGATTGCAGCATAGG & Yim et al., 2013 \\
LAR & Rattus norvegicus & CCCGATGGCTGAGTACAACA & Yim et al., 2013 \\
$\beta$-Catenin & Rattus norvegicus & GCACTATGGCAGACACCATC & Current study \\
p250RhoGAP & Rattus norvegicus & GACCTGGAAGTGAACAGGT & Current study \\
Liprin- $\alpha 2$ & Rattus norvegicus & CTGCCTCTCTTGAGCCAGATAGA & Current study \\
Liprin- $\alpha 3$ & Rattus norvegicus & CTGCCCCAGTACCGAAGCT & Current study \\
N-cadherin & Rattus norvegicus & TGGAAGGCAATCCACTTAC & Current study \\
Actin & Rattus norvegicus & CCTGGACTTCGAGAATGAGATG & Current study \\
\hline
\end{tabular}

media from transfected HEK293T cells and briefly centrifuging at $1000 \times$ $g$ to remove cellular debris. The filtered media with $5 \%$ sucrose were centrifuged at $\sim 118,000 \times g$ for $2 \mathrm{~h}$, after which supernatants were removed and washed with ice-cold PBS. The virus pellet was resuspended in $80 \mu \mathrm{l}$ of PBS.

Heterologous synapse-formation assays. Heterologous synapseformation assays were performed as previously described (Kang et al., 2016). HEK293T cells were transfected with the indicated expression vectors or EGFP alone (Control) using FuGene (Roche Diagnostics). After $48 \mathrm{~h}$, transfected HEK293T cells were trypsinized, seeded onto cultured hippocampal neurons at DIV9, and then coimmunostained with antibodies against the indicated epitopes and synaptic marker proteins at DIV11. Images were acquired by confocal microscopy (LSM700, Carl Zeiss). For quantification purposes, the contours of transfected HEK293T cells were chosen as the ROI. The fluorescence intensities of synaptic marker puncta, normalized with respect to the area of each HEK293T cell, were quantified for both red and green channels using MetaMorph Software (Molecular Devices, RRID:SCR_002368).

Primary neuronal culture, infections, immunocytochemistry, image acquisition, and analyses. Hippocampal and cortical rat neuron cultures were prepared from embryonic day 18 (E18) rat embryos. Rat cultured neurons at DIV4 were infected with KD lentiviruses alone or together with rescue viruses, and immunostained at DIV14. For immunocytochemistry, cultured rat neurons were fixed with $4 \%$ PFA $/ 4 \%$ sucrose in PBS for 10 min at $4^{\circ} \mathrm{C}$, and permeabilized with $0.2 \%$ Triton $\mathrm{X}-100$ in PBS for $10 \mathrm{~min}$ at $4^{\circ} \mathrm{C}$. Neurons were then blocked with $3 \%$ horse serum $/ 0.1 \%$ BSA in PBS for $15 \mathrm{~min}$ at room temperature and incubated with the indicated primary and secondary antibodies in blocking solution for 90 $\mathrm{min}$ at room temperature. Images of randomly selected neurons were acquired using a confocal microscope (LSM700, Carl Zeiss) with a $63 \times$ objective lens; all image settings were kept constant during image acquisition. $z$ stack images obtained through confocal microscopy were converted to maximal projections, and puncta size and density of the indicated presynaptic marker proteins were analyzed in a blinded manner using MetaMorph software (Molecular Devices).

Semiquantitative immunoblotting in cultured neurons. Cultured cortical rat neurons were prepared and infected with the indicated lentiviruses at DIV4. Neuron lysates were prepared at DIV11 and analyzed by immunoblotting with the indicated primary antibodies. Western blot signals were detected using an enhanced chemiluminescence system (PerkinElmer Life Sciences) and quantified using ImageJ software (Fiji, RRID:SCR_002285). For quantification of Western blot signals, a series of dilutions of neuron lysates was loaded to ensure that the signal intensity of $\beta$-actin (used as an internal control for normalization) changed linearly and in proportion to the amount of loaded protein, and that the intensities of Western blot signals were not saturated.

Electrophysiology. Cell culture electrophysiology was performed as previously described (Ko et al., 2011; Um et al., 2014b). For recording mEPSCs, neurons infected with the indicated lentiviruses were patched with the following internal solution: $90 \mathrm{~mm}$ Cs-gluconate, $10 \mathrm{~mm} \mathrm{CsCl}$, 10 mM HEPES, $10 \mathrm{~mm}$ eEGTA, $5 \mathrm{~mm} \mathrm{NaCl}, 4 \mathrm{~mm} \mathrm{Mg}$-ATP, and $0.3 \mathrm{~mm}$ Na-GTP. For mIPSCs, the internal solution was $100 \mathrm{~mm} \mathrm{CsCl,} 5 \mathrm{~mm}$ $\mathrm{NaCl}, 10$ mм EGTA, 10 mм HEPES, 4 mм Mg-ATP, and 0.3 mм Na-GTP.
The recordings were performed in aCSF with the following composition: $100 \mathrm{~mm} \mathrm{NaCl}, 4 \mathrm{~mm} \mathrm{KCl}, 1 \mathrm{~mm} \mathrm{NaH}_{2} \mathrm{PO}_{4}, 20$ mм HEPES, 30 mm glucose, $2 \mathrm{mM} \mathrm{CaCl}_{2}$, and $1 \mathrm{~mm} \mathrm{MgCl}$. mEPSCs and mIPSCs were recorded at a holding potential of $-70 \mathrm{mV}$ in the presence of $1 \mu \mathrm{M}$ TTX (Ascent Scientific) and $100 \mu \mathrm{m}$ picrotoxin (for mEPSCs) or $1 \mu \mathrm{M}$ TTX and $10 \mu \mathrm{M}$ CNQX (for mIPSCs). The experimenter was blinded to the identity of the infected neurons throughout data collection and analysis. All recordings were digitized at $10 \mathrm{kHz}$ and filtered at $2 \mathrm{kHz}$. Recordings were monitored with an EPC10 double USB patch-clamp amplifier (HEKA Elektronik) and analyzed offline using the Mini Analysis Program (Synaptosoft, RRID:SCR_002184).

Experimental design and statistical analysis. Synaptogenic activity in heterologous synapse-formation assays and synaptic puncta in cultured hippocampal neurons were analyzed using HEK293T cells or cultured neurons infected with the indicated lentiviruses (for details, see figure legends). Data analysis and statistical tests were performed using Prism7 software (GraphPad, RRID:SCR_002798). Data are expressed as mean \pm SEM unless stated otherwise, and significance is indicated with asterisk (compared with a value from control group) or hashtag (compared with a value from experimental group). All experiments were performed using at least three independent cultures, and the normality of data distributions was evaluated using the Shapiro-Wilk test. Data were compared by Student's $t$ test, one-way ANOVA using a nonparametric Kruskal-Wallis test, followed by Dunn's multiple-comparison test for post hoc group comparisons, $t$ test, or Mann-Whitney $U$ test; $n$ values used are indicated in the figure legends. Numbers shown indicate replicates, and tests to determine statistical significance are stated in the text and figure legends depicting results of the respective experiments. A $p$ value $<0.05$ was considered statistically significant, and individual $p$ values are indicated in the respective figure legend.

\section{Results}

PTP $\sigma$ and PTP $\delta$ are selectively required for presynaptic assembly triggered by various postsynaptogenic adhesion molecules

The three members of the LAR-RPTP protein family bind to a host of postsynaptic adhesion molecules, including netrin-G ligand 3 (NGL-3), TrkC, interleukin 1 receptor accessory protein-like 1 (IL1RAPL1), interleukin 1 receptor accessory protein (IL-1RAcP), Slitrks (Slitrk1-6), glypicans (GPC-1-6), SALM3, and SALM5 (Um and Ko, 2013; Han et al., 2016). Previous studies have indicated that (1) PTP $\delta$ is required for IL1RAPL1- and IL-1RAcP-triggered excitatory heterologous synapse formation; (2) PTP $\delta$ and PTP $\sigma$ are required for Slitrkinduced inhibitory and excitatory heterologous synapse formation, respectively; (3) LAR, $\mathrm{PTP} \delta$, and $\mathrm{PTP} \sigma$ are required for SALM3-induced heterologous synapse formation; and (4) PTP $\sigma$ is required for SALM5-induced heterologous synapse formation (Valnegri et al., 2011; Yoshida et al., 2011; Takahashi et al., 2012; Yim et al., 2013; Li et al., 2015; Choi et al., 2016). However, whether proteins from the LAR-RPTP family are also required for NGL-3- or TrkC-induced heterologous synapse formation has not been tested. Thus, we first sought to determine which LAR-RPTPs are critical for NGL-3- and TrkC-dependent heterologous synapse formation. To this end, we cocultured HEK293T cells expressing NGL-3 or TrkC with hippocampal neurons infected with lentiviruses expressing an empty shRNA vector (shControl) or an shRNA KD construct targeting LAR (sh-LAR), PTP $\delta$ (sh-PTP $\delta$ ), or PTP $\sigma$ (sh-PTP $\sigma$ ) for $2 \mathrm{~d}$ and performed a series of heterologous synapse formation assays using VGLUT1 as a marker to visualize excitatory presynaptic sites. We found that PTP $\sigma$ KD (sh-PTP $\sigma$ ) impaired heterologous excitatory synapse formation induced by $\operatorname{TrkC}$, consistent with the specific interaction of TrkC with PTP $\sigma$ (Fig. $1 A, B$ ) (Takahashi et al., 2011). In addition, $\mathrm{PTP} \sigma \mathrm{KD}($ sh-PTP $\sigma)$ specifically impaired heterologous excitatory synapse formation triggered by Slitrk1, 
A

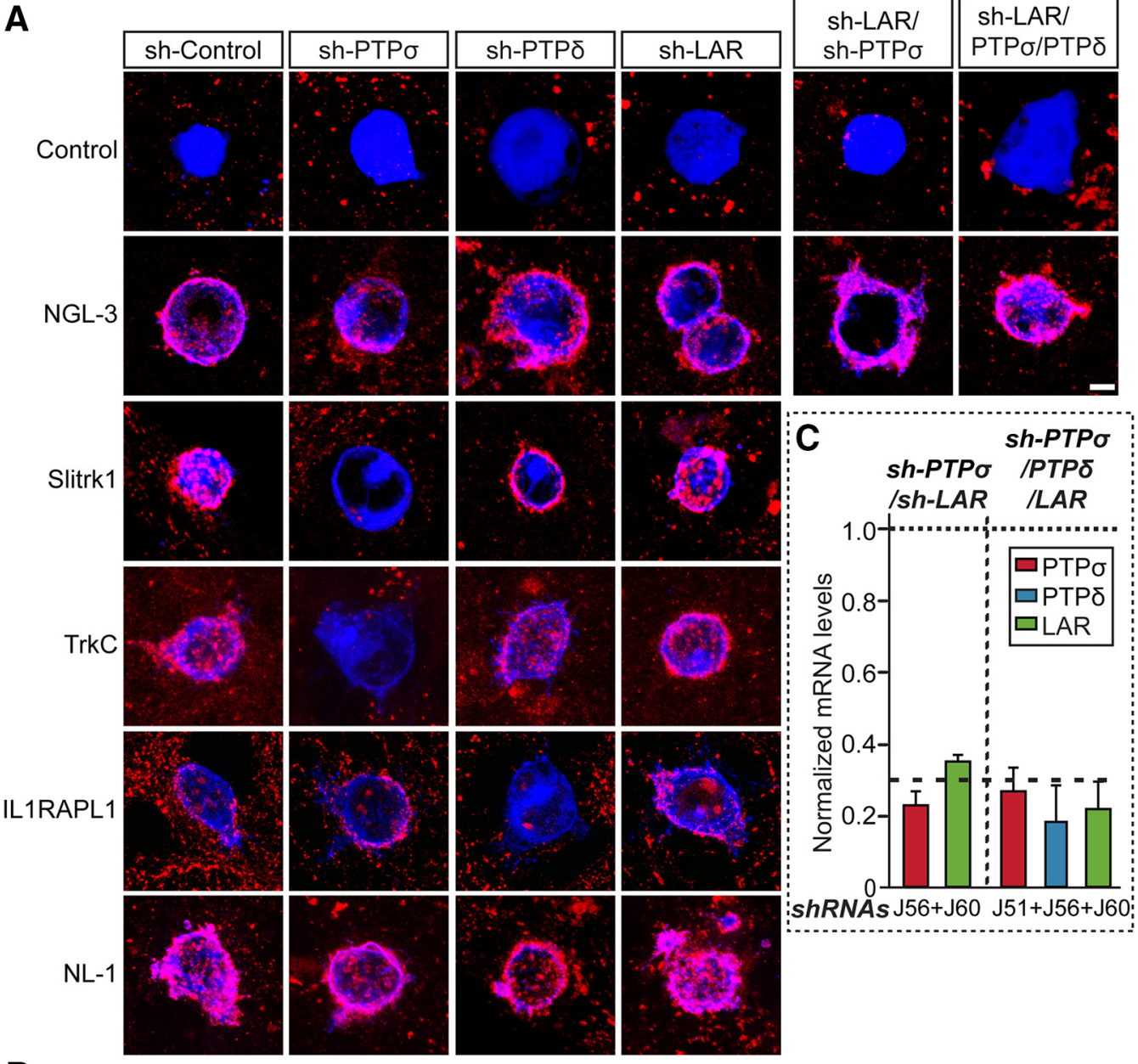

B

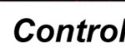

NGL-3

Slitrk1

TrkC

IL1RAPL1 NL-1

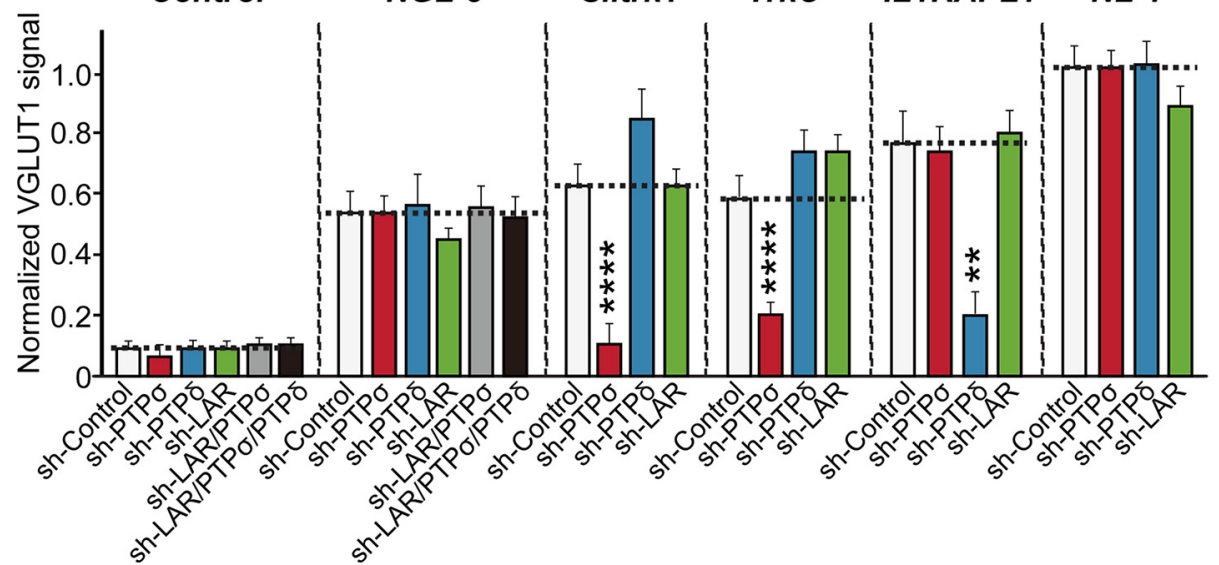

Figure 1. PTP $\sigma$ and PTP $\delta$ are selectively required for the heterologous synapse-formation activities of distinct postsynaptogenic adhesion molecules. $A$, Representative images of the heterologous synapse-formation activities of various LAR-RPTP ligands. Neurons were infected at DIV4 with lentiviruses expressing sh-Control, sh-PTP $\sigma$, sh-PTP $\delta$, sh-LAR, sh-LAR/sh-PTP $\sigma$, or sh-PTP $\sigma /$ PTP $\delta / L A R$ as indicated, and then cocultured from DIV9 to DIV11 with HEK293T cells expressing various postsynaptic ligands. Neurons were stained with antibodies against HA or EGFP (blue) and VGLUT1 (red). Scale bar (all images), $10 \mu \mathrm{m} . \boldsymbol{B}$, The synapse-forming activity in $\boldsymbol{A}$ was quantified by measuring the ratio of VGLUT1 staining intensity (red) to HA or EGFP immunoreactivity intensity (blue). Data are mean \pm SEM. Mann-Whitney $U$ test: ${ }^{* * *} p<0.0001$. $n=$ number of neurons as follows: sh-Control/Control, $n=13$; sh-PTP $\sigma /$ Control, $n=15 ;$ sh-PTP $\delta /$ Control, $n=17$; sh-LAR/Control, $n=15 ;$ sh-LAR/sh-PTP $\sigma /$ Control, $n=11 ;$ sh-LAR/PTP $\sigma /$ PTP $\delta /$ Control, $n=12 ;$ sh-Control/NGL-3, $n=18 ;$ sh-PTP $\sigma /$ NGL-3, $n=17 ;$ sh-PTP $\delta /$ NGL-3, $n=16 ;$ sh-LAR/NGL-3, $n=$ 16; sh-LAR/sh-PTP $\sigma /$ NGL-3, $n=16$; sh-LAR/PTP $\sigma /$ PTP $\delta /$ NGL-3, $n=16$; sh-Control/Slitrk1, $n=13$; sh-PTP $\sigma / S l i t r k 1, n=15 ;$ sh-PTP $\delta /$ Slitrk1, $n=13$; sh-LAR/Slitrk1, $n=11 ;$ sh-Control/TrkC, $n=15 ;$ sh-PTP $\sigma / \operatorname{TrkC}, n=11 ;$ sh-PTP $\delta /$ TrkC, $n=12 ;$ sh-LAR/TrkC, $n=11$; sh-Control/LL1RAPL1, $n=11$; sh-PTP $\sigma /$ LL1RAPL1, $n=13 ;$ sh-PTP $\delta / L 1$ RAPL1, $n=14 ;$ sh-LAR/IL1RAPL1, $n=12 ;$ sh-Control/NL-1, $n=11$; sh-PTP $\sigma /$ NL-1, $n=12$; sh-PTP $\delta / N L-1, n=10 ;$ and sh-LAR/NL-1, $n=11$. $p$ values for Control condition: sh-Control vs sh-PTP $\sigma, p=0.439 ;$ sh-Control vs sh-PTP $\delta, p=$ 0.5604; sh-Control vs sh-LAR, $p=0.1835$; sh-Control vs sh-LAR/sh-PTP $\sigma, p=0.9534$; and sh-Control vs sh-LAR/PTP $\sigma / P T P \delta, p=0.5641 . p$ values for NGL-3 condition: sh-Control vs sh-PTP $\sigma, p=$ 0.4129 ; sh-Control vs sh-PTP $\delta, p=0.7706$; sh-Control vs sh-LAR, $p=0.5832$; sh-Control vs sh-LAR/sh-PTP $\sigma, p=0.8166$; and sh-Control vs sh-LAR/PTP $\sigma / P T P \delta, p=0.9921 . p$ values for Slitrk1 condition: sh-Control vs sh-PTP $\sigma, p<0.0001$; sh-Control vs sh-PTP $\delta, p=0.3394$; and sh-Control vs sh-LAR, $p=0.684$. $p$ values for TrkC condition: sh-Control vs sh-PTP $\sigma, p<0.0001$; sh-Control vs sh-PTP $\delta, p=0.1433$; and sh-Control vs sh-LAR, $p=0.2958$. $p$ values for IL1RAPL1 condition: sh-Control vs sh-PTP $\sigma, p=0.7832$; sh-Control vs sh-PTP $\delta, p<0.01$; and sh-Control vs sh-LAR, $p=0.5212$. $p$ values for NL1 condition: sh-Control vs sh-PTP $\sigma, p=0.9537$; sh-Control vs sh-PTP $\delta, p=0.8919$; and sh-Control vs sh-LAR, $p=0.6262$. C, Levels of PTP $\sigma$, PTP $\delta$, and LAR mRNAs were measured at DIV12-DV13 by qRT-PCR in cultured cortical neurons infected at DIV4 with lentiviruses expressing the indicated shRNAs. Dashed line indicates $70 \%$ knockdown cutofflevel for tests of biological effects. 

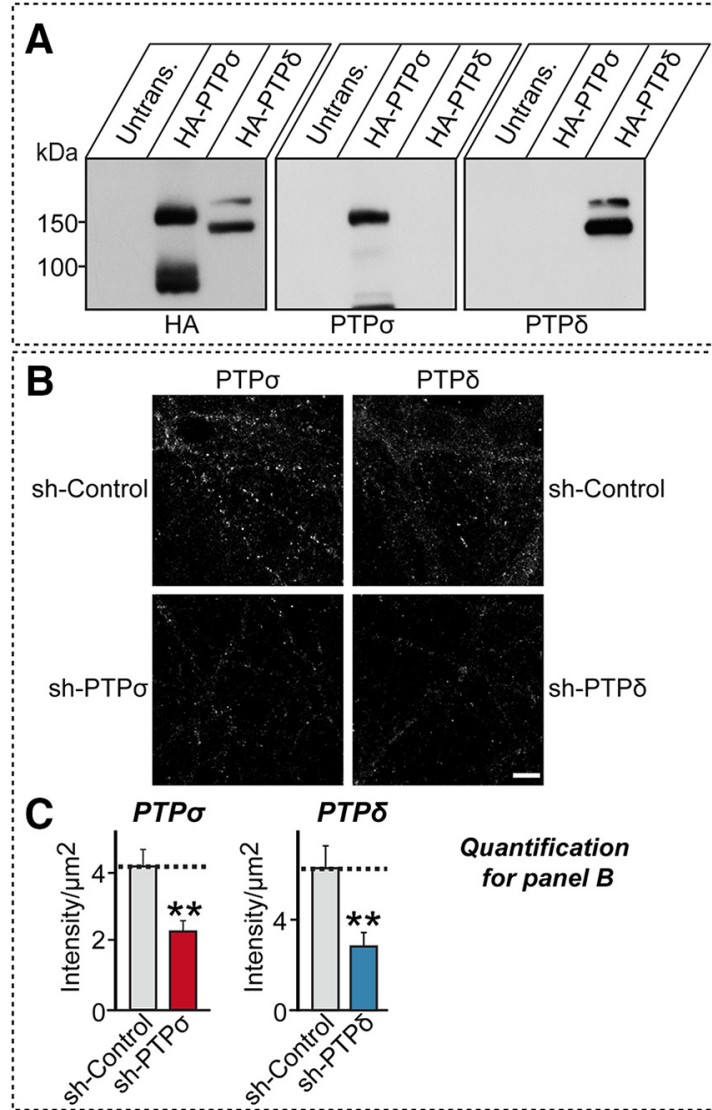

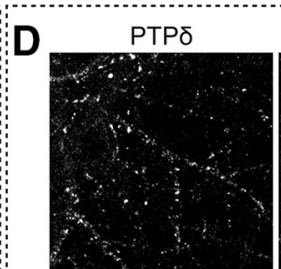

РТРठ
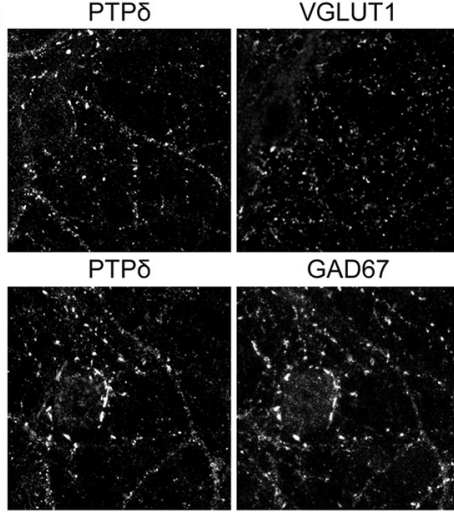

GAD67
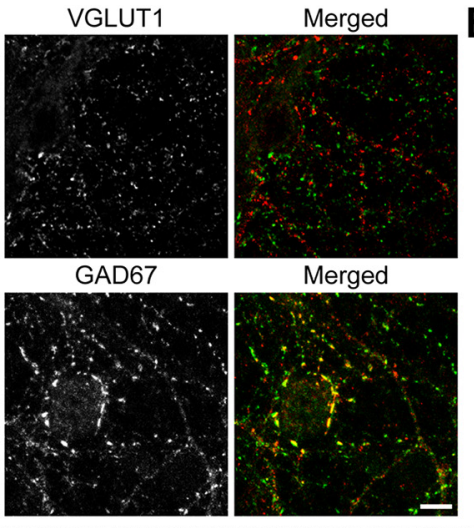

$\mathbf{F}$

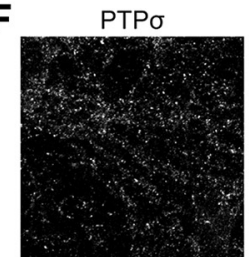

PTPo
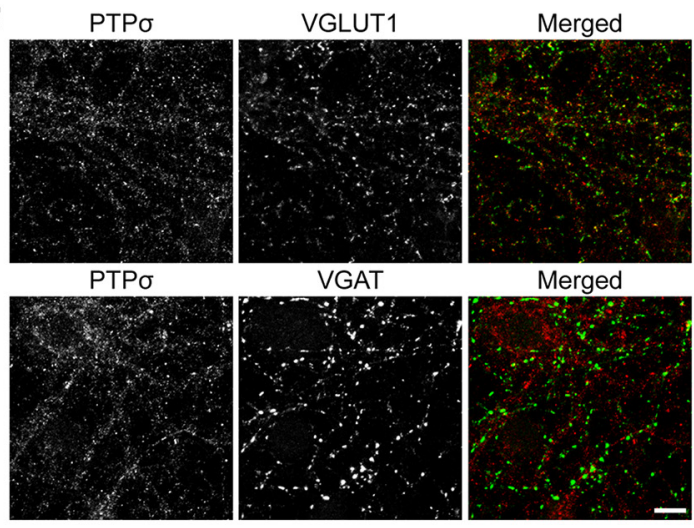

G

Quantification for panel $F$

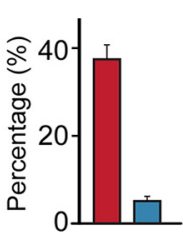

$\square$ VGLUT1-positive

PTPo puncta

$\square$ VGAT-positive

PTPo puncta

Figure 2. Specific localization of PTP $\sigma$ and PTP $\delta$ at excitatory and inhibitory synaptic sites in cultured hippocampal neurons. A, Immunoblot analyses of PTP $\delta$ and PTP $\sigma$ using lysates from HEK293T cells transfected with HA-tagged PTP $\delta$ (HA- PTP $\delta$ ) or PTP $\sigma$ (HA-PTP $\sigma$ ). The expression of HA-tagged PTP isoforms was confirmed by immunoblotting with anti-HA antibodies. Unt., Untransfected HEK293T cell lysates; Trans. (HA-PTP $\delta$ ), lysates from HEK293T cells transfected with HA-PTP $\delta$; Trans. (HA-PTP $\sigma$ ), lysates from HEK293T cells transfected with HA-PTP $\sigma . B$, Authenticity testing of anti-PTP $\delta$ and anti-PTP $\sigma$ antibodies. Mature cultured hippocampal neurons (DIV14) infected with lentiviruses expressing sh-Control, sh-PTP $\delta$ (PTP $\delta$ KD), or sh-PTP $\sigma($ PTP $\sigma$ KD) were labeled by single immunofluorescence staining for the indicated PTP antibodies (gray). Scale bar (all images), $10 \mu \mathrm{m}$. $\boldsymbol{C}$, Summary graphs of $\boldsymbol{B}$. Data are mean \pm SEM. ${ }^{* *} p<0.01$ (Mann-Whitney $U$ test). $n=$ number of analyzed neurons as follows: sh-Control/PTP $\sigma, n=10$; sh-PTP $\sigma /$ PTP $\sigma, n=10 ;$ sh-Control/PTP $\delta, n=10$; and sh-PTP $\delta /$ PTP $\delta, n=11 . p$ value for PTP $\sigma$ antibody: sh-Control vs sh-PTP $\sigma, p=0.0052$. $p$ value for PTP $\delta$ antibody: sh-Control vs sh-PTP $\delta, p=0.0048$. D, $\boldsymbol{F}$, Immunolocalization of PTP $\delta(\boldsymbol{D})$ and PTP $\sigma(\boldsymbol{F})$ in cultured hippocampal neurons, along with colocalization of GAD67, VGAT, or VGLUT1 as indicated. Scale bar (all images), $10 \mu \mathrm{m} . \boldsymbol{E}, \mathbf{G}$, Quantification of colocalization of endogenous PTP $\delta(\boldsymbol{E})$ and $\operatorname{PTP} \sigma(\boldsymbol{G})$ with synaptic markers.

whereas PTP $\delta$ KD (sh-PTP $\delta$ ) specifically impaired heterologous excitatory synapse formation induced by IL1RAPL1 (Fig. 1 $A, B$ ) (Valnegri et al., 2011; Yoshida et al., 2011; Yim et al., 2013). Remarkably, KD of individual LAR-RPTP family proteins (shPTP $\sigma$, sh-PTP $\delta$, or sh-LAR) did not impair NGL-3-induced heterologous synapse formation (Fig. $1 A, B$ ). Surprisingly, KD of all three LAR-RPTPs (sh-PTP $\sigma$, PTP $\delta$, LAR) had no effect on NGL-3-mediated heterologous excitatory synapse formation (Fig. 1). In control experiments, heterologous synapse formation induced by neuroligin-1 (NL-1) was not affected by KD of individual LAR-RPTPs (Fig. $1 A, B$ ). Together, these data indicate that $\mathrm{PTP} \sigma$ and $\mathrm{PTP} \delta$ are distinctively required for heterologous excitatory synapse formation mediated by LARRPTP-interacting postsynaptic ligands.

PTP $\sigma$ and PTP $\delta$ are selectively required for excitatory and inhibitory synapse structure/transmission, respectively, in cultured hippocampal neurons

Recombinant PTP $\sigma$ and PTP $\delta$ were previously reported to localize to excitatory and inhibitory synaptic sites, respectively, in cultured hippocampal neurons (Takahashi et al., 2011, 2012); however, the subcellular localization of endogenous PTP $\delta$ in cultured neurons was not assessed (Takahashi et al., 2012). There- fore, we examined whether endogenous $\mathrm{PTP} \delta$ specifically localizes to inhibitory synaptic sites. The PTP $\sigma$ and PTP $\delta$ antibodies used in the current study exhibited no cross-reactivities, demonstrating specificity for the target antigen (Fig. $2 A$ ). The authenticity of these antibodies was further validated by showing that immunoreactivity to them was diminished in cultured hippocampal neurons infected with a KD virus targeting the corresponding protein (Fig. $2 B, C$ ). Using these validated antibodies, we found that PTP $\delta$ immunoreactivity was present in a punctate pattern decorating the dendrites of cultured hippocampal neurons at DIV14; these puncta overlapped with glutamate decarboxylase 1 (GAD67) by $\sim 48 \%$, but with VGLUT1 by only $\sim 10 \%$ (Fig. $2 D, E$ ). PTP $\delta$ puncta that overlapped with GAD67 were also observed on axons that were not in contact with dendrites, suggesting an axonal localization (Fig. 2D,E). Thus, endogenous PTP $\delta$ is also specifically localized to inhibitory synaptic sites, consistent with the distribution pattern of recombinant $\mathrm{PTP} \delta$ proteins (Takahashi et al., 2012). Endogenous PTP $\sigma$ puncta showed a greater degree of colocalization with VGLUT1 than with vesicular GABA transporter (VGAT), as previously reported (Takahashi et al., 2011) (Fig. 2F,G).

We then asked whether KD of PTP $\sigma$ or PTP $\delta$ changed the expression of specific proteins known to be functionally linked to 


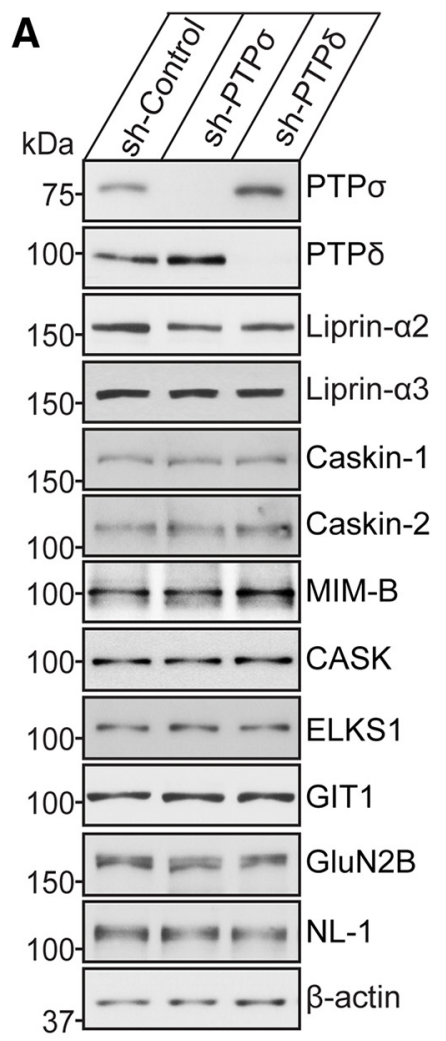

\section{B Quantitation for panel A}

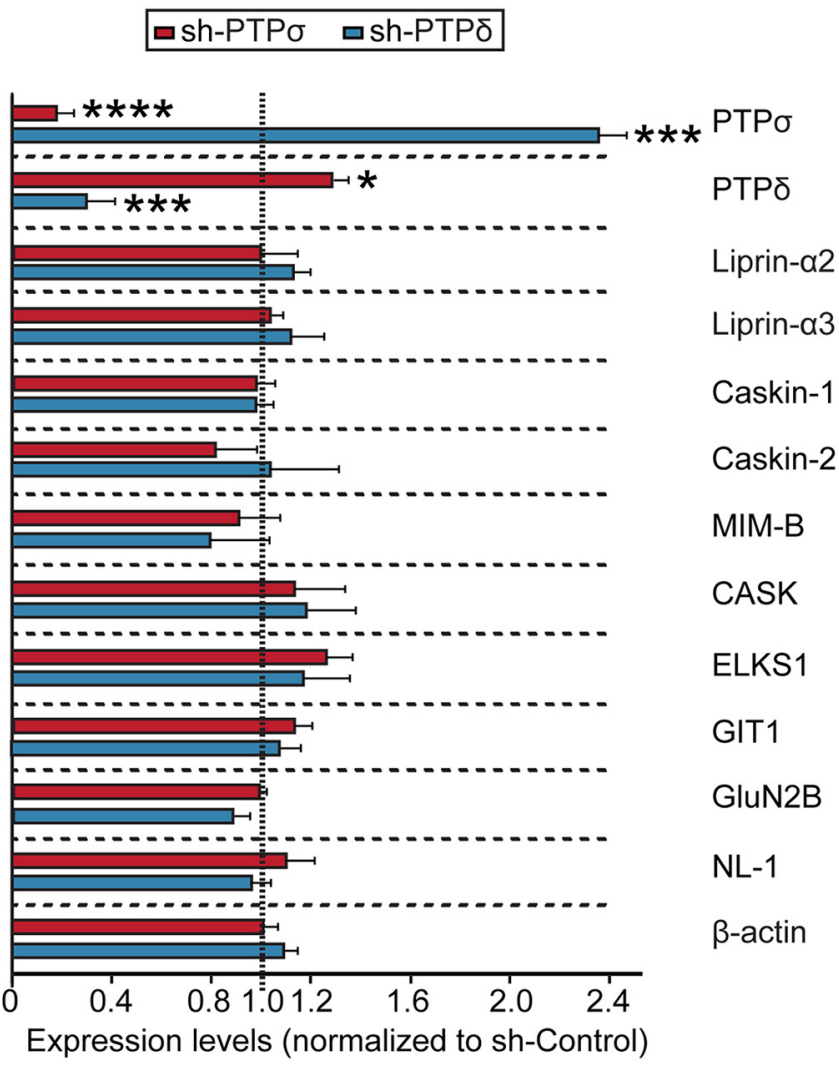

Figure 3. Effects of PTP $\sigma$ and PTP $\delta$ KD on the levels of selected synaptic proteins in cultured neurons. $A, B$, Representative semiquantitative immunoblot images $(\boldsymbol{A})$ and quantitative summary $(\boldsymbol{B})$ of the expression levels of the indicated synaptic proteins in cultured cortical neurons infected with sh-Control, sh-PTP $\sigma$, or sh-PTP $\delta$ lentiviruses. $\beta$-Actin was used as a loading control, and protein levels were expressed relative to those in the sh-Control group. Data are mean \pm SEM. Parametric $t$ test: ${ }^{*} p<0.05 ;{ }^{* * *} p<0.001 ;{ }^{* * * *} p<0.0001 ; n=$ number of samples as follows: PTP $\sigma, n=4 ;$ PTP $\delta, n=4$; liprin- $\alpha 2, n=3$; liprin- $\alpha 3, n=3$; Caskin-1, $n=4$; Caskin-2, $n=3 ;$ MIM-B, $n=3$; CASK, $n=3$; ELKS1, $n=3 ;$ GIT1, $n=3 ;$ GluN2B, $n=3 ;$ NL-1, $n=3$; and $\beta$-actin, $n=6$. $p$ values for PTP $\sigma$ blots: sh-Control vs sh-PTP $\sigma, p<0.0001$; and sh-Control vs sh-PTP $\delta, p=0.0003$. p values for PTP $\delta$ blots: sh-Control vs sh-PTP $\sigma, p=0.0108$; and sh-Control vs sh-PTP $\delta$, $p=0.0005$. $p$ values for liprin- $\alpha 2$ blots: sh-Control vs sh-PTP $\sigma, p=0.6963$; and sh-Control vs sh-PTP $\delta, p=0.4269$. $p$ values for liprin- $\alpha 3$ blots: sh-Control vs sh-PTP $\sigma, p=0.524 ;$ and sh-Control vs sh-PTP $\delta, p=0.437$. $p$ values for Caskin- 1 blots: sh-Control vs sh-PTP $\sigma, p=0.643$; and sh-Control vs sh-PTP $\delta, p=0.6568$. $p$ values for Caskin-2 blots: sh-Control vs sh-PTP $\sigma, p=0.2875$; and sh-Control vs sh-PTP $\delta, p=0.8973$. $p$ values for MIM-B blots: sh-Control vs sh-PTP $\sigma, p=0.5808$; and sh-Control vs sh-PTP $\delta, p=0.4229$. $p$ values for CASK blots: sh-Control vs sh-PTP $\sigma, p=$ 0.5549; and sh-Control vs sh-PTP $\delta, p=0.4523$. $p$ values for ELKS1 blots: sh-Control vs sh-PTP $\sigma, p=0.0756$; and sh-Control vs sh-PTP $\delta, p=0.4072$. $p$ values for GIT1 blots: sh-Control vs sh-PTP $\sigma$, $p=0.0878$; and sh-Control vs sh-PTP $\delta, p=0.3826$. $p$ values for GluN2B blots: sh-Control vs sh-PTP $\sigma, p=0.4656$; and sh-Control vs sh-PTP $\delta, p=0.0672$. $p$ values for NL-1 blots: sh-Control vs sh-PTP $\sigma, p=0.4262$; and sh-Control vs sh-PTP $\delta, p=0.5466$. $p$ values for $\beta$-actin blots: sh-Control vs sh-PTP $\sigma, p=0.662$; and sh-Control vs sh-PTP $\delta, p=0.9238$.

LAR-RPTPs. To test this, we performed semiquantitative immunoblot analyses of 9 selected synaptic proteins (liprin- $\alpha 2$, liprin$\alpha 3$, Caskin-1, Caskin-2, MIM-B, CASK, ELKS1, GIT1, and GluN2B) in cultured cortical neurons after shRNA-mediated KD of PTP $\sigma$ or PTP $\delta$. We found no alterations in any of these synaptic proteins (Fig. $3 A, B$ ). Interestingly, however, PTP $\sigma$ KD decreased the levels of PTP $\sigma$ mRNA by $\sim 75 \%$ (Fig. $1 C$ ) and PTP $\sigma$ protein by $\sim 84 \%$ (Fig. $3 A, B$ ), and concurrently increased the levels of PTP $\delta$ proteins by $127 \%$, without affecting PTP $\delta$ mRNA levels (Figs. $1 C, 3 A, B$ ). Similarly, PTP $\delta \mathrm{KD}$ reduced the levels of PTP $\delta$ mRNA by $\sim 80 \%$ (Fig. $1 C$ ) and PTP $\delta$ protein by $\sim 70 \%$ (Fig. $3 A, B$ ) while dramatically upregulating PTP $\sigma$ protein levels by $235 \%$ (Fig. $3 A, B$ ). These results indicate that loss of PTP $\sigma$ may activate a compensatory mechanism that upregulates PTP $\delta$, and vice versa.

We next tested the effect of PTP $\sigma$ or PTP $\delta$ KD on the density of synapses formed between neurons (Fig. $4 A-E$ ) and synaptic transmission in cultured hippocampal neurons (Fig. $4 F-K$ ). In line with our previous observation, immunocytochemical analyses showed that PTP $\sigma$ KD specifically decreased the degree of excitatory, but not inhibitory, synapse density, an effect that was completely rescued by reexpression of full-length WT PTP $\sigma$ (Ko et al., 2015b) (Fig. 4A-E). Conversely, PTP $\delta$ KD specifically reduced the degree of inhibitory, but not excitatory, synapse density, an effect that was similarly rescued by reexpression of fulllength WT PTP $\delta$ (Fig. 4A-E). To corroborate the anatomical effects of PTP $\sigma$ or PTP $\delta$ KD, we next infected cultured hippocampal neurons with lentiviruses expressing sh-PTP $\sigma$, shPTP $\delta$, sh-LAR, or sh-Control at DIV3, and recorded mEPSCs or mIPSCs at DIV14-DIV15. PTP $\sigma$ KD significantly decreased the frequency and amplitude of mEPSCs, consistent with our previous report (Ko et al., 2015b), whereas PTP $\delta$ KD specifically decreased the frequency and amplitude of mIPSCs (Fig. 4F-K). Collectively, these immunocytochemical and electrophysiological analyses reveal that $\operatorname{PTP} \sigma$ and $\operatorname{PTP} \delta$ are required for excitatory and inhibitory synapse development, respectively, in cultured hippocampal neurons.

\section{The extracellular region of PTP $\sigma$ is required for} presynaptic assembly

To determine whether specific PTP $\sigma$ splice variants containing $\mathrm{MeA}$ or $\mathrm{MeB}$ inserts responsible for Ig domain binding are 


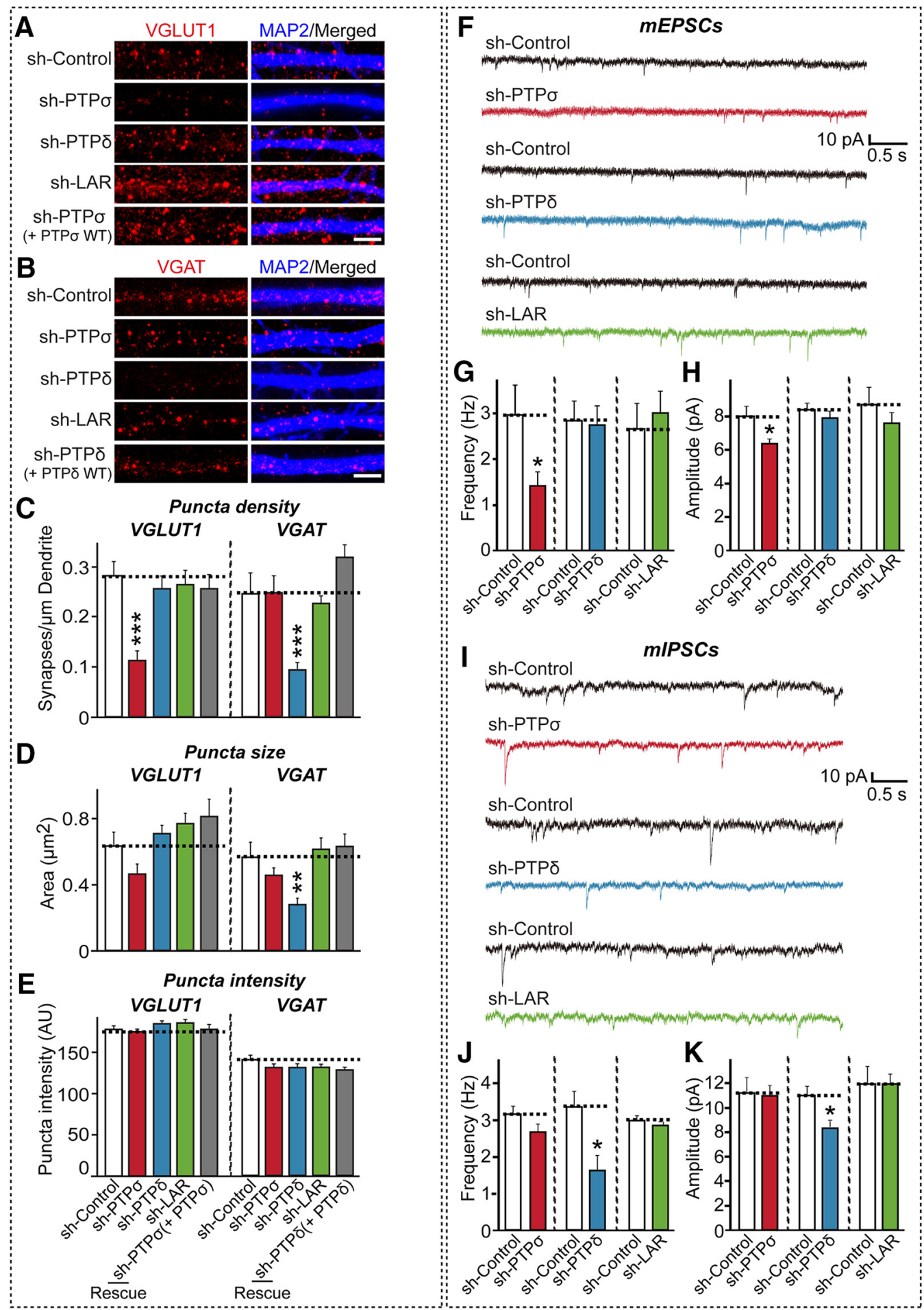

Figure 4. Effects of PTP $\sigma$ and PTP $\delta$ KD on synapse density and synaptic transmission in cultured neurons. $A, B$, Cultured hippocampal neurons were infected with lentiviruses expressing sh-Control, sh-LAR, sh-PTP $\sigma$, or sh-PTP $\delta$, or were coinfected with lentiviruses expressing sh-PTP $\sigma$ or sh-PTP $\delta$ together with shRNA-resistant full-length PTP $\sigma(+$ PTP $\sigma$ WT) or sh-PTP $\delta$ ( + PTP $\delta$ WT) at DIV4, and analyzed at DIV14 by double-immunofluorescence detection of MAP2 and VGLUT1 ( $\boldsymbol{A})$ or VGAT (B). Scale bar (all images), $10 \mu \mathrm{m}$. $\boldsymbol{C}-\boldsymbol{E}$, Summary graphs of $\boldsymbol{A}, \boldsymbol{B}$. Synaptic puncta density $(\boldsymbol{C}$, synaptic puncta size $(\boldsymbol{D})$, and synaptic puncta intensity $(\boldsymbol{E})$ were measured using VGLUT1 and VGAT as excitatory and inhibitory synaptic markers, respectively. Two or three dendrites per infected neurons were analyzed and group-averaged. Data are mean \pm SEM. Mann-Whitney $U$ test: ${ }^{* *} p<0.01 ;{ }^{* * *} p<0.001 . n=$ number of neurons as follows: (E) sh-Control, $n=17$; sh-PTP $\sigma, n=16$; sh-PTP $\delta, n=15$; sh-LAR, $n=15$; and sh-PTP $\sigma+$ PTP $\sigma$ WT, $n=13$. F, sh-Control, $n=13$; sh-PTP $\sigma, n=14$; sh-PTP $\delta, n=14 ;$ sh-LAR, $n=14$; and sh-PTP $\delta+$ PTP $\delta$ WT, $n=$ 14. $p$ values for VGLUT1 puncta density: sh-Control vs sh-PTP $\sigma, p<0.001$; sh-Control vs sh-PTP $\delta, p=0.3322$; sh-Control vs sh-LAR, $p=0.3765$; and sh-Control vs sh-PTP $\sigma(+\mathrm{PTP} \sigma), p=0.8210$. $p$ values for VGAT puncta density: sh-Control vs sh-PTP $\sigma, p=0.9781$; sh-Control vs sh-PTP $\delta, p<0.001$; sh-Control vs sh-LAR, $p=0.1958$; and sh-Control vs sh-PTP $\delta$ (+PTP $\delta$ ), $p=0.8124$. $p$ values for VGLUT1 puncta size: sh-Control vs sh-PTP $\sigma, p=0.0820$; sh-Control vs sh-PTP $\delta, p>0.9999$; sh-Control vs sh-LAR, $p=0.9999$; and sh-Control vs sh-PTP $\sigma(+\mathrm{PTP} \sigma), p=0.9212 . p$ values for VGAT puncta size: sh-Control vs sh-PTP $\sigma, p=0.1248$; sh-Control vs sh-PTP $\delta, p<0.01$; sh-Control vs sh-LAR, $p>0.9999$; and sh-Control vs sh-PTP $\delta$ ( + PTP $\delta$ ), $p=0.8790$. $p$ values for VGLUT1 puncta intensity: sh-Control vs sh-PTP $\sigma, p=0.7176$; sh-Control vs sh-PTP $\delta, p=0.8392$; sh-Control vs sh-LAR, $p=0.9115$; and sh-Control vs sh-PTP $\sigma(+$ PTP $\sigma), p=0.7938$. $p$ values for VGAT puncta intensity: sh-Control vs sh-PTP $\sigma, p=0.2190$; sh-Control vs sh-PTP $\delta, p=0.4771$; sh-Control vs sh-LAR, $p=0.3198$; and sh-Control vs sh-PTP $\delta$ ( + PTP $\delta$ ), $p=0.6787$. F, $I$, Representative traces of mEPSCS $(\boldsymbol{F})$ and mIPSCS $(\boldsymbol{I})$ in neurons infected with control, shPTP $\sigma$, sh-PTP $\delta$, or sh-LAR lentiviruses. Neurons were infected with lentiviruses at (Figure legend continues.) 
involved in the maintenance of excitatory synapse development in neurons, we infected PTP $\sigma$-deficient neurons with lentiviruses expressing each of four different PTP $\sigma$ splice variants $\left(\mathrm{PTP} \sigma^{\mathrm{MeA}+\mathrm{MeB}+}, \mathrm{PTP} \sigma^{\mathrm{MeA}-\mathrm{MeB}+}, \mathrm{PTP} \sigma^{\mathrm{MeA}+\mathrm{MeB}-}\right.$, or $\left.\mathrm{PTP} \sigma^{\mathrm{MeA}-\mathrm{MeB}-}\right)$ and analyzed excitatory synapse density, as determined by VGLUT1 staining (Fig. 5). We found that expression of each of the four PTP $\sigma$ splice variants rescued the deficit in excitatory synapse density observed in PTP $\sigma$-deficient neurons, indicating that PTP $\sigma$ is able to mediate presynaptic assembly independent of binding partners that interact through its Ig domains.

Presynaptic neurexins are required for induction of heterologous synapse formation by their postsynaptic ligands, neuroligin-1 or leucine-rich repeat transmembrane protein (LRRTM2), but only the extracellular regions of neurexins are required for neurexinmediated signal transduction in presynaptic neurons (Gokce and Südhof, 2013). To examine whether presynaptic LAR-RPTPs mediate signal transduction in presynaptic neurons, we first generated lentiviral expression vectors for a variety of PTP $\sigma$ deletion variants and point mutants containing a HA epitope tag, which we had introduced into most PTP $\sigma$ rescue constructs to monitor expression level and surface transport (Fig. 6). Because we primarily used Slitrk1 (see Figs. 7-9) or TrkC (see Fig. 10) to induce heterologous synapse formation in the current study, we used a $\mathrm{PTP} \sigma$ splice variant containing both the MeA and MeB inserts $\left(\mathrm{PTP} \sigma^{\mathrm{MeA}+\mathrm{MeB}+}\right)$ to generate various PTP $\sigma$ rescue vectors (Figs. 6-10, 12) (Coles et al., 2014; Um et al., 2014b). All PTP $\sigma$ variants exhibited the expected molecular weights in immunoblot analyses (Fig. 6B), and, with the exception of a glycosylphosphatidylinositol (GPI)-anchored construct, was effectively targeted to the cell surface following expression in HEK293T cells (Fig. 6C) and bound well to known ligands (NGL-3 or GPC-4), as expected (Fig. 6D; see Fig. 8B).

Using this PTP $\sigma$ "toolkit," we performed heterologous synapse-formation assays using HEK293T cells expressing either Slitrk1 or TrkC, and cultured neurons infected with lentiviruses expressing the indicated PTP $\sigma$ constructs. We found that heterologous synapse formation induced by Slitrk1 or TrkC was impaired in PTP $\sigma$-deficient neurons, a defect that was completely rescued by reintroduction of PTP $\sigma \mathrm{WT}$ (+WT) (see Figs. 7-10). Reexpression of PTP $\sigma$ variants lacking Ig domains $(+\Delta \mathrm{Ig})$ or the entire extracellular region $(+\Delta$ Ecto $)$ failed to rescue the impaired heterologous synapse formation, in accord with the requirement for direct interactions of PTP $\sigma$ with Slitrk1 or TrkC through Ig domains (Fig. 7, 10C,D) (Um and Ko, 2013). In contrast, reexpression of a heparan sulfate (HS)-binding-defective PTP $\sigma$ mutant (+ AAAA) or one in which the first two fibronectin Type III

\footnotetext{
(Figure legend continued.) DIV4, and electrophysiological recordings were obtained at DIV14-16. $\boldsymbol{G}, \boldsymbol{H}$, Summary graphs of the frequencies $(\boldsymbol{G})$ and amplitudes $(\boldsymbol{H})$ of mEPSCs in neurons infected with control, shPTP $\sigma$, sh-PTP $\delta$, or sh-LAR lentiviruses. Data are mean \pm SEM. ${ }^{*} p<0.05$ (Student's test). $n=$ number of neurons as follows: sh-Control 1, $n=16$; sh-PTP $\sigma$, $n=21$; sh-Control 2, $n=13$; sh-PTP $\delta, n=30$; sh-Control 3, $n=11$; and sh-LAR, $n=16$. $p$ values for mEPSC frequency: sh-Control 1 vs sh-PTP $\sigma, p<0.05$; sh-Control 2 vs sh-PTP $\delta, p=$ 0.4237; and sh-Control 3 vs sh-LAR, $p=0.5211$. $p$ values for mEPSC amplitude: sh-Control 1 vs sh-PTP $\sigma, p<0.05$; sh-Control 2 vs sh-PTP $\delta, p=0.8921$; and sh-Control 3 vs sh-LAR, $p=$ 0.9783. J, $\boldsymbol{K}$, Same as $\boldsymbol{G}, \boldsymbol{H}$, except that mIPSCs were measured. Data are mean \pm SEM. ${ }^{*} p<$ 0.05 (Student's $t$ test). $n=$ number of neurons as follows: sh-Control 1, $n=16$; sh-PTP $\sigma, n=$ 23; sh-Control 2, $n=11$; sh-PTP $\delta, n=20$; sh-Control $3, n=16$; and sh-LAR, $n=23$. $p$ values for mIPSC frequency: sh-Control 1 vs sh-PTP $\sigma, p=0.3576$; sh-Control 2 vs sh-PTP $\delta, p<0.05$; and sh-Control 3 vs sh-LAR, $p=0.6183$. $p$ values for mIPSC amplitude: sh-Control 1 vs sh-PTP $\sigma$, $p=0.7120$; sh-Control 2 vs sh-PTP $\delta, p<0.05$; and sh-Control 3 vs sh-LAR, $p=0.8217$.
}

$\leftarrow$ repeats were deleted $(+\Delta \mathrm{FN} 1-2)$ effectively rescued the impaired heterologous synapse formation (see Figs. 7, 10C,D). Moreover, reexpression of a proteolytically uncleavable $\mathrm{PTP} \sigma$ mutant (+R781A) (Dunah et al., 2005) fully rescued the defective synaptogenic activity, indicating that cleavage by furin/subtilisin-like endoproteases is not necessary for the heterologous synapse formation activity (see Figs. 7, 10C,D). This contrasts with the requirement of endoprotease-mediated cleavage of PTP $\sigma$ for the maintenance of excitatory neuronal synapse structures (Dunah et al., 2005).

We also examined whether the extracellular region of PTP $\sigma$ is required to maintain excitatory synapse density in neuronal dendrites (Fig. 7). Extracellular interactions through PTP $\sigma$ Igdomains and HS-binding residues, but not FN1-2, were required for regulation of excitatory synapse development in neuronal synapses (Fig. 7) (Coles et al., 2011; Ko et al., 2015b). On the basis of these data, we conclude that PTP $\sigma$ organizes heterologous synapse formation and neuronal synapse development through mechanisms involving the extracellular region.

\section{PTPo-mediated maintenance of excitatory synapse development in cultured hippocampal neurons requires interaction with Slitrks, but not with TrkC or GPC-4}

The observation that extracellular interactions with various ligands are necessary for the excitatory synapse-promoting activity of PTP $\sigma$ in neurons prompted us to test which PTP $\sigma$ ligands are most important. Addressing this question requires information about the crystal structure of PTP $\sigma$ complexes with ligands to pinpoint critical residues involved in the interactions with specific ligands. Among the known PTP $\sigma$ ligands, structural information is currently available only for Slitrk1 and TrkC (Coles et al., 2014; Um et al., 2014a). Based on the published crystal structures of Slitrk1-PTP $\delta$ and TrkC-PTP $\sigma$ complexes, we designed the following three point mutants: R97A/R100A, which specifically disrupts binding to TrkC, without affecting Slitrk1 binding; Y233S, which blocks binding to both Slitrk1 and TrkC; and R235D, which specifically disrupts bindings to Slitrk1, but not to TrkC (Fig. 8). All three PTP $\sigma$ mutants were expressed well on the surface of transfected HEK293T cells and bound recombinant NGL-3-Fc proteins (Figs. 6C, 8B). PTP $\sigma$ R97A/R100A, but not $\mathrm{PTP} \sigma \mathrm{Y} 233 \mathrm{~S}$ or PTP $\sigma$ R235D, showed impaired binding to recombinant GPC-4-Fc proteins (Fig. $8 B$ ), partly because of the fact that R97 and R100 residues of PTP $\sigma$ also contribute to glycosaminoglycan binding (Aricescu et al., 2002; Coles et al., 2011). We next performed heterologous synapse-formation assays using HEK293T cells expressing either Slitrk1 (Fig. 8C,D) or TrkC (see Fig. 10), and cultured neurons infected with lentiviruses expressing sh-Control or sh-PTP $\sigma$, or coexpressing sh-PTP $\sigma$ and the indicated PTP $\sigma$ constructs (WT, R97/100A, Y233S, or R235D). We found that impaired Slitrk1-mediated heterologous synapse formation with PTP $\sigma$-deficient neurons was completely rescued by reexpression of PTP $\sigma$ WT $(+\mathrm{WT})$ or PTP $\sigma$ R97A/R100A $(+\mathrm{R} 97 / 100 \mathrm{~A})$ in neurons (Fig. 8C,D). Reexpression of PTP $\sigma$ Y233S (+Y233S) or PTP $\sigma$ R235D (+R235D) failed to rescue the impaired heterologous synapse formation elicited by Slitrk1, consistent with our previous report that these two PTP $\sigma$ mutations abolish interactions with Slitrks (Fig. 8C,D) (Um et al., 2014a). Similarly, impaired TrkC-mediated heterologous synapse formation with PTP $\sigma$-deficient neurons was selectively rescued by reexpression of PTP $\sigma$ WT $(+\mathrm{WT})$ or PTP $\sigma$ R235D (+R235D) (see Fig. 10A,B).

Using these characterized PTP $\sigma$ point mutants, we then asked which PTP $\sigma$-interacting extracellular proteins primarily operate to- 
A

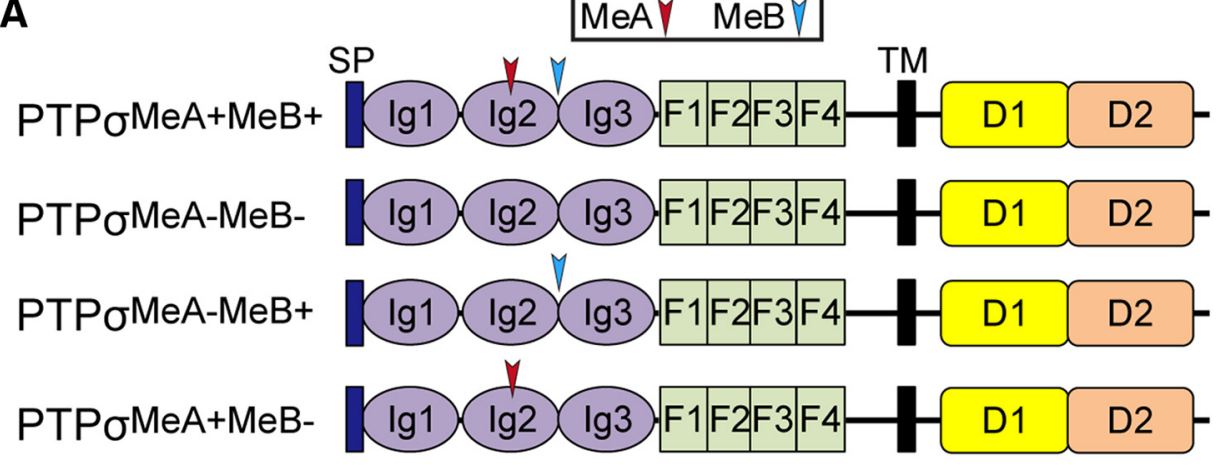

B

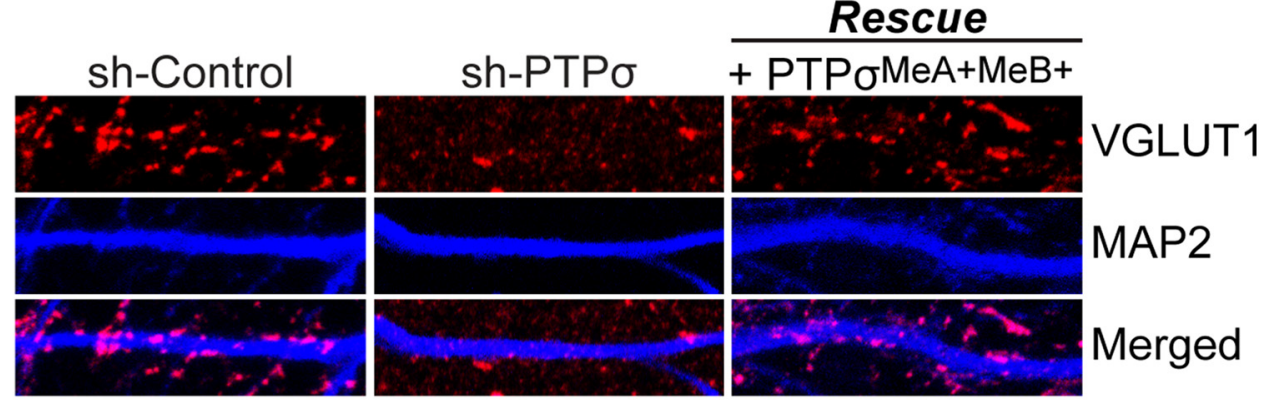

Rescue

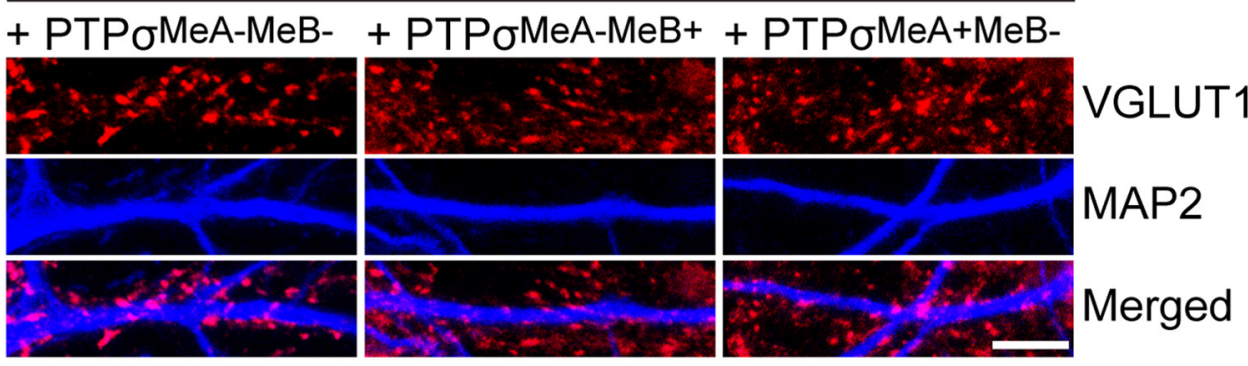

C

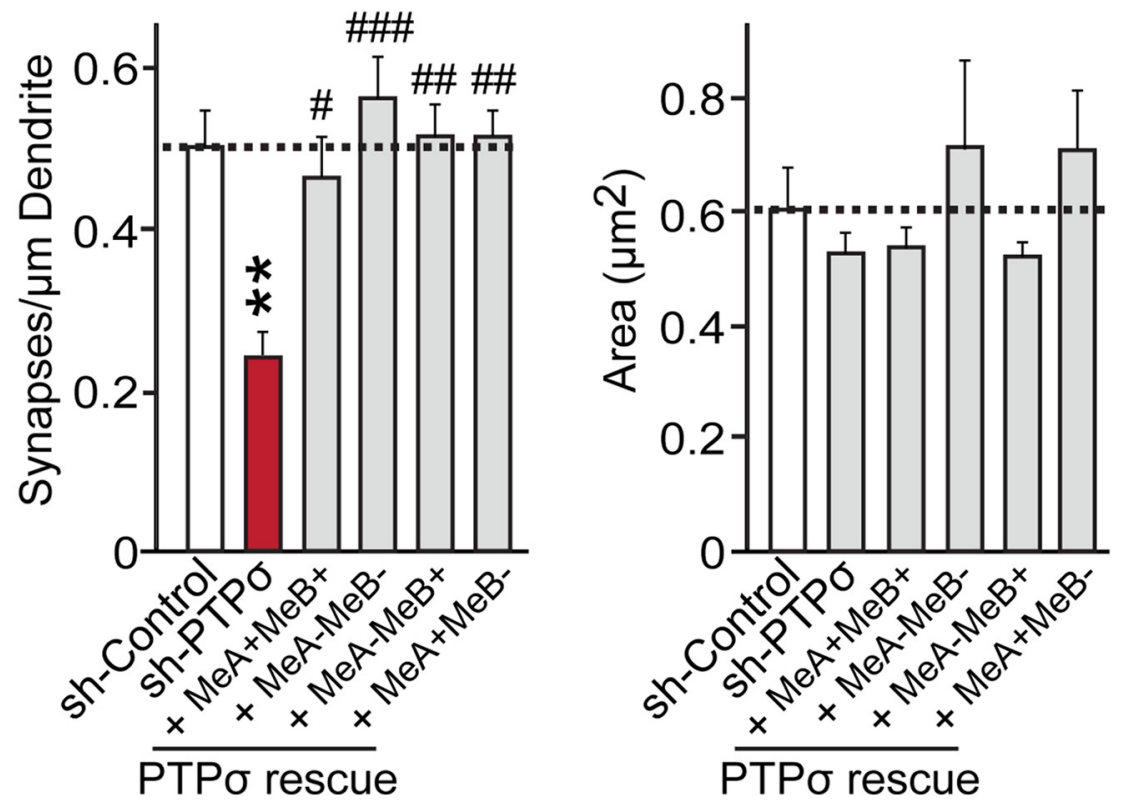

Figure 5. Effect of PTP $\sigma$ alternative splicing on excitatory synapse development in cultured neurons. $A$, Schematic diagrams of PTP $\sigma$ alternative splice variants used in the current study. D1, first catalytic domain of LAR-RPTPS; D2, second catalytic domain of LAR-RPTPs; F, fibronectin Type III repeat; Ig, Ig domain; MeA, mini-exon A; MeB; mini-exon B; SP, Ig $<B$ signal peptide contained in the pDisplay vector (Invitrogen); TM, transmembrane region. $\boldsymbol{B}$, Representative images from cultured hippocampal neurons infected at DIV4 with lentiviruses expressing sh-Control, sh-PTP $\sigma$, or coinfected with lentiviruses expressing sh-PTP $\sigma$ and the human PTP $\sigma$ alternatively spliced variant rescue viruses, $\mathrm{PTP} \sigma^{\mathrm{MeA}+\mathrm{MeB}+}, \mathrm{PTP} \sigma^{\mathrm{MeA}-\mathrm{MeB}-}{ }^{,}, \mathrm{PTP} \sigma^{\mathrm{MeA}-\mathrm{MeB}+}$, or PTP $\sigma^{\mathrm{MeA}+\mathrm{MeB}-}$, and analyzed at DIV14 by double-immunofluorescence detection of MAP2 (blue) and the excitatory synaptic marker VGLUT1 (red). Scale bar (all images), $10 \mu \mathrm{m}$. C, Summary graphs of the effects of PTP $\sigma$ splice variants in neurons on puncta density (left) and puncta size (right), measured using VGLUT1 as an excitatory synaptic marker. Two or three dendrites per (Figure legend continues.) 
gether with PTP $\sigma$ in the maintenance of excitatory synapse development. Strikingly, the interaction of PTP $\sigma$ with Slitrks rather than that with TrkC or GPC-4 appeared to be more critical, although the significance of TrkC or GPC-4 in PTP $\sigma$ function cannot be discounted because the HS-binding property of PTP $\sigma$ is also required for PTP $\sigma$-mediated excitatory synaptic transmission (Fig. $8 E, F$ ) (Ko et al., 2015b). In addition, it should be noted that contributions of other extracellular $\mathrm{PTP} \sigma$-interacting proteins involved in PTP $\sigma$ function, such as NGL-3 and SALMs, could not be assessed in the current study. Collectively, our data suggest that a set of specific extracellular ligand(s) is used for PTP $\sigma$-mediated excitatory synapse development.

\section{Direct interaction of PTP $\sigma$ with liprin- $\alpha$ through the PTP $\sigma$ D2 domain is required for presynaptic assembly}

As expected given the requirement for cytoplasmic regions of the PTP $\sigma$ protein in mediating heterologous synapse formation (Um and Ko, 2013), reintroduction of a PTP $\sigma$ deletion variant lacking the entire cytoplasmic region into PTP $\sigma$-deficient neurons also failed to rescue the impaired Slitrk1- and TrkC-mediated heterologous synapse formation activity, unlike the heterologous synapse formation activity induced by NL-1 (Figs. 9, 10 E, F) (Gokce and Südhof, 2013). To further dissect the requirement of PTP $\sigma$ cytoplasmic sequences in mediating presynaptic assembly, we performed heterologous synapse-formation assays using HEK293T cells expressing either Slitrk1 or TrkC and cultured neurons infected with lentiviruses expressing the indicated PTP $\sigma$ deletion constructs and point mutants. We found that the impaired heterologous synapse formation activity observed in $\mathrm{PTP} \sigma$ deficient neurons was not rescued by lentiviral expression of a PTP $\sigma$ deletion variant that lacked the entire cytoplasmic region $(+\Delta$ Cyto $)$ or the $\mathrm{D} 2$ domain $(+\Delta \mathrm{D} 2)$, suggesting that interactions of $\mathrm{PTP} \sigma$ with intracellular proteins are necessary for excitatory presynaptic assembly (Fig. 9B, $C, 10 E, F$ ). We also examined whether the intracellular region of PTP $\sigma$ is required to maintain excitatory synapse density in neuronal dendrites (Fig. 9D-F). We found that PTP $\sigma$ D2 domainmediated protein interactions and tyrosine phosphorylation signaling pathways were required to regulate the development of excitatory synapses (Fig. 9).

To extend previous in vitro results identifying diverse LARRPTP-interacting proteins (Um and Ko, 2013), we sought further support for the requirement of the $\mathrm{PTP} \sigma \mathrm{D} 2$ domain in presynaptic assembly in vivo by, first, conducting GST pull-down assays in mouse brain synaptosomal fractions using recombinant PTP $\sigma$

\section{$\leftarrow$}

(Figure legend continued.) infected neuron were analyzed and group-averaged. Data are mean \pm SEM. ANOVA with a nonparametric Kruskal-Wallis test: ${ }^{* *} p<0.01 ;{ }^{\#} p<0.05 ;{ }^{\# \#} p<$ $0.01 ; \# \#<0.001 . n=$ number of neurons as follows: sh-Control/VGLUT1, $n=14$; sh-PTP $\sigma /$ VGLUT1, $n=15 ;$ PTP $\sigma^{\text {MeA }+ \text { MeB }+}$ rescue/VGLUT1, $n=15 ;$ PTP $\sigma^{\mathrm{MeA}-\mathrm{MeB}-}$ rescue/VGLUT1, $n=13 ; \mathrm{PTP} \sigma^{\mathrm{MeA}-\mathrm{MeB}+}$ rescue/VGLUT1, $n=15$; and PTP $\sigma^{\mathrm{MeA}+\mathrm{MeB}-}$ rescue/VGLUT1, $n=$ 15. $p$ values for puncta density: sh-Control vs sh-PTP $\sigma, p=0.0065$; sh-Control vs PTP $\sigma^{\mathrm{MeA}+\mathrm{MeB}+}$ rescue, $p>0.9999$; sh-Control vs PTP $\sigma^{\mathrm{MeA}-\mathrm{MeB}-}$ rescue, $p>0.9999$; shControl vs PTP $\sigma^{\mathrm{MeA}-\mathrm{MeB}+}$ rescue, $p>0.9999$; sh-Control vs PTP $\sigma^{\mathrm{MeA}+\mathrm{MeB}}$ rescue, $p>$ $0.9999 ;$ sh-PTP $\sigma$ vs PTP $\sigma^{\mathrm{MeA}+\mathrm{MeB}+}$ rescue, $p=0.0304$; sh-PTP $\sigma$ vs PTP $\sigma^{\mathrm{MeA}-\mathrm{MeB}-}$ rescue, $p=0.0002 ;$ sh-PTP $\sigma$ vs PTP $\sigma^{\mathrm{MeA}-\mathrm{MeB}+}$ rescue, $p=0.0015$; and sh-PTP $\sigma$ vs PTP $\sigma^{\mathrm{MeA}+\mathrm{MeB}}$ rescue, $p=0.002$. $p$ values for puncta size: sh-Control vs sh-PTP $\sigma, p>0.9999$; sh-Control vs PTP $\sigma^{\mathrm{MeA}+\mathrm{MeB}+}$ rescue, $p>0.9999$; sh-Control vs PTP $\sigma^{\mathrm{MeA}-\mathrm{MeB}-}$ rescue, $p>0.9999$; shControl vs PTP $\sigma^{\mathrm{MeA}-\mathrm{MeB}+}$ rescue, $p>0.9999$; sh-Control vs PTP $\sigma^{\mathrm{MeA}+\mathrm{MeB}}$ rescue, $p>$ 0.9999 ; sh-PTP $\sigma$ vs PTP $\sigma^{\mathrm{MeA}+\mathrm{MeB}+}$ rescue, $p>0.9999$; sh-PTP $\sigma$ vs PTP $\sigma^{\text {MeA }- \text { MeB }-}$ rescue, $p>0.9999 ;$ sh-PTP $\sigma$ vs PTP $\sigma^{\mathrm{MeA}-\mathrm{MeB}+}$ rescue, $p>0.9999$; and sh-PTP $\sigma$ vs PTP $\sigma^{\mathrm{MeA}+\mathrm{MeB}}$ rescue, $p>0.9999$.
D2 and PTP $\delta$ D2 proteins. Our mass spectroscopy analyses identified a subset of presynaptic proteins that had previously been reported to directly interact with LAR-RPTPs, including liprin$\alpha 3$, ELKS1, CASK, and Caskin-1 (Fig. 11-1, available at https:// doi.org/10.1523/JNEUROSCI.0672-18.2018.f11-1). The top priority among numerous putative candidate peptides was liprin- $\alpha$ family proteins because of the established functions of invertebrate orthologs in the CNS and their multiple interactions with other presynaptic active zone proteins and scaffolds, including RIM1, CASK, mSYD-1, GIT1 (G-protein coupled receptor interacting protein 1), and GRIPs (glutamate receptor-interacting proteins) (Spangler and Hoogenraad, 2007; Südhof, 2012; Han et al., 2016). Among four liprin- $\alpha$ isoforms, we focused on liprin- $\alpha 2$ and liprin- $\alpha 3$ because they are major liprin- $\alpha$ isoforms in the brain (Zürner et al., 2011). After screening liprin- $\alpha 2$ and liprin- $\alpha 3$ shRNAs for the most effective sequences, we constructed an sh-liprin- $\alpha 2 / \alpha 3$-expressing lentiviral RNAi vector (L-309) (Pang et al., 2010) to simultaneously knock down both liprin- $\alpha 2$ and liprin- $\alpha 3$ (Fig. 11E). We then performed heterologous synapse-formation assays in cultured neurons infected with lentiviruses expressing sh-liprin- $\alpha 2 / \alpha 3$. We found that $\mathrm{KD}$ of liprin- $\alpha 2$ and liprin- $\alpha 3$ impaired the heterologous synapse formation activity induced by Slitrk6 or NL-2 (Fig. 11A,B). These results reinforce the idea that the D2 domain of LAR-RPTPs acts an anchoring point for mediating intracellular signaling downstream of LAR-RPTPs for presynaptic assembly, notably through liprin- $\alpha$ and its multiprotein complexes, and that both LARRPTPs and neurexins orchestrate heterologous synapse formation through converging signaling pathways involving liprin- $\alpha 2$ and liprin- $\alpha 3$.

\section{Specific PTP $\sigma$ substrates are required for PTP $\sigma$-mediated presynaptic assembly}

The intracellular region of PTP $\sigma$ harboring its phosphatase activity partly appears to be critical for the presynaptic assembly process, as shown by the ability of lentiviral-mediated expression of $\mathrm{PTP} \sigma$ C1157S or PTP $\sigma$ D1125A, catalytically inactive mutants of $\mathrm{PTP} \sigma$, to partially rescue the impaired heterologous synapse-formation activity in $\mathrm{PTP} \sigma$-deficient neurons (Figs. 9, 10) (Johnson and Van Vactor, 2003). Thus, we examined whether a subset of PTP $\sigma$ substrates are involved in PTP $\sigma$-mediated presynaptic assembly. The currently known LAR-RPTP substrates include N-cadherin, $\beta$-catenin, Abelson kinase (Abl), Enabled (Ena), Trio, and p250GAP (Han et al., 2016). Of these putative substrates, three proteins, N-cadherin, $\beta$-catenin, and p250GAP, for which highly efficient shRNA sequences were available in our laboratory, were selected for further study (Fig. 11E). Remarkably, KD of p250GAP or N-cadherin, but not KD of $\beta$-catenin, abolished the heterologous synapseformation activity induced by Slitrk6 (Fig. 11C,D), suggesting that a specific set of substrate proteins is involved in recruiting the presynaptic machinery involved in LAR-RPTP-mediated heterologous synapse formation. Together with our previous results, these data suggest that the recruitment of presynaptic machinery into nascent synaptic sites for heterologous synapse formation is accompanied by the concentration of intracellular scaffolds and substrates that directly or indirectly associate with PTP $\sigma$ through the $\mathrm{D} 2$ domain.

\section{The PTP $\sigma$ D2 domain alone is insufficient to drive presynaptic assembly at excitatory synapses}

Although our results unambiguously demonstrate a structural prerequisite for the action of LAR-RPTP family proteins in pre- 

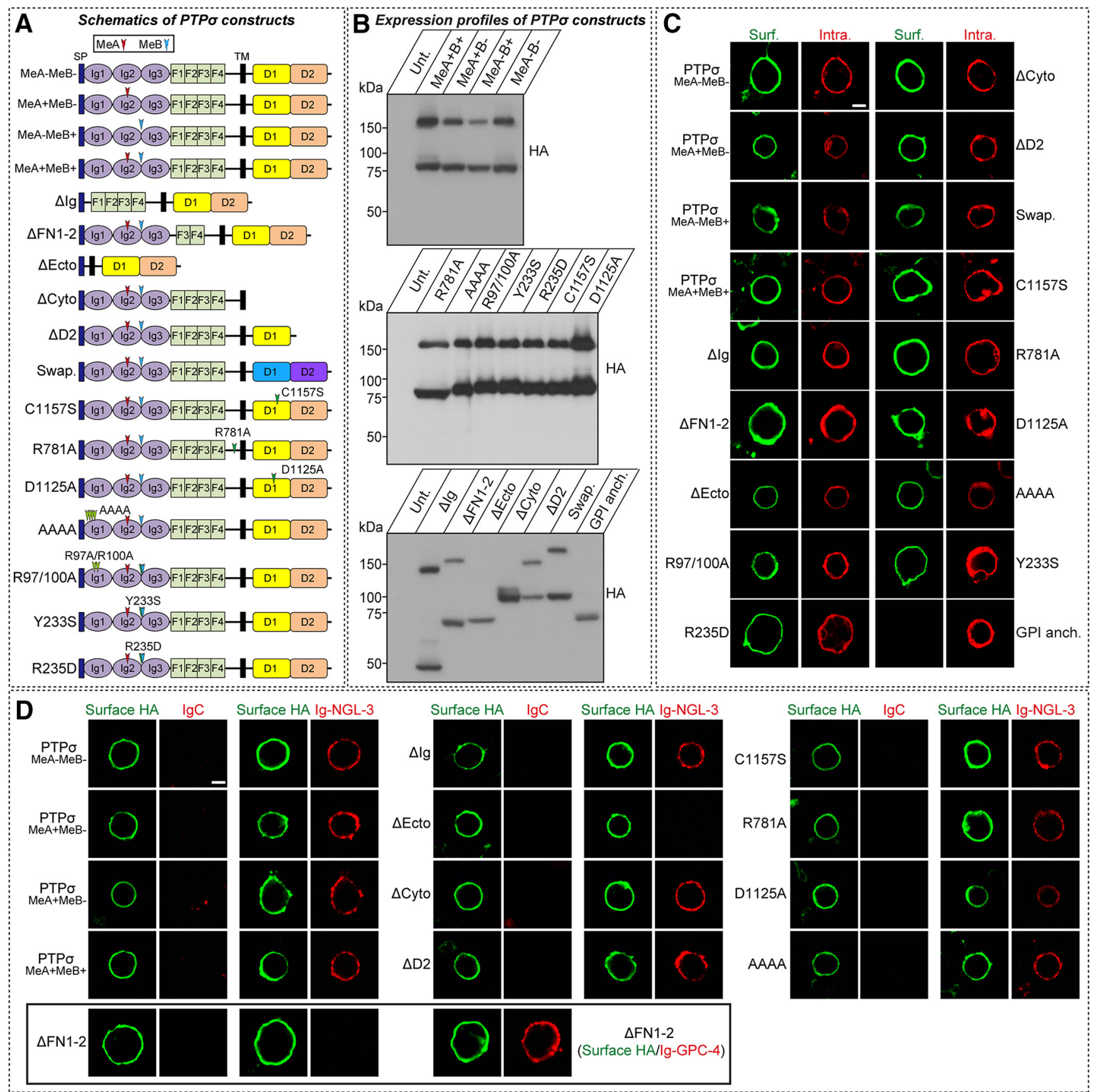

Figure 6. Characterization of total expression, surface trafficking, and ligand-binding properties of various PTP $\sigma$ variants used in the current study. $A, 0$ verview of the PTP $\sigma$ WT and various PTP $\sigma$ variants (alternative splicing variants, deletion mutants, and point mutants). Alternatively spliced variants: PTP $\sigma^{\mathrm{MeA}+\mathrm{MeB}+}, \operatorname{PTP} \sigma$ containing an insert in both MeA and MeB splice sites; PTP $\sigma^{\text {MeA-MeB- }}$, PTP $\sigma$ lacking an insert in both MeA and MeB splice sites; PTP $\sigma^{\text {MeA-MeB+ }}$, PTP $\sigma$ lacking an insert in the MeA splice site and containing an insert in the MeB splice site; and PTP $\sigma^{\text {MeA }+ \text { MeB- }}{ }^{-}$, PTP $\sigma$ containing an insert in the MeA splice site and lacking an insert in the MeB splice site. Deletion variants: PTP $\sigma \Delta \lg$, PTP $\sigma$ with a deletion of the three lg domains; PTP $\sigma$ $\Delta$ FN1-2, PTP $\sigma$ with a deletion of the first two FNIII domains; PTP $\sigma \Delta$ Ecto, PTP $\sigma$ with a deletion of the entire extracellular region; PTP $\sigma \Delta$ Cyto, PTP $\sigma$ with a deletion of the entire intracellular region; and PTP $\sigma \Delta \mathrm{D} 2$, PTP $\sigma$ with a deletion of the intracellular D2 domain. Point mutants: PTP $\sigma$ C1157S or D1125A, tyrosine phosphatase activity-defective mutants of PTP $\sigma$; PTP $\sigma$ R781A, a proteolytic cleavage-defective mutant of PTP $\sigma$; PTP $\sigma$ AAAA, a PTP $\sigma$ mutant in which HS binding was abrogated by replacing four lysines of the first lg domain (K68, K69, K71, and K72) with alanines; PTP $\sigma$ R97/100A, a PTP $\sigma$ mutant in which TrkC binding was abolished by replacing two arginine residues (R97 and R100) with alanines; PTP $\sigma$ Y233S, a PTP $\sigma$ mutant in which binding to both Slitrks and TrkC was eliminated by replacing a tyrosine residue (Y233) with serine; and PTP $\sigma$ R235D, a PTP $\sigma$ mutant in which binding to Slitrks was disrupted by replacing an arginine residue (R235) with aspartic acid. PTP $\sigma$ Swap denotes a hybrid of the PTP $\sigma$ extracellular region with a transmembrane segment and the PTP $\delta$ intracellular region. $\boldsymbol{B}$, Representative immunoblot images from HEK293T cells transfected with HA-tagged PTP $\sigma$ WT or the indicated PTP $\sigma$ variants. Samples containing equal amounts of protein were resolved by SDS-PAGE and immunoblotted using anti-HA antibodies. PTP $\sigma$ GPI denotes a full-length PTP $\sigma$ in which a transmembrane segment was replaced with a GPI anchor sequence. C, Surface expression analysis of HEK293T cells expressing HA-PTP $\sigma$ WT or the indicated HA-tagged PTP $\sigma$ mutant variants. Transfected cells were immunostained with mouse anti-HA antibodies (green) and detected with FITC-conjugated anti-mouse secondary antibodies under nonpermeabilized conditions. (ells were then permeabilized and stained first with rabbit anti-HA antibodies (red) and subsequently with Cy3-conjugated anti-rabbit secondary antibodies. Scale bar (all images), $10 \mu \mathrm{m}$. D, Representative images of cell-surface binding assays. HEK293T cells expressing HA-tagged PTP $\sigma$ WT or the indicated PTP $\sigma$ variants were incubated with $10 \mu \mathrm{g} / \mathrm{ml}$ of control lgC (Fc), Ig-NGL-3 (NGL-3-Fc), or lg-GPC4 (GPC4-Fc) and then analyzed by immunofluorescence imaging of Ig-fusion proteins (red) and HA antibodies (green). Scale bar (all images), $10 \mu \mathrm{m}$. 


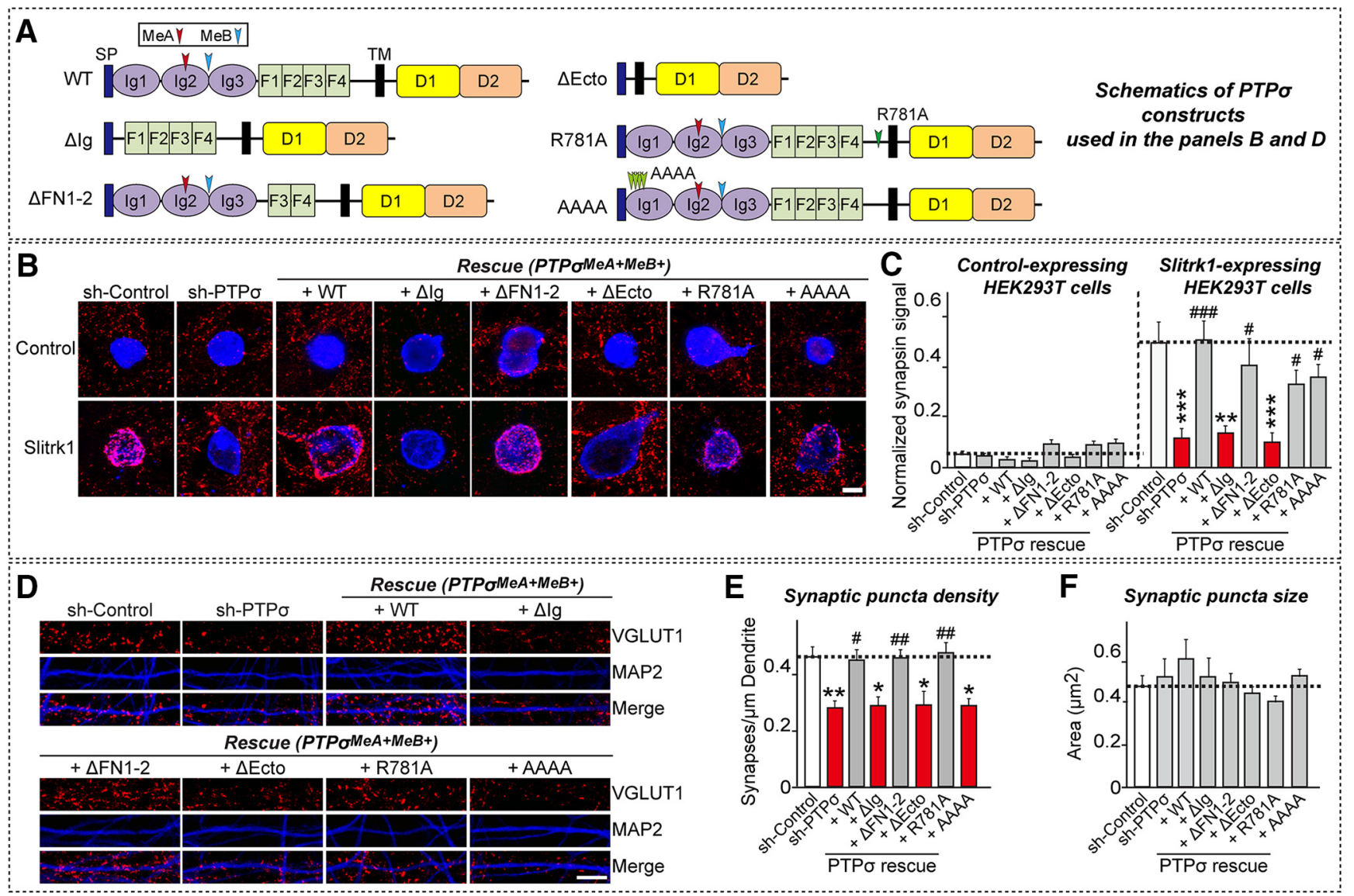

Figure 7. Analysis of PTP $\sigma$ extracellular mechanisms involved in heterologous synapse formation and excitatory synapse development in cultured neurons. $A$, Schematic diagrams of a series of PTP $\sigma$ constructs for deletion variants of extracellular domains or point mutants at extracellular residues. AAAA, Quadruple alanine mutant; D1, first catalytic domain of LAR-RPTPs; D2, second catalytic domain of LAR-RPTPs; F, fibronectin Type III repeat; Ig, Ig domain; MeA, mini-exon A; MeB; mini-exon B; SP, Ig $\kappa$ B signal peptide; TM, transmembrane region. B, Representative images of heterologous synapse-formation activities of PTP $\sigma$ WT and the indicated extracellular domain variants and point mutants. Neurons were infected at DIV4 with lentiviruses expressing sh-Control or sh-PTP $\sigma$, or coexpressing sh-PTP $\sigma$ and the various PTP $\sigma$ mutant constructs (WT, deletion, or point mutants presented in $A$ ), and then cocultured from DIV9 to DIV11 with HEK293T cells expressing EGFP alone (Control) or HA-Slitrk1 (Slitrk1). Neurons were stained with antibodies against EGFP or HA (blue) and synapsin (red). Scale bar (all images), $10 \mu$ m. $C$, Synapse-formation activity in $\boldsymbol{B}$ was quantified by measuring the ratio of synapsin staining intensity (red) to HA/EGFP intensity (blue). Data are mean \pm SEM. ANOVA with a nonparametric Kruskal-Wallis test: ** $p<0.01$; ${ }^{* * *} p<$

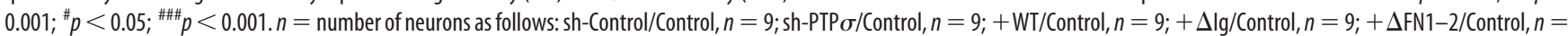
15; $+\Delta$ Ecto/Control, $n=10 ;+$ R781A/Control, $n=11 ;+$ AAAA/Control, $n=10 ;$ sh-Control/Slitrk1, $n=16 ;$ sh-PTP $\sigma / S l i t r k 1, n=18 ;+$ WT/Slitrk1, $n=16 ;+\Delta l g /$ litrk1, $n=12 ;$ $+\Delta \mathrm{FN1} 1-2 /$ Slitrk1, $n=15 ;+\Delta \mathrm{Ecto} /$ Slitrk1, $n=12 ;+$ R781A/Slitrk1, $n=15 ;$ and + AAAA/Slitrk1, $n=14$. $p$ values for Control condition: sh-Control vs sh-PTP $\sigma, p>0.9999 ;$ sh-Control vs + WT, $p>0.9999 ;$ sh-Control vs $+\Delta \mathrm{lg}, p>0.9999 ;$ sh-Control vs $+\Delta \mathrm{FN1}-2, p>0.9999 ;$ sh-Controlvs $+\Delta \mathrm{Ecto,} p>0.9999 ;$ sh-Control vs + R781A, $p=0.3835 ;$ sh-Control vs + AAAA, $p=$ 0.7746 ; sh-PTP $\sigma$ vs + WT, $p>0.9999$; sh-PTP $\sigma$ vs $+\Delta \mathrm{lg}, p>0.9999 ;$ sh-PTP $\sigma$ vs $+\Delta$ FN1-2, $p>0.9999 ;$ sh-PTP $\sigma$ vs $+\Delta$ Ecto, $p>0.9999 ;$ sh-PTP $\sigma$ vs + R781A, $p=0.9262 ;$ and sh-PTP $\sigma$ vs + AAAA, $p>0.9999$. $p$ values for Slitrk1 condition: sh-Control vs sh-PTP $\sigma, p<0.0001$; sh-Control vs + WT, $p>0.9999 ;$ sh-Control vs $+\Delta l g, p=0.008$; sh-Controlvs $+\Delta$ FN1-2, $p>0.9999$; sh-Control vs $+\Delta$ Ecto, $p=0.0002$; sh-Control vs + R781A, $p>0.9999$; sh-Control vs + AAAA, $p>0.9999 ;$ sh-PTP $\sigma$ vs + WT, $p<0.0001$; sh-PTP $\sigma$ vs $+\Delta 19, p>0.9999 ;$ sh-PTP $\sigma$ vs $+\Delta$ FN1-2, $p=0.0348$; sh-PTP $\sigma$ vs $+\Delta$ Ecto, $p>0.9999$; sh-PTP $\sigma$ vs + R781A, $p=0.0341$; and sh-PTP $\sigma$ vs + AAAA, $p=0.0132$. $D$, Representative images from cultured hippocampal neurons infected at DIV4 with lentiviruses expressing sh-Control or sh-PTP $\sigma$, or coinfected with lentiviruses expressing sh-PTP $\sigma$ and the indicated rescue viruses for PTP $\sigma$ WT and extracellular domain mutants and analyzed at DIV14 by double-immunofluorescence detection of MAP2 (blue) and the excitatory synaptic marker VGLUT1 (red). Scale bar (all images), $10 \mu \mathrm{m} . \boldsymbol{E}, \boldsymbol{F}$, Summary graphs of the effects of PTP $\sigma$ molecular replacement in neurons on puncta density $(\boldsymbol{E})$ and puncta size $(\boldsymbol{F})$, measured using VGLUT1 as an excitatory synaptic marker. Two or three dendrites per transfected neuron were analyzed and group-averaged. Data are mean \pm SEM. ANOVA with a nonparametric Kruskal-Wallis test: ${ }^{*} p<0.05 ;{ }^{* *} p<$ 0.01; ${ }^{p} p<0.05 ; \#$; $p<0.01 . n=$ number of neurons as follows: sh-Control, $n=28 ;$ sh-PTP $\sigma, n=20 ;+$ WT, $n=20 ;+\Delta \mathrm{lg}, n=20 ;+\Delta$ FN1-2, $n=21 ;+\Delta$ Ecto, $n=20 ;+$ R781A, $n=14$; and + AAAA, $n=20$. $p$ values for puncta density: sh-Control vs sh-PTP $\sigma, p=0.0063$; sh-Control vs + WT, $p>0.9999$; sh-Control vs $+\Delta \mathrm{lg}, p=0.0178$; sh-Control vs $+\Delta$ FN1-2, $p>0.9999 ;$ sh-Control vs $+\Delta \mathrm{Ect0}, p=0.0493$; sh-Control vs + R781A, $p>0.9999 ;$ sh-Control vs + AAAA, $p=0.0132 ;$ sh-PTP $\sigma \mathrm{vs}+$ WT, $p=0.0281 ;$ sh-PTP $\sigma \mathrm{vs}+\Delta \mathrm{lg}$, $p>0.9999$; sh-PTP $\sigma$ vs $+\Delta$ FN1-2, $p=0.0073$; sh-PTP $\sigma$ vs $+\Delta$ Ecto, $p>0.9999$; sh-PTP $\sigma$ vs + R781A, $p=0.0086$; and sh-PTP $\sigma$ vs + AAAA, $p>0.9999 . p$ values for puncta sizes: sh-Control vs sh-PTP $\sigma, p>0.9999 ;$ sh-Control vs + WT, $p>0.9999 ;$ sh-Control vs $+\Delta l g, p>0.9999 ;$ sh-Control vs $+\Delta \mathrm{FN} 1-2, p>0.9999 ;$ sh-Control vs $+\Delta \mathrm{Ecto}, p>0.9999 ;$ sh-Control vs + R781A, $p>0.9999 ;$ sh-Control vs + AAAA, $p>0.9999$; sh-PTP $\sigma$ vs + WT, $p>0.9999 ;$ sh-PTP $\sigma$ vs $+\Delta l g, p>0.9999 ;$ sh-PTP $\sigma$ vs $+\Delta$ FN1-2, $p>0.9999 ;$ sh-PTP $\sigma$ vs $+\Delta$ Ecto, $p>0.9999 ;$ sh-PTP $\sigma$ vs + R781A, $p>0.9999 ;$ and sh-PTP $\sigma$ vs + AAAA, $p>0.9999$.

synaptic assembly, how PTP $\sigma$ specifically exerts its action toward the excitatory synapse type remains to be determined. LAR-RPTP family members are structurally similar, sharing $64 \%$ overall amino acid sequence identity (Pulido et al., 1995; Um and Ko, 2013). In particular, the D2 domain of human PTP $\sigma$ and PTP $\delta$ exhibits 95\%-99\% similarity/identity (data not shown). Therefore, we were initially skeptical about the contribution of intra- cellular mechanisms to determining the specific actions of PTP $\sigma$ and PTP $\delta$ in driving specific types of synapse assembly, especially given that the suggested role of PTP $\sigma$ and PTP $\delta$ in driving excitatory and inhibitory synapse assembly, respectively, was still hypothetical. We thus expected that replacing the intracellular region of PTP $\sigma$ with its PTP $\delta$ counterpart would not compromise the ability of PTP $\sigma$ to mediate heterologous synapse 


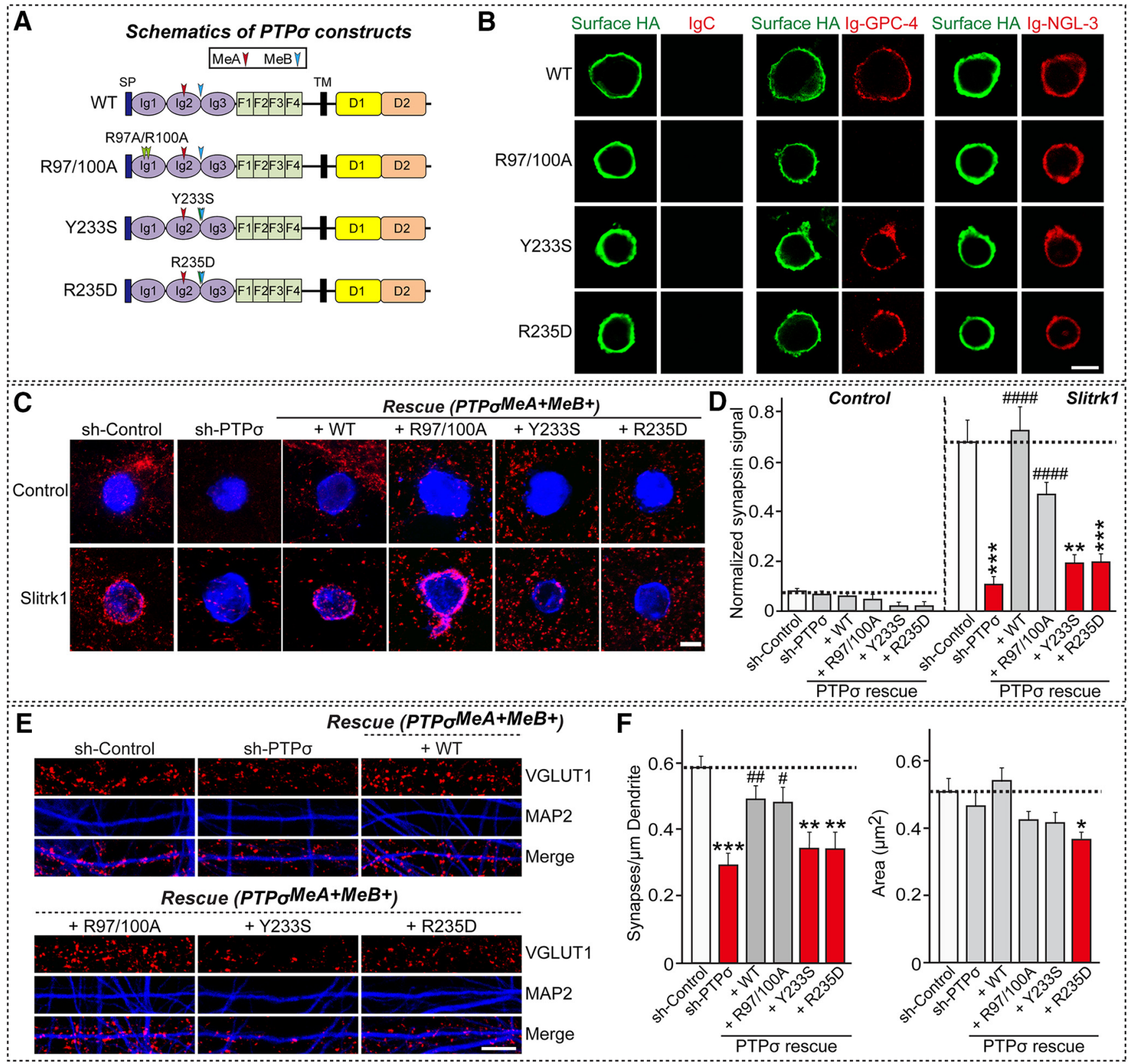

Figure 8. Analysis of the effects of PTP $\sigma$ extracellular point mutants that selectively abolish interactions with TrkC or Slitrks on heterologous synapse formation. $A$, Schematic illustration of PTP $\sigma$ WT and point mutants that exhibit defective binding to the specific postsynaptic ligands, TrkC or Slitrks. D1, First catalytic domain of LAR-RPTPs; D2, second catalytic domain of LAR-RPTPs; F, fibronectin Type III repeat; Ig, Ig domain; MeA, mini-exon A; MeB; mini-exon B; SP, Ig $\kappa$ B signal peptide; TM, transmembrane region. B, Representative images of cell surface-binding assays. HEK293T cells expressing HA-tagged PTP $\sigma$ WT or its various point mutants were incubated with $10 \mu \mathrm{g} / \mathrm{ml}$ control lgC (Control), lg-GPC-4, or lg-NGL-3, and then analyzed by immunofluorescence imaging of Ig-fusion proteins (red) and HA antibodies (green). Scale bar (all images), $10 \mu \mathrm{m}$. C, Representative images of the heterologous synapse-formation activities of PTP $\sigma$ WT and specific ligand-binding-defective mutants. Neurons were infected at DIV4 with lentiviruses expressing sh-Control or sh-PTP $\sigma$, or coexpressing various PTP $\sigma$ mutant constructs (WT and ligand-bindingdefective mutants presented in $\boldsymbol{A}$ ), and then cocultured from DIV9 to DIV11 with HEK293T cells expressing EGFP alone (Control) or HA-Slitrk1 (Slitrk1). Neurons were stained with antibodies against EGFP or HA (blue) and synapsin (red). Scale bar (all images), $10 \mu \mathrm{m}$. D. The synapse-formation activity in ( was quantified by measuring the ratio of synapsin staining intensity (red) to HA/EGFP intensity (blue). Data are mean \pm SEM. ANOVA with a nonparametric Kruskal-Wallis test: ${ }^{* *} p<0.01 ;{ }^{* * *} p<0.001 ;{ }^{\# \# \# p}<0.0001 . n=$ number of neurons as follows: sh-Control/Slitrk1, $n=$ 19; sh-PTP $\sigma /$ Control, $n=15 ;+$ WT/Control, $n=16 ;+$ R97/100A/Control, $n=10 ;+$ Y233S/Control, $n=9$; and +R235D/Control, $n=10 ;$ sh-Control/Slitrk1, $n=20 ;$ sh-PTP $\sigma /$ Slitrk1, $n=25 ;$ + WT/Slitrk1, $n=25 ;+$ R97/100A/Slitrk1, $n=22 ;+$ Y233S/Slitrk1, $n=16$; and +R235D/Slitrk1, $n=24$. $p$ values for Control condition: sh-Control vs sh-PTP $\sigma, p>0.9999 ;$ sh-Control vs + WT,

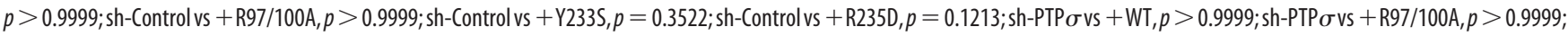
sh-PTP $\sigma$ vs + Y233S, $p=0.1596$; and sh-PTP $\sigma$ vs + R235D, $p=0.0504$. $p$ values for Slitrk1 condition: sh-Control vs sh-PTP $\sigma, p<0.0001$; sh-Control vs + WT, $p>0.9999$; sh-Control vs + R97/100A, $p>0.9999$; sh-Control vs + Y233S, $p=0.0015$; sh-Control vs + R235D, $p=0.0004$; sh-PTP $\sigma$ vs + WT, $p<0.0001$; sh-PTP $\sigma$ vs + R97/100A, $p<0.0001 ;$ sh-PTP $\sigma$ vs + Y233S, $p>0.9999$; and sh-PTP $\sigma$ vs + R235D, $p>0.9999$. E, Representative images of cultured hippocampal neurons infected at DIV4 with lentiviruses expressing sh-Control or sh-PTP $\sigma$, or coinfected with lentiviruses expressing sh-PTP $\sigma$ and the indicated rescue viruses for human PTP $\sigma$ alternative splicing variants. These images were taken on DIV14 following double-immunofluorescence labeling of MAP2 (blue) and the excitatory synaptic marker VGLUT1 (red). Scale bar (all images), $10 \mu \mathrm{m}$. $F$, Summary graphs of the effects of PTP $\sigma$ molecular replacement in neurons on puncta density (left) and puncta size (right), measured using VGLUT1 as an excitatory synaptic marker. Two or three dendrites per transfected neuron were analyzed and group-averaged. Data are mean \pm SEM. ANOVA with a nonparametric Kruskal-Wallis test: ${ }^{* *} p<0.01 ;{ }^{* * *} p<0.001 ;{ }^{\#} p<0.05 ;{ }^{\# \#} p<0.01 . n=$ number of neurons as follows: sh-Control, $n=21 ;$ sh-PTP $\sigma, n=20 ;+$ WT, $n=$ $16 ;+\mathrm{R} 97 / 100 \mathrm{~A}, n=21 ;+\mathrm{Y} 233 \mathrm{~S}, n=15 ;$ and $+\mathrm{R} 235 \mathrm{D}, n=15 . p$ values for puncta density: sh-Control vs sh-PTP $\sigma, p<0.0001$; sh-Control vs +WT, $p>0.9999 ;$ sh-control vs + R97/100A, $p>0.9999$; sh-Control vs + Y233S, $p=0.0036$; sh-Control vs + R235D, $p=0.0059$; sh-PTP $\sigma$ vs + WT, $p=0.0074$; sh-PTP $\sigma$ vs + R97/100A, $p=0.0179$; (Figure legend continues.) 


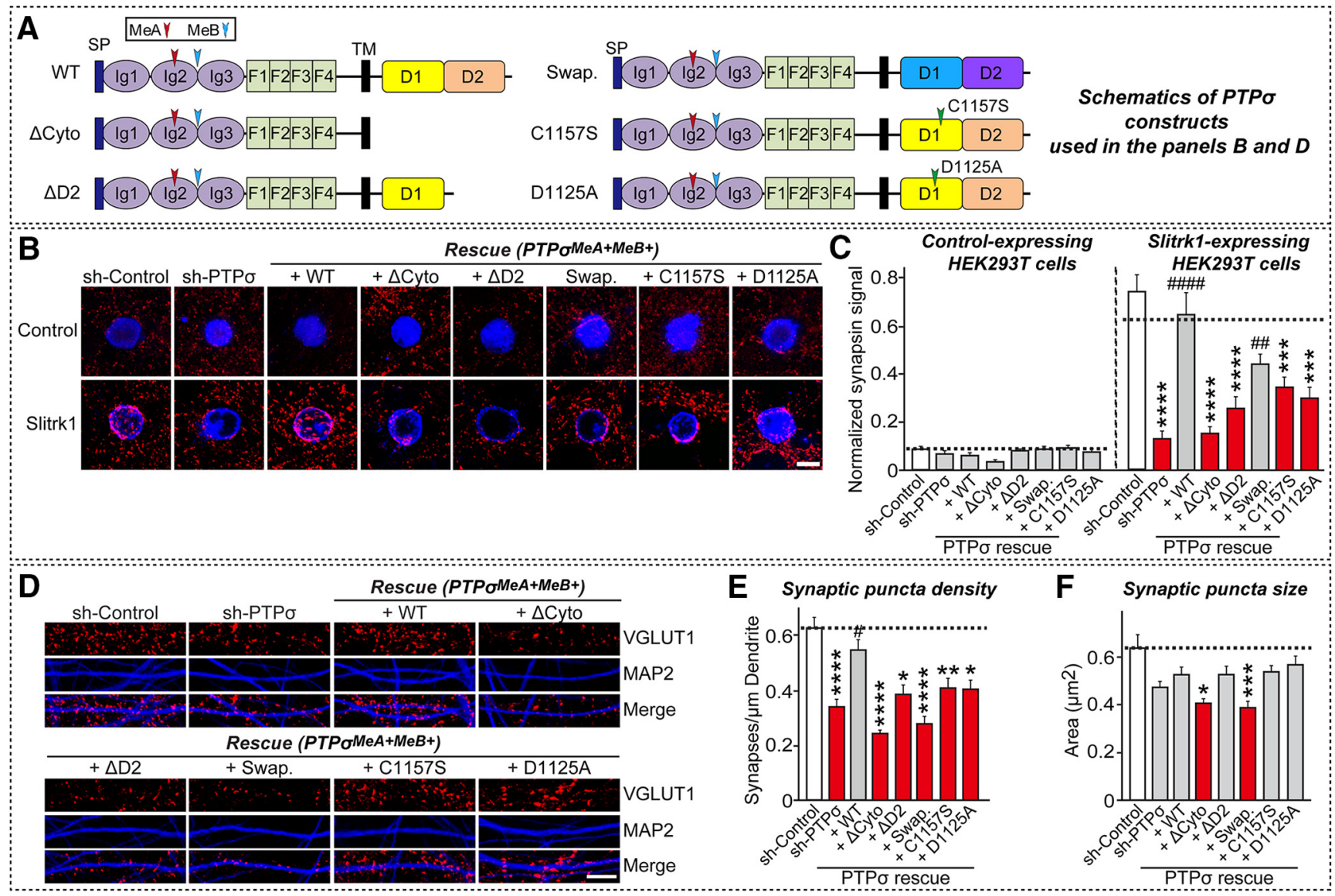

Figure 9. Analysis of PTP $\sigma$ intracellular mechanisms involved in heterologous synapse formation and excitatory synapse development in cultured neurons. $A$, Schematic diagrams of a series of PTP $\sigma$ constructs for deletion variants of intracellular domains or point mutants at intracellular residues. Cyto, Cytoplasmic; D1, first catalytic domain of LAR-RPTPs; D2, second catalytic domain of LAR-RPTPS; F, fibronectin Type III repeat; Ig, Ig domain; MeA, mini-exon A; MeB; mini-exon B; SP, Ig $\kappa$ B signal peptide; TM, transmembrane region. $B$, Representative images of the heterologous synapse-formation activities of PTP $\sigma$ WT and intracellular domain mutants. Neurons were infected at DIV4 with lentiviruses expressing sh-Control or sh-PTP $\sigma$, or coexpressing sh-PTP $\sigma$ and the various PTP $\sigma$ variants (WT, deletion variants, and point mutants presented in $A$ ), and then cocultured from DIV9 to DIV11 with HEK293T cells expressing EGFP alone (Control) or HA-Slitrk1 (Slitrk1). Neurons were stained with antibodies against EGFP or HA (blue) and synapsin (red). Scale bar (all images), $10 \mu \mathrm{m}$. $\boldsymbol{C}$, The synapse-formation activity in $\boldsymbol{B}$ was quantified by measuring the ratio of synapsin staining intensity (red) to HA/EGFP intensity (blue). Data are mean \pm SEM. ANOVA with a nonparametric Kruskal-Wallis test: ${ }^{* * *} p<0.001 ;{ }^{* * * *} p<0.0001$; ${ }^{\# \#} p<0.01$; ${ }^{\# \# \# \#} p<$ 0.0001. $n=$ number of neurons as follows: sh-Control/Control, $n=19 ;$ sh-PTP $\sigma /$ Control, $n=15 ;+$ WT/Control, $n=16 ;+\Delta$ Cyto/Control, $n=9 ;+\Delta D 2 /$ Control, $n=10 ;+$ Swap/Control, $n=$ 11; +C1157S/Control, $n=10 ;$ and +D1125A/Control, $n=10 ;$ sh-Control/Slitrk1, $n=15 ;$ sh-PTP $\sigma / S$ litrk1, $n=15 ;+$ WT/Slitrk1, $n=15 ;+\Delta$ Cyto/Slitrk1, $n=16 ;+\Delta \mathrm{D} 2 /$ Slitrk1, $n=14 ;$ + Swap/Slitrk1, $n=21 ;+C 1157$ S/Slitrk1, $n=21$; and +D1125A/Slitrk1, $n=22$. $p$ values for Control condition: sh-Control vs sh-PTP $\sigma, p>0.9999 ;$ sh-Control vs +WT, $p>0.9999 ;$ sh-Control vs $+\Delta$ Cyto,$p=0.2621$; sh-Controlvs $+\Delta \mathrm{D} 2, p=0.0915$; sh-Controlvs + Swap, $p>0.9999$; sh-Control vs + C1157S, $p>0.9999$; sh-Controlvs + D1125A, $p>0.9999 ;$ sh-PTP $\sigma$ vs + WT, $p>$ 0.9999; sh-PTP $\sigma$ vs $+\Delta$ Cyto, $p>0.9999 ;$ sh-PTP $\sigma v s+\Delta D 2, p>0.9999 ;$ sh-PTP $\sigma v s+$ Swap, $p>0.9999 ;$ sh-PTP $\sigma v s+C 11575, p>0.9999 ;$ and sh-PTP $\sigma v s+D 1125 A, p>0.9999 . p$ values for Slitrk1 condition: sh-Control vs sh-PTP $\sigma, p<0.0001$; sh-Control vs + WT, $p>0.9999$; sh-Control vs $+\Delta$ Cyto, $p<0.0001$; sh-Controlvs $+\Delta D 2$, $p=0.002$; sh-Control vs + Swap, $p>0.9999$; sh-Control vs + C11575, $p=0.0445$; sh-Control vs + D1125A, $p=0.0045$; sh-PTP $\sigma$ vs + WT, $p<0.0001$; sh-PTP $\sigma$ vs $+\Delta$ Cyto, $p>0.9999 ;$ sh-PTP $\sigma$ vs $+\Delta$ D2, $p>0.9999 ;$ sh-PTP $\sigma$ vs + Swap, $p=0.0023$; sh-PTP $\sigma$ vs + C1157S, $p=0.2585$; and sh-PTP $\sigma$ vs + D1125A, $p=0.6586$. D. Representative images of cultured hippocampal neurons infected at DIV4 with lentiviruses expressing sh-Control or sh-PTP $\sigma$, or coinfected with lentiviruses expressing sh-PTP $\sigma$ and the indicated rescue viruses for PTP $\sigma$ WT and intracellular domain mutants. Images were obtained on DIV14 following double-immunofluorescence labeling of MAP2 (blue) and the excitatory synaptic marker VGLUT1 (red). Scale bar (all images), $10 \mu \mathrm{m} . \boldsymbol{E}, \boldsymbol{F}$, Summary graphs of the effects of PTP $\sigma$ molecular replacement in neurons on puncta density $(\boldsymbol{E})$ and puncta size $(\boldsymbol{F})$, measured using VGLUT1 as an excitatory synaptic marker. Two or three dendrites per transfected neuron were analyzed and group-averaged. Data are mean \pm SEM. ANOVA with a nonparametric Kruskal-Wallis test: ${ }^{*} p<0.05 ;{ }^{* *} p<0.01 ;{ }^{* * *} p<$ $0.001 ;{ }^{* * * *} p<0.0001 ;{ }^{\#} p<0.05 . n=$ number of neurons as follows: sh-Control, $n=17 ;$ sh-PTP $\sigma, n=21 ;+$ WT, $n=15 ;+\Delta$ Cyto, $n=15 ;+\Delta \mathrm{D} 2, n=14 ;+$ SWAP, $n=20$; + C1157S, $n=25$; and +D1125A, $n=26$. $p$ values for puncta density: sh-Control vs sh-PTP $\sigma, p<0.0001$; sh-Control vs + WT, $p>0.9999$; sh-Control vs $+\Delta$ Cyto, $p<0.0001$; sh-Control vs $+\Delta \mathrm{D} 2, p=0.0184$; sh-Control vs + Swap, $p<0.0001$; sh-Control vs + C1157S, $p=0.0083$; sh-Control vs + D1125A, $p=0.0147$; sh-PTP $\sigma$ vs + WT, $p=0.0105$; sh-PTP $\sigma$ vs $+\Delta$ Cyto, $p>0.9999 ;$ sh-PTP $\sigma$ vs $+\Delta$ D2, $p>0.9999$; sh-PTP $\sigma$ vs + Swap, $p>0.9999 ;$ sh-PTP $\sigma$ vs + C1157s, $p>0.9999 ;$ and sh-PTP $\sigma$ vs + D1125A, $p>0.9999$. $p$ values for puncta size: sh-Control vs sh-PTP $\sigma, p>0.9999$; sh-Control vs + WT, $p>0.9999$; sh-Control vs $+\Delta$ Cyto, $p<0.0161$; sh-Control vs $+\Delta D 2, p>0.9999$; sh-Control vs + Swap, $p=0.0003$; sh-Control vs + C1157S, $p>0.9999 ;$ sh-Control vs + D1125A, $p>0.9999 ;$ sh-PTP $\sigma$ vs + WT, $p>0.9999 ;$ sh-PTP $\sigma$ vs $+\Delta$ Cyto, $p>0.9999 ;$ sh-PTP $\sigma$ vs $+\Delta$ D2, $p>0.9999 ;$ sh-PTP $\sigma$ vs + Swap, $p=0.266$; sh-PTP $\sigma$ vs + C1157S, $p>0.9999 ;$ and sh-PTP $\sigma$ vs + D1125A, $p>0.9999$.

$\leftarrow$

(Figurelegend continued.) sh-PTP $\sigma \mathrm{vs}+\mathrm{Y} 233 \mathrm{~S}, p>0.9999$; and sh-PTP $\sigma \mathrm{vs}+\mathrm{R} 235 \mathrm{D}, p>$ 0.9999. $p$ values for puncta size: sh-Control vs sh-PTP $\sigma, p>0.9999 ;$ sh-Control vs + WT, $p>$ 0.9999; sh-Controlvs + R97/100A, $p>0.9999$; sh-Control vs + Y2335, $p>0.9999$; sh-Control vs + R235D, $p=0.0177$; sh-PTP $\sigma$ vs + WT, $p>0.9999$; sh-PTP $\sigma$ vs + R97/100A, $p>$ 0.9999; sh-PTP $\sigma$ vs + Y233S, $p>0.9999 ;$ and sh-PTP $\sigma$ vs + R235D, $p=0.2712$. formation. Using Slitrk6, a Slitrk isoform that induces inhibitory heterologous synapse formation more potently than Slitrk1, we performed heterologous synapse-formation assays in PTP $\sigma$ - or PTP $\delta$-deficient neurons. We previously reported that PTP $\delta$ KD specifically impaired the inhibitory, but not excitatory, heterologous synapse formation activity induced by Slitrk6, as we previously reported (Yim et al., 2013). Strikingly, reexpression of 


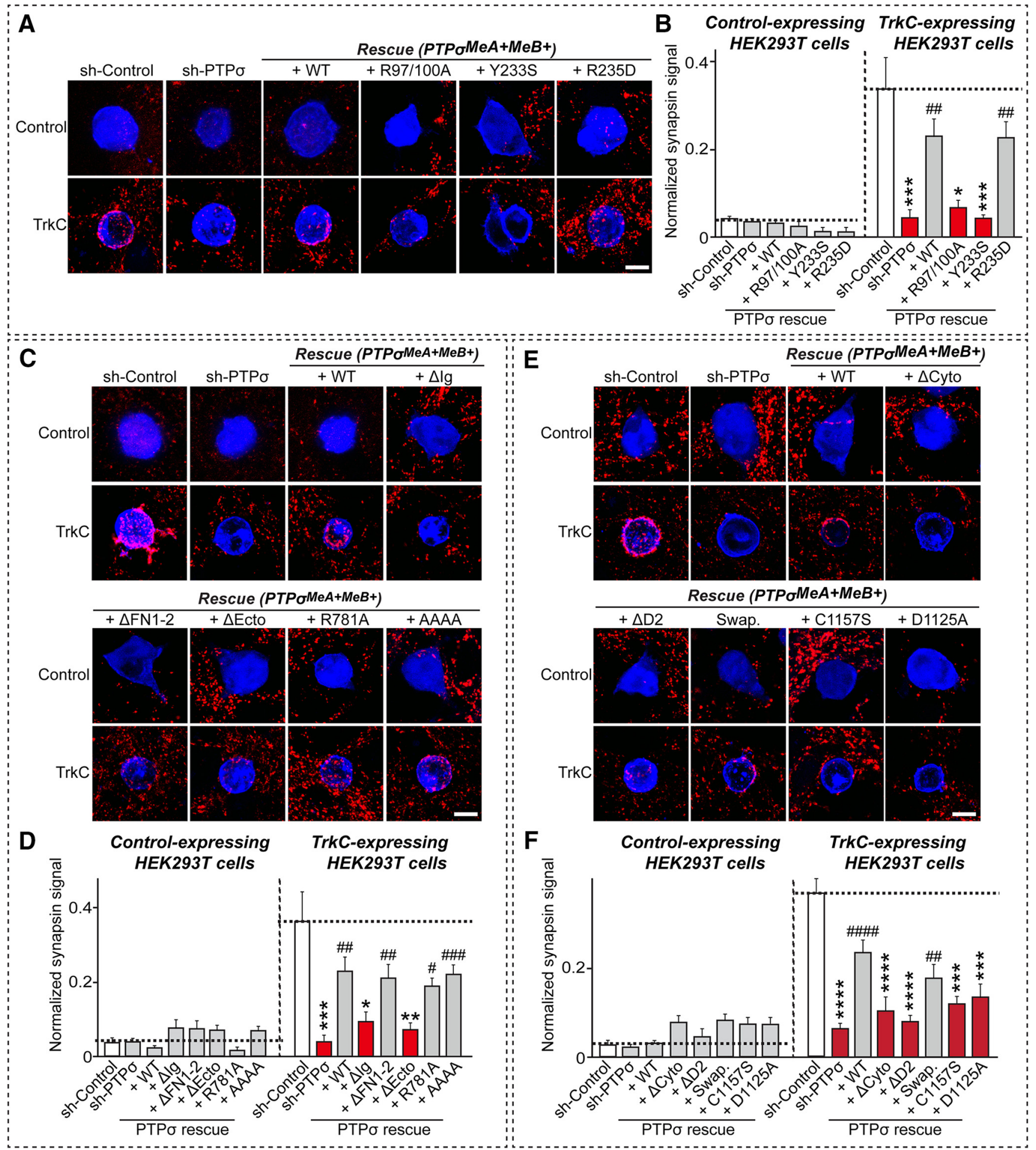

Figure 10. Analysis of PTP $\sigma$ extracellular and intracellular mechanisms involved in heterologous synapse formation induced by TrkC. A, Representative images of the heterologous synapseformation activities of PTP $\sigma$ WT and the indicated specific ligand binding-defective point mutants. Neurons were infected at DIV4 with lentiviruses expressing sh-Control or sh-PTP $\sigma$, or coexpressing sh-PTP $\sigma$ and various PTP $\sigma$ mutant constructs presented in Figure 6, and then cocultured from DIV9 to DIV11 with HEK293T cells expressing EGFP alone (Control) or HA-TrkC (TrkC). Neurons were stained with antibodies against EGFP or HA (blue) and synapsin (red). Scale bar (all images), $10 \mu \mathrm{m}$. B, The synapse-formation activity in $\boldsymbol{A}$ was quantified by measuring the ratio of synapsin staining intensity (red) to HA/EGFP intensity (blue). Data are mean \pm SEM. ANOVA with a nonparametric Kruskal-Wallis test: ${ }^{*} p<0.05 ;{ }^{* * *} p<0.001 ;{ }^{* \#} p<0.01$. $n=$ number of neurons as follows: sh-Control/Control, $n=8 ;$ sh-PTP $\sigma /$ Control, $n=11 ;+$ WT/Control, $n=11 ;+$ R97/100A/Control, $n=9 ;+\mathrm{Y} 233 \mathrm{~S} /$ Control, $n=9$; and + R235D/Control, $n=12 ;$ sh-Control/TrkC, $n=$ 13; sh-PTP $\sigma /$ TrkC, $n=11 ;+$ WT/TrkC, $n=13 ;+$ R97/100A/TrkC, $n=11 ;+\mathrm{Y} 233 \mathrm{~S} / \mathrm{TrkC}, n=16 ;$ and + R235D/TrkC, $n=13 . p$ values for Control condition: sh-Control vs sh-PTP $\sigma, p>0.9999 ;$ sh-Control vs + WT, $p>0.9999$; sh-Control vs + R97/100A, $p=0.2309$; sh-Control vs + Y233S, $p=0.3307$; sh-Control vs + R235D, $p>0.9999 ;$ sh-PTP $\sigma$ vs + WT, $p>0.9999 ;$ sh-PTP $\sigma$ vs $+\mathrm{R} 97 / 100 \mathrm{~A}, p=0.0577$; sh-PTP $\sigma$ vs + Y233S, $p=0.0814$; and sh-PTP $\sigma$ vs + R235D, $p=0.0504$. $p$ values for TrkC condition: sh-Control vs sh-PTP $\sigma, p=0.0004 ;$ sh-Control vs + WT, $p>$ 0.9999; sh-Control vs + R97/100A, $p=0.0153$; sh-Control vs + Y233S, $p=0.0003$; sh-Control vs + R235D, $p>0.9999 ;$ sh-PTP $\sigma$ vs + WT, $p=0.002 ;$ sh-PTP $\sigma$ vs + R97/100A, $p>0.9999$; sh-PTP $\sigma$ vs + Y 233S, $p>0.9999$; and sh-PTP $\sigma$ vs + R235D, $p=0.001$. C, Representative images of the heterologous synapse-formation activities of PTP $\sigma$ WT and the indicated extracellular domain variants and point mutants. Neurons were infected at DIV4 with lentiviruses expressing sh-Control or sh-PTP $\sigma$, or coexpressing sh-PTP $\sigma$ and various PTP $\sigma$ mutant constructs (WT, deletion, or point mutants presented in Fig. 6), and then cocultured from DIV9 to DIV11 with HEK293T cells expressing EGFP alone (Control) or HA-TrkC (TrkC). Neurons were (Figure legend continues.) 
PTP $\sigma$ WT or a PTP $\sigma$ intracellularly swapped variant composed of the PTP $\sigma$ extracellular region and PTP $\delta$ intracellular region $(\mathrm{PTP} \sigma$ Swap) rescued the defective synaptogenic activity of PTP $\sigma$-deficient neurons (Fig. 12 B,D). Moreover, reintroduction of the PTP $\sigma$ Swap mutant into PTP $\delta$-deficient neurons also restored the impaired heterologous inhibitory synapse formation observed in $\mathrm{PTP} \delta$-deficient neurons (Fig. $12 B, C$ ). These data suggest that Slitrk6 induces presynaptic assembly of both excitatory and inhibitory synapses, provided that interactions with extracellular ligands (i.e., PTP $\sigma$ or PTP $\delta$ ) are permissive, regardless of the identity of intracellular signaling pathways. Notably, lentiviral expression of the PTP $\sigma$ Swap mutant failed to maintain the density of excitatory synapse puncta at a level comparable with that supported by PTP $\sigma$ WT (Fig. 9). In control experiments, neither PTP $\sigma$ KD nor PTP $\delta$ KD affected the heterologous synapse-formation activity induced by NL-2 (Fig. 12B-D). Collectively, these data suggest that the PTP $\sigma$ D2 domain per se does not dictate the identity of specific synapse types during heterologous synapse formation mediated by LAR-RPTPs, but that a

\section{$\longleftarrow$}

(Figure legend continued.) stained with antibodies against EGFP or HA (blue) and synapsin (red). Scale bar (all images), $10 \mu \mathrm{m}$. D, The synapse-formation activity in $\mathbf{C}$ was quantified by measuring the ratio of synapsin staining intensity (red) to HA/EGFP intensity (blue). Data are mean \pm SEM. ANOVA with a nonparametric Kruskal-Wallis test: ${ }^{*} p<0.05$; ${ }^{* *} p<0.01$; ${ }^{* * *} p<0.001 ;{ }^{\#} p<0.05 ;{ }^{\# \#} p<0.01 ;{ }^{\# \# \#} p<0.001 . n=$ number of neurons as follows: sh-Control/Control, $n=8 ;$ sh-PTP $\sigma /$ Control, $n=11 ;+$ WT/Control, $n=11 ;+\Delta \mathrm{lg} /$ Control, $n=11 ;+\Delta$ FN1-2/Control, $n=10 ;+\Delta$ Ecto/Control, $n=10 ;+$ R781A/Control, $n=10$; and + AAAA/Control, $n=12$; sh-Control/TrkC, $n=12$; sh-PTP $\sigma /$ TrkC, $n=11 ;+$ WT/TrkC, $n=13 ;+\Delta \mathrm{lg} / \mathrm{TrkC}, n=13 ;+\Delta \mathrm{FN} 1-2 / \mathrm{TrkC}, n=13 ;+\Delta \mathrm{Ecto} / \mathrm{TrkC}, n=11 ;+\mathrm{R} 781 \mathrm{~A} /$ TrkC, $n=14$; and + AAAA/TrkC, $n=14$. $p$ values for Control condition: sh-Control vs sh-PTP $\sigma$, $p>0.9999$; sh-Control vs + WT, $p>0.9999$; sh-control vs $+\Delta l g, p>0.9999$; sh-Control vs $+\Delta \mathrm{FN1}-2, p>0.9999 ;$ sh-Control vs $+\Delta \mathrm{Ect0}, p>0.9999$; sh-Control vs + R781A, $p>$ 0.9999 ; sh-Control vs + AAAA, $p>0.9999$; sh-PTP $\sigma \mathrm{vs}+$ WT, $p>0.9999 ;$ sh-PTP $\sigma \mathrm{vs}+\Delta \mathrm{lg}$, $p>0.9999 ;$ sh-PTP $\sigma$ vs $+\Delta$ FN1-2, $p>0.9999 ;$ sh-PTP $\sigma$ vs $+\Delta$ Ecto, $p>0.9999 ;$ sh-PTP $\sigma$ vs + R781A, $p>0.9999$; and sh-PTP $\sigma$ vs + AAAA, $p>0.9999$. $p$ values for TrkC condition: sh-Control vs sh-PTP $\sigma, p=0.0003$; sh-Control vs + WT, $p>0.9999$; sh-Control vs $+\Delta \mathrm{lg}$, $p=0.0374$; sh-Control vs $+\Delta \mathrm{FN1}-2, p>0.9999$; sh-Control vs $+\Delta \mathrm{Ecto}, p=0.0085$; sh-Control vs + R781A, $p>0.9999$; sh-Control vs + AAAA, $p>0.9999$; sh-PTP $\sigma$ vs +WT, $p=0.0027$; sh-PTP $\sigma$ vs $+\Delta \mathrm{lg}, p>0.9999 ;$ sh-PTP $\sigma \mathrm{vs}+\Delta$ FN1 $-2, p=0.0082 ;$ sh-PTP $\sigma \mathrm{vs}$ $+\Delta$ Ecto, $p>0.9999$; sh-PTP $\sigma$ vs + R781A, $p=0.0112$; and sh-PTP $\sigma$ vs + AAAA, $p=$ 0.0006 . E, Representative images of the heterologous synapse-formation activities of PTP $\sigma$ WT and the indicated intracellular domain variants and point mutants. Neurons were infected at DIV4 with lentiviruses expressing sh-Control or sh-PTP $\sigma$, or coexpressing sh-PTP $\sigma$ and the indicated PTP $\sigma$ mutant constructs presented in Figures 9 and 11 , and then cocultured from DIV 9 to DIV11 with HEK293T cells expressing EGFP alone (Control) or HA-TrkC (TrkC). Neurons were stained with antibodies against EGFP or HA (blue) and synapsin (red). Scale bar (all images), 10 $\mu \mathrm{m} . \boldsymbol{F}$, The synapse-formation activity in $\boldsymbol{E}$ was quantified by measuring the ratio of synapsin staining intensity (red) to HA/EGFP intensity (blue). Data are mean \pm SEM. ANOVA with a nonparametric Kruskal-Wallis test: ${ }^{* * *} p<0.001$; ${ }^{* * *} p<0.0001$; ${ }^{\# \#} p<0.01$; ${ }^{\# \# \# \# p<}$ 0.0001. $n=$ number of neurons as follows: sh-Control/Control, $n=8 ;$ sh-PTP $\sigma /$ Control, $n=$ $11 ;+$ WT/Control, $n=11 ;+\Delta$ Cyto/Control, $n=11 ;+\Delta \mathrm{D} 2 /$ Control, $n=9 ;+$ Swap/ Control, $n=8 ;+C 1157 \mathrm{~S} /$ Control, $n=9$; and + D1125A/Control, $n=12 ;$ sh-Control/TrkC, $n=21 ;$ sh-PTP $\sigma /$ TrkC, $n=28 ;+$ WT/TrkC, $n=21 ;+\Delta$ Cyto/TrkC, $n=12 ;+\Delta \mathrm{D} 2 /$ TrkC, $n=13 ;+$ Swap/TrkC, $n=17 ;+C 1157 S / T r k C, n=20 ;$ and + D1125A, $n=15 . p$ values for Control condition: sh-Control vs sh-PTP $\sigma, p>0.9999$; sh-Control vs + WT, $p>0.9999$; shControl vs $+\Delta$ Cyto, $p>0.9999$; sh-Control vs $+\Delta \mathrm{D} 2, p>0.9999$; sh-Control vs + Swap, $p=0.4602$; sh-Control vs + C1157S, $p=0.6221$; sh-Control vs + D1125A, $p>0.9999$; sh-PTP $\sigma$ vs + WT, $p>0.9999 ;$ sh-PTP $\sigma$ vs $+\Delta$ Cyto, $p=0.4431$; sh-PTP $\sigma$ vs $+\Delta \mathrm{D} 2, p=$ 0.6263; sh-PTP $\sigma$ vs + Swap, $p=0.1718$; sh-PTP $\sigma$ vs $+C 11575, p=0.2347$; and sh-PTP $\sigma$ vs + D1125A, $p>0.9999$. $p$ values for TrkC condition: sh-Control vs sh-PTP $\sigma, p<0.0001$; sh-Control vs + WT, $p>0.9999$; sh-Control vs $+\Delta$ Cyto, $p<0.0001$; sh-Control vs $+\Delta \mathrm{D} 2$, $p<0.0001$; sh-Control vs + Swap, $p=0.0539$; sh-Control vs + C1157S, $p=0.0002$; shControl vs + D1125A, $p=0.0003 ;$ sh-PTP $\sigma$ vs + WT, $p<0.0001$; sh-PTP $\sigma$ vs $+\Delta$ Cyto, $p>$ 0.9999; sh-PTP $\sigma$ vs $+\Delta \mathrm{D} 2, p>0.9999$; sh-PTP $\sigma$ vs + Swap, $p=0.0061$; sh-PTP $\sigma$ vs $+\mathrm{C} 1157 \mathrm{~S}, p=0.3426$; and sh-PTP $\sigma \mathrm{vs}+\mathrm{D} 1125 \mathrm{~A}, p>0.9999$. combination of extracellular signals and intracellular signaling cascades may be critical for the maintenance of excitatory synapse density in neuronal dendrites.

\section{Discussion}

\section{LAR-RPTPs control excitatory and inhibitory synapse} development in an isoform-dependent manner

The three members of the LAR-RPTP protein family are structurally similar, but their expression profiles across brain regions differ markedly (Kwon et al., 2010). Importantly, individual members of this family bind to a distinct repertoire of extracellular ligands (Um and Ko, 2013). Given the high similarity of the Ig domains among all three LAR-RPTPs, it is puzzling why various extracellular ligands bind to only a subset of LAR-RPTPs. Nevertheless, several recent studies have suggested a converging molecular model, postulating that PTP $\sigma$ is specifically involved in excitatory synapse development, whereas $\mathrm{PTP} \delta$ is generally involved in inhibitory synapse development (Um and Ko, 2013).

However, previous studies did not demonstrate a requirement for PTP $\sigma$ or PTP $\delta$ in the development of specific types of synapse in neurons. In addition, whether both synapse types involve similar vesicular packets (i.e., synaptic vesicle protein transport vesicles and/or Piccolo-bassoon transport vesicles) for the initial recruitment of nascent synaptic proteins has not been established, although both vesicular packets were reported to be similarly trapped and accumulated by trans-synaptic adhesion signaling (Bury and Sabo, 2014). In the present study, we demonstrated that PTP $\sigma$ is specifically involved in the innervation of excitatory synaptic inputs and the regulation of excitatory synaptic transmission in cultured hippocampal neurons, consistent with our previous observations (Ko et al., 2015b). Similarly, PTP $\delta$ was shown to be specifically involved in the innervation of inhibitory synaptic inputs and the regulation of inhibitory synaptic transmission (Fig. 4). An exception to this scenario arises from the observation that presynaptic PTP $\delta$ is required for excitatory heterologous synapse formation induced by IL1RAPL1, which acts specifically to regulate excitatory synapse development (Valnegri et al., 2011; Yoshida et al., 2011) (Fig. 1). A more recent study appeared to resolve this conflict, showing that IL1RAPL1 regulates dendritic morphology in cultured neurons, independently of interactions with PTP $\delta$ (Montani et al., 2017), hinting at the possibility that a minor fraction of $\mathrm{PTP} \delta$ proteins participates in excitatory synapse development. It remains to be determined when and how PTP $\delta$ is targeted to inhibitory or excitatory synaptic sites in presynaptic neurons.

\section{Various extracellular and intracellular mechanisms are involved in LAR-RPTP-mediated presynaptic assembly} To reveal key synaptogenesis mechanisms underlying retrograde trans-synaptic signaling involving LAR-RPTPs, we used heterologous synapse-formation assays in conjunction with a lentiviralmediated molecular replacement strategy using various PTPo variants (Figs. 5-10). Although the heterologous synapseformation approach used in the present study represents a reduced system, these analyses allowed us to dissect the detailed synaptogenesis mechanisms that occur in presynaptic or postsynaptic neurons during intercellular adhesion signaling. A similar approach was previously applied to identify the molecular mechanisms underlying neurexin-mediated heterologous synapse formation (Gokce and Südhof, 2013). The cytoplasmic regions of neurexins are dispensable for the recruitment of molecular machineries into nascent synaptogenic sites, although the C-terminal PDZ-binding motif of neurexins is essential for cell 

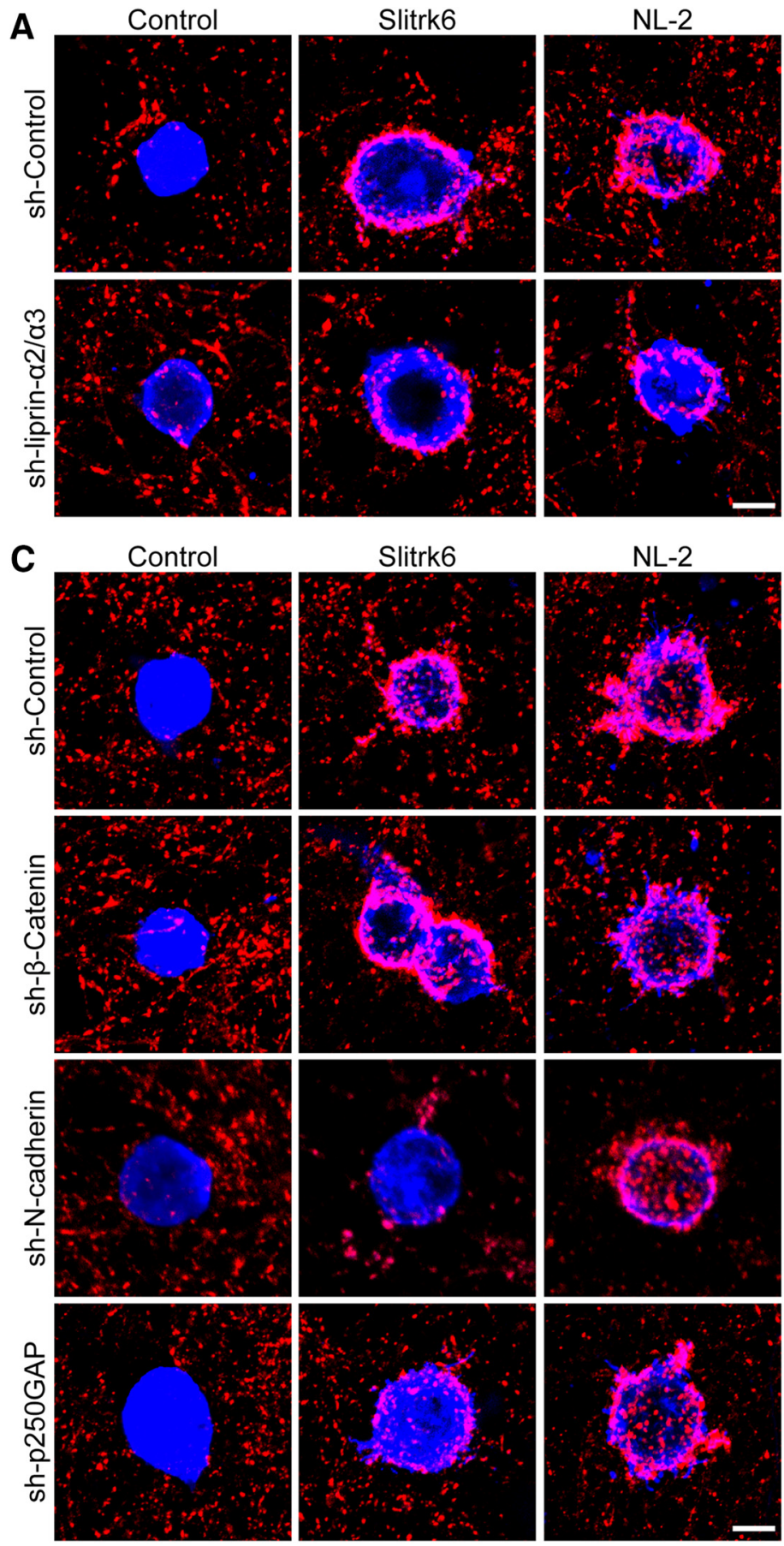

B

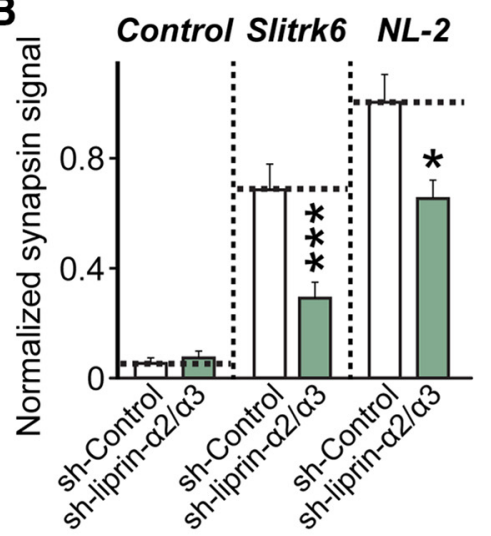

D

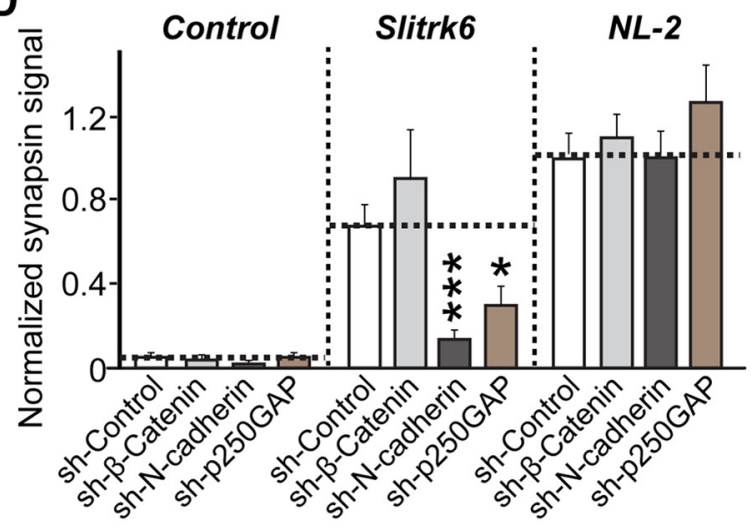

E

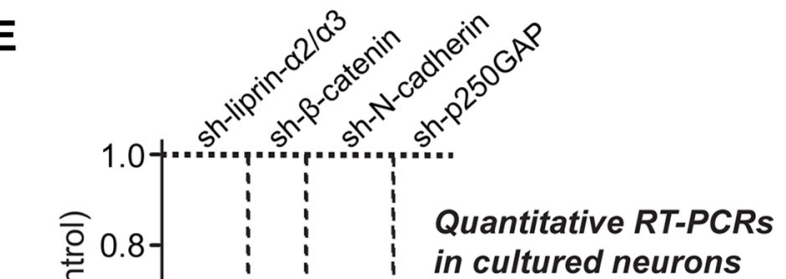

Figure 11. Effects of PTP $\sigma$ intracellular binding proteins on PTP $\sigma$-mediated heterologous synapse formation. $\boldsymbol{A}$, Representative images of the heterologous synapse-formation activities of liprin- $\alpha 2$ and liprin- $\alpha 3$. Neurons were infected at DIV4 with lentiviruses expressing sh-Control or sh-liprin- $\alpha 2 / \alpha 3$, and then cocultured from DIV9 to DIV11 with HEK293T cells expressing EGFP alone (Control), HA-Slitrk6 (Slitrk6), or HA-neuroligin-2 (NL-2). Neurons were stained with antibodies against EGFP or HA (blue) and synapsin (red). Scale bar (all images), $10 \mu \mathrm{m}$. Liprin- $\alpha 3$ and a subset of its associated proteins were isolated by mass spectroscopy (see Figure 11-1, available at https://doi.org/10.1523/JNEUROSCI.0672-18.2018.f11-1). $\boldsymbol{B}$, The synapse-formation activity in $\boldsymbol{A}$ was quantified by measuring the ratio of synapsin staining intensity (red) to HA/EGFP intensity (blue). Data are mean $\pm S E M$. Mann-Whitney $U$ test: ${ }^{*} p<0.05 ;{ }^{* * *} p<0.001 . n=$ number of neurons as follows: sh-Control/Control, $n=14$; sh-liprin- $\alpha 2 / \alpha 3 /$ Control, $n=10$; sh-Control/Slitrk6, $n=16$; sh-liprin- $\alpha 2 / \alpha 3 /$ Slitrk6, $n=15$; sh-Control/NL-2, $n=22 ;$ and sh-liprin- $\alpha 2 / \alpha 3 / \mathrm{NL}-2, n=$ 21. $p$ values for Control condition: sh-Control vs sh-liprin- $\alpha 2 / \alpha 3, p=0.2591$. $p$ values for Slitrk6 condition: sh-Control vs sh-liprin- $\alpha 2 / \alpha 3, p=0.0003$. $p$ values for NL-2 condition: sh-Control vs sh-liprin- $\alpha 2 / \alpha 3, p=0.0101$. C, Representative images of the heterologous synapse-formation activities of three selected PTP $\sigma$ substrates ( $\beta$-catenin, N-cadherin, and p250GAP). Neurons were infected at DIV4 with lentiviruses expressing sh-Control, sh- $\beta$-catenin, sh-N-cadherin, or sh-p250GAP, and then cocultured from DIV9 to DIV11 with HEK293T cells expressing EGFP alone (Control), HA-Slitrk6 (Slitrk6), or neuroligin-2-mVenus (NL-2). Neurons were stained with antibodies against EGFP or HA (blue) and synapsin (red). Scale bar (all images), $10 \mu \mathrm{m}$. D, The synapse-formation activity in $C$ was quantified by measuring the ratio of synapsin staining intensity (red) to HA/EGFP intensity (blue). Data are mean \pm SEM. Mann-Whitney $U$ test: ${ }^{*} p<0.05 ;{ }^{* * *} p<0.001 . n=$ number of neurons as follows: sh-Control/Control, $n=14$; sh- $\beta$-catenin/Control, $n=12$; sh-N-cadherin/Control, $n=10$; sh-p250GAP/Control, $n=13 ;$ sh-Control/Slitrk6, $n=16$; sh- $\beta$-catenin/ Slitrk6, $n=14 ;$ sh-N-cadherin/Slitrk6, $n=15 ;$ sh-p250GAP/Slitrk6, $n=16$; sh-Control/NL-2, $n=22 ;$ sh- $\beta$-catenin/NL-2, $n=27 ;$ sh-N-cadherin/NL-2, $n=21 ;$ and sh-p250GAP/NL-2, $n=24$. $p$ values for Control condition: sh-Control vs sh- $\beta$-catenin, $p>0.9999$; sh-Control vs sh-N-cadherin, $p=0.3904$; and sh-Control vs sh-p250GAP, $p>0.9999$. $p$ values for Slitrk6 condition: sh-Control vs sh- $\beta$-catenin, $p>0.9999$; sh-Control vs sh-N-cadherin, $p<0.0001$; and sh-Control vs sh-p250GAP, $p=0.0121$. $p$ values for NL-2 condition: sh-Control vs sh- $\beta$-catenin, $p>0.9999$; sh-Control vs sh-N-cadherin, $p>0.9999$; and sh-Control vs sh-p250GAP, $p>0.9999$. E, KD efficacies of shRNAs. Levels of target mRNAs were measured by qRT-PCR in cultured cortical neurons infected at DIV3 with lentiviruses expressing the indicated shRNAs. mRNAs were prepared at DIV12-DV13. Dotted line indicates the 70\% knockdown cutoff level for tests of biological effects. 
A

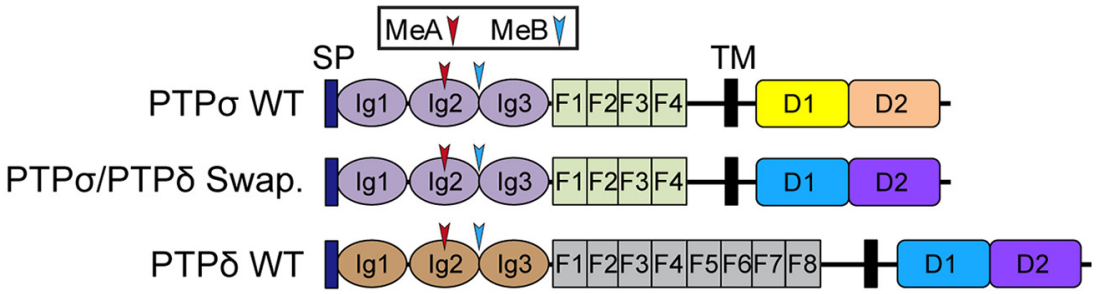

B
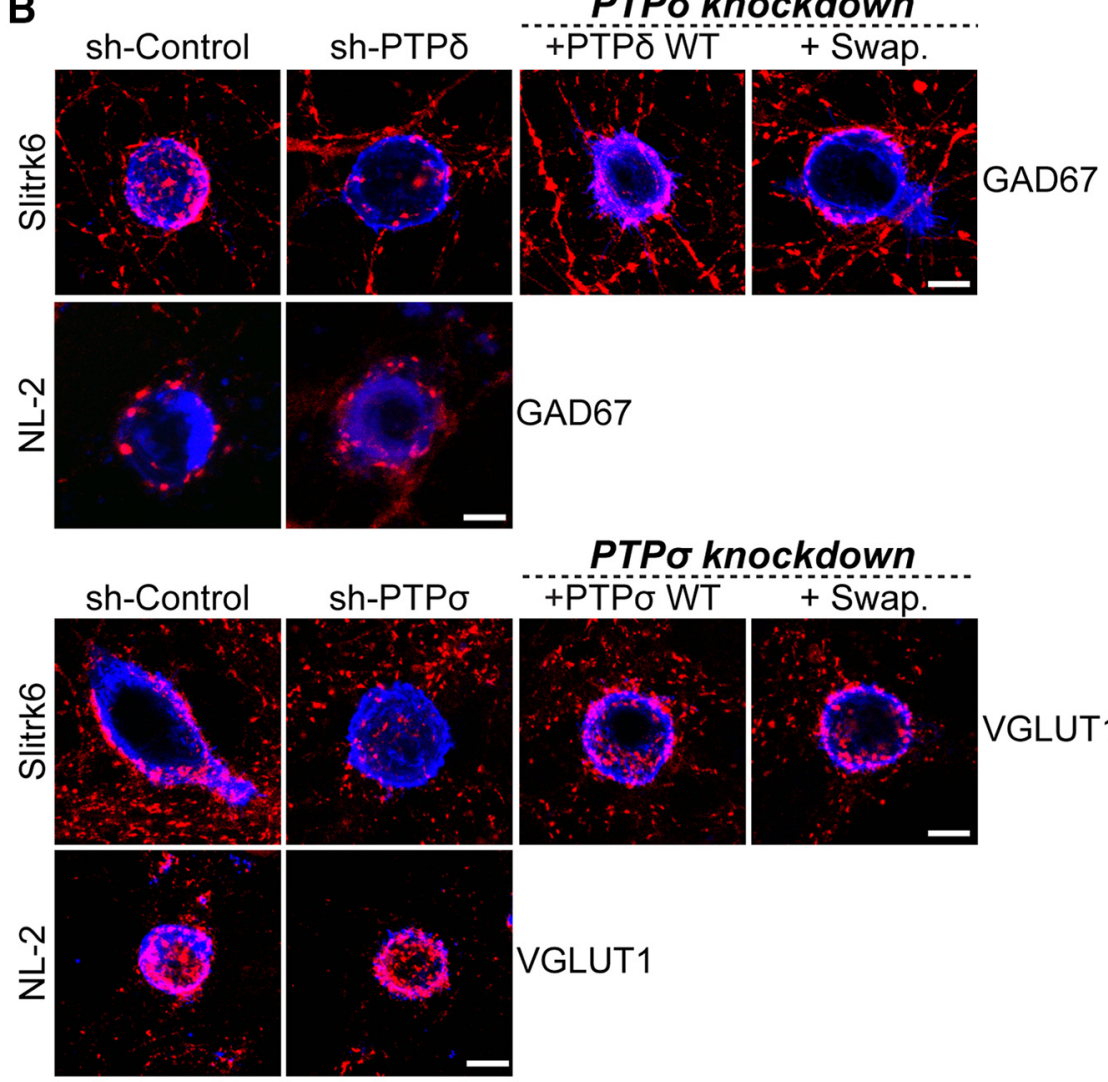

PTPo knockdown

C

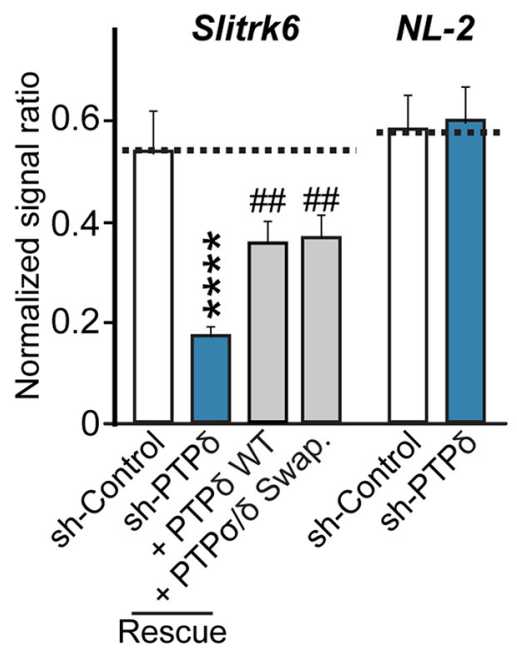

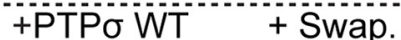

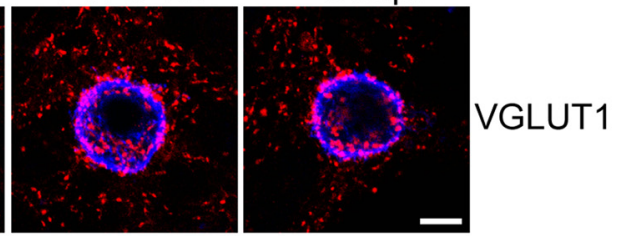

VGLUT1
D

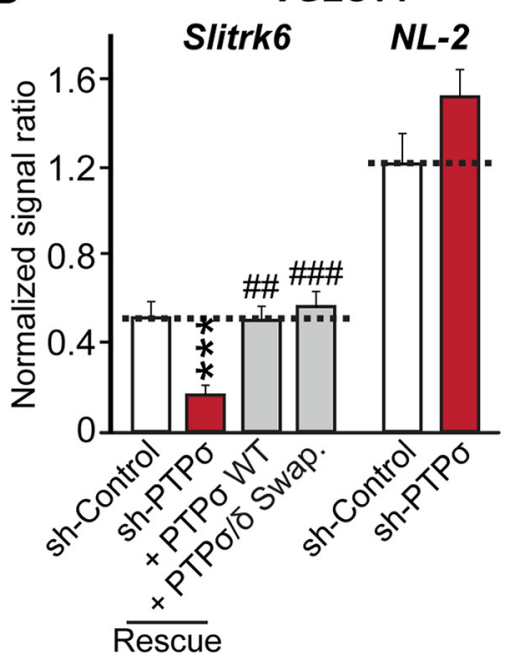

Figure 12. Effects of PTP $\sigma$ extracellular domain and PTP $\delta$ intracellular domain on PTP $\sigma$-mediated heterologous synapse formation. $\boldsymbol{A}$, Schematic illustration of PTP $\sigma$ WT and mutants used in the experiments presented in $\boldsymbol{B}-\boldsymbol{D}$. $\boldsymbol{B}$, Representative images of the heterologous excitatory or inhibitory synapse-formation activities of PTP $\sigma$ WT and PTP $\sigma /$ PTP $\delta$ Swap mutants. Neurons were infected at DIV4 with lentiviruses expressing sh-Control, sh-PTP $\sigma$, or sh-PTP $\delta$, or coexpressing sh-PTP $\sigma$ or sh-PTP $\delta$ with the indicated PTP $\sigma$ or PTP $\delta$ expression vectors, and then cocultured from DIV9 to DIV11 with HEK293T cells expressing HA-Slitrk6 (Slitrk6) or neuroligin-2 fused to mVenus (NL-2). Neurons were stained with antibodies against HA (blue) and GAD67 or VGLUT1 (red). Scale bar (all images), $10 \mu \mathrm{m}$. C, D, The synapse-formation activity in $\boldsymbol{B}$ was quantified by measuring the ratio of GAD67

surface trafficking (Gokce and Südhof, 2013). These data suggest that neurexins require coreceptor(s) in presynaptic membranes to organize the trans-synaptic signaling cascades, likely through direct interactions of neurexin coreceptor(s) with intracellular proteins in presynaptic neurons. In the current study, we asked whether LAR-RPTPs act in a manner similar to that of neurexins. We elucidated four PTP $\sigma$ regulatory mechanisms that collectively contribute to orchestrating PTP $\sigma$-mediated synaptogenic signaling at excitatory synapses (mechanisms that can possibly be extrapolated to $\mathrm{PTP} \delta$ ).

First, we evaluated the physiological significance of PTP $\sigma$ alternative splicing in cultured neurons. Alternative splicing involving insertions at the $\mathrm{MeA}$ and $\mathrm{MeB}$ splice sites of PTP $\sigma$ controls the affinity of PTP $\sigma$ for most postsynaptic ligands, but there are exceptions (e.g., GPCs) (Han et al., 2016). Indeed, reexpression of $\mathrm{PTP} \sigma^{\mathrm{MeA}+\mathrm{MeB}+}$ in PTP $\sigma$-deficient neurons rescued the abrogated heterologous synapse formation induced by Slitrk1 and TrkC (Figs. 7-10) (Um et al., 2014a). Strikingly, reexpression of $\mathrm{PTP} \sigma$ splice variants comparably rescued the decreased excitatory synaptic puncta density observed in PTP $\sigma$-deficient neurons to control levels, regardless of PTP $\sigma$ splicing status. These findings suggest that alternative splicing of PTP $\sigma$ primarily serves to select the postsynaptic ligand that organizes the specific trans-synaptic complex, whereas alternative splicing-independent mechanisms are involved in regulating PTP $\sigma$-mediated excitatory synapse development (Fig. 5).

\footnotetext{
(C) or VGLUT1 (D) staining intensity (red) to HA/EGFP intensity (blue). Data are mean \pm SEM. ANOVA with a nonparametric Kruskal-Wallis test: ${ }^{* *} p<0.001 ;{ }^{* * * *} p<0.0001 ;{ }^{\# \#} p<$ $0.01 ;{ }^{\# \#} p<0.001 . n=$ number of neurons as follows: shControl/GAD67/Slitrk6, $n=16$; sh-PTP8/GAD67/Slitrk6, $n=$ 15; + PTP $\delta /$ GAD67/Slitrk6, $n=16$; + PTP $\sigma$ Swap/GAD67/ Slitrk6, $n=17$; sh-Control/VGLUT1/Slitrk6, $n=15$; sh-PTP $\sigma /$ VGLUT1/Slitrk6, $n=15 ;+$ PTP $\sigma$ WT/VGLUT1/Slitrk6, $n=$ $12 ;+$ PTP $\sigma$ Swap/VGLUT1/Slitrk6, $n=13$; sh-Control/ GAD67/NL-2, $n=21$; sh-PTP $\delta /$ GAD67/NL-2, $n=23$; shControl/VGLUT1/NL-2, $n=12$; and sh-PTP $\sigma /$ VGLUT1/NL-2, $n=11 . p$ values for Slitrk6/GAD67 condition: sh-Control vs sh-PTP $\delta, p<0.0001$; sh-Control vs + PTP $\delta, p=0.4456$; sh-Control vs + PTP $\sigma$ Swap, $p=0.4583$; sh-PTP $\delta$ vs + PTP $\delta, p=0.0064$; and sh-PTP $\delta$ vs + PTP $\sigma$ Swap, $p=$ 0.0047. $p$ values for Slitrk6/VGLUT1 condition: sh-Control vs sh-PTP $\sigma, p=0.0004$; sh-Control vs + PTP $\sigma, p>0.9999$; sh-control vs + PTP $\sigma$ Swap, $p>0.9999$; sh-PTP $\sigma$ vs + PTP $\sigma, p=0.0019$; and sh-PTP $\sigma$ vs + PTP $\sigma$ Swap, $p=$ 0.0002. $p$ values for NL-2/GAD67 condition: sh-Control vs shPTP $\delta, p>0.9999 . p$ values for NL-2/VGLUT1 condition: shControl vs sh-PTP $\sigma, p>0.9999$.
} 
A extracellualr and intracellular
signaling model

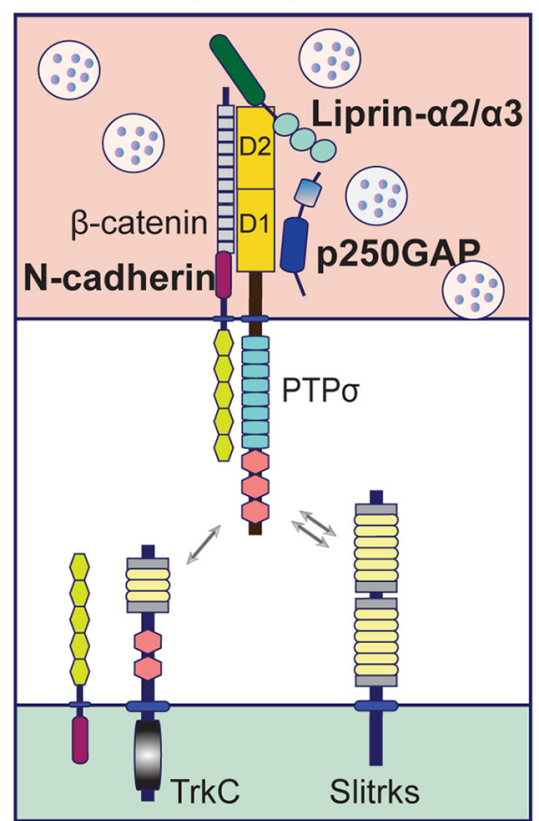

B
Neurexin-based trans-synaptic signaling model

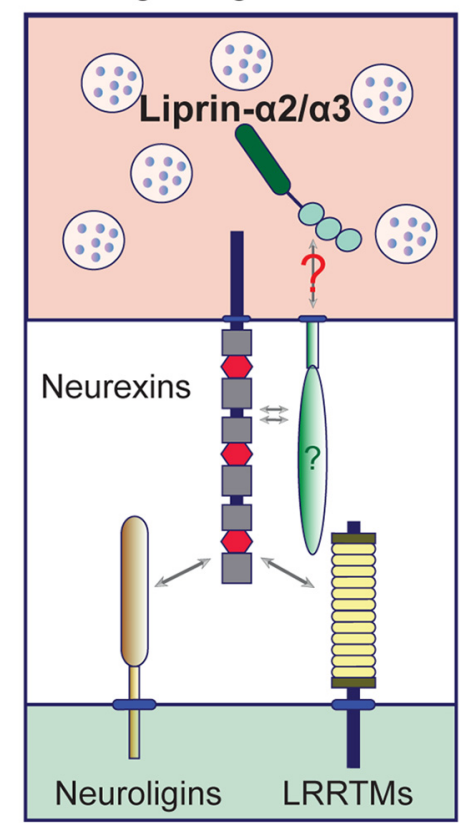

Figure 13. Molecular model of PTP $\sigma$ signaling pathways in heterologous synapse formation. $\boldsymbol{A}, \mathrm{PTP} \sigma$ triggers excitatory heterologous synapse formation through a combination of extracellular and intracellular signaling components. The PTP $\sigma$ D2 domain binds intracellular adaptor proteins (e.g., liprin- $\alpha$ ) and substrates (e.g., p250GAP and N-cadherin) to recruit the vesicular machinery for excitatory synapse development. This signal transduction model differs from that of neurexin (see below). $\boldsymbol{B}$, Neurexins serve as anchor proteins that transduce postsynaptic signals from various ligands (e.g., neuroligins and LRRTM proteins) and transfer them to adjacent, but as yet unidentified, coreceptor protein(s) to mediate the signal transduction cascades necessary for full heterologous synapse formation activity (Gokce and Südhof, 2013).

Second, we assessed the effects of PTP $\sigma$ interactions with a subset of ligands (Slitrks, TrkC, and GPCs). We found that reexpression of PTP $\sigma$ Y233S or R235D (but not R97A/R100A) failed to rescue the impaired excitatory synapse development observed in PTP $\sigma$-deficient neurons, suggesting that PTP $\sigma /$ Slitrkmediated synaptic adhesion pathways may be more central than other PTP $\sigma$-mediated synaptic adhesion pathways in maintaining excitatory synapse development. Additional structural information obtained from structures of complexes involving PTP $\sigma$ and other ligands (GPCs or SALMs) should provide additional mechanistic insights into PTP $\sigma$-mediated excitatory synapse development.

Third, we confirmed the contribution of the HS-binding feature of PTP $\sigma$ in regulating excitatory synapse development (Fig. 7), as previously reported (Ko et al., 2015b). HS chains contribute to the assembly of PTP $\sigma$ oligomers (Coles et al., 2011) and compete with TrkC for PTP $\sigma$ binding, reflecting the partially overlapping binding interface of TrkC with that of HS (Coles et al., 2014). Thus, it is possible to postulate that HS-bound oligomeric PTP $\sigma$ proteins form specific complexes with GPCs, and HS-unbound monomeric PTP $\sigma$ proteins form specific associations with TrkC (Han et al., 2016). Indeed, reexpression of an HS-bindingdefective PTP $\sigma$ [AAAA] mutant in PTP $\sigma$-deficient neurons rescued impaired TrkC-induced heterologous synapse formation (Fig. 10). Reexpression of the PTP $\sigma$ [AAAA] mutant in PTP $\sigma$ deficient neurons also rescued the impaired Slitrk1-induced heterologous synapse formation (Fig. 7), suggesting that HS chains serve a regulatory function that promotes preferential binding of monomeric PTP $\sigma$ to TrkC and Slitrks instead of GPCs (Won et al., 2017).
Fourth, we identified a key intracellular mechanism underlying PTP $\sigma$ mediated synaptic signaling in presynaptic neurons (Figs. 9-11). We demonstrated a requirement for D2 domain-mediated recruitment of a subset of known PTP $\sigma$ substrates in the vicinity of PTP $\sigma$. Although our data suggested that the phosphatase activity of PTP $\sigma$ is not fully required for PTP $\sigma$-mediated heterologous synapse formation (Figs. 9, 10), p250GAP and $\mathrm{N}$-cadherin, but not $\beta$-catenin, were necessary for PTP $\sigma$-mediated heterologous synapse formation (Fig. 11). Because postsynaptic $\beta$-catenin and $\mathrm{N}$-cadherin are required for LAR-RPTP-mediated targeting of AMPA receptors to postsynaptic sites and regulating dendritic spines (Dunah et al., 2005; Saglietti et al., 2007), as well as controlling neurotransmitter release (Vitureira et al., 2011), both of which may involve tyrosine phosphorylation signaling pathways, differential sets of downstream mechanisms in both presynaptic and postsynaptic neurons may participate in distinct aspects of synaptogenesis. Other PTP $\sigma$ substrates that were not tested in the current study include Abl, Ena, and Trio, all of which are known to be involved in actin polymerization, similarly to p250GAP (Lanier and Gertler, 2000; Bateman et al., 2001; Miller et al., 2013). Thus, it is tempting to speculate that regulation of the actin cytoskeleton by Rac signaling is an important step in PTP $\sigma$-mediated heterologous synapse formation in presynaptic neurons. This suggested role is unique in that actin is not enriched in the course of heterologous synapse formation mediated by presynaptic neurexins (Gokce and Südhof, 2013). Given that local assembly of an F-actin cytoskeleton at nascent presynaptic sites links synaptic adhesion events with axonal branch formation (Chia et al., 2014), subsequent studies are warranted to further elucidate LARRPTP-associated presynaptic assembly and their various postsynaptic ligands. In particular, how HS-binding-induced oligomerization of PTP $\sigma$ might influence intracellular signaling cascades by triggering conformational changes, given the flexibility of the PTP $\sigma$ structure (Coles et al., 2014), remains to be determined. In addition, proteomic analyses to identify the intracellular component of LAR-RPTP complex in vivo are required for accurately mapping of additional key components in presynaptic neurons, as recently attempted (Loh et al., 2016; Uezu et al., 2016) (Fig. 13). Considering the possibility that coreceptor(s) of neurexins converge on intracellular signaling pathways that overlap with components linked to LAR-RPTP-mediated signaling cascades (e.g., liprin- $\alpha 2 / 3$ ), profiling the complete collection of scaffolds in presynaptic neurons will facilitate our understanding of how trans-synaptic signals organize various aspects of presynaptic function.

\section{References}

Aricescu AR, McKinnell IW, Halfter W, Stoker AW (2002) Heparan sulfate proteoglycans are ligands for receptor protein tyrosine phosphatase sigma. Mol Cell Biol 22:1881-1892. CrossRef Medline 
Bateman J, Reddy RS, Saito H, Van Vactor D (2001) The receptor tyrosine phosphatase dlar and integrins organize actin filaments in the Drosophila follicular epithelium. Curr Biol 11:1317-1327. CrossRef Medline

Boucard AA, Ko J, Südhof TC (2012) High affinity neurexin binding to cell adhesion G-protein-coupled receptor CIRL1/latrophilin-1 produces an intercellular adhesion complex. J Biol Chem 287:9399-9413. CrossRef Medline

Bresler T, Shapira M, Boeckers T, Dresbach T, Futter M, Garner CC, Rosenblum K, Gundelfinger ED, Ziv NE (2004) Postsynaptic density assembly is fundamentally different from presynaptic active zone assembly. J Neurosci 24:1507-1520. CrossRef Medline

Bury LA, Sabo SL (2014) Dynamic mechanisms of neuroligin-dependent presynaptic terminal assembly in living cortical neurons. Neural Dev 9:13. CrossRef Medline

Bury LA, Sabo SL (2016) Building a terminal: mechanisms of presynaptic development in the CNS. Neuroscientist 22:372-391. CrossRef Medline

Chia PH, Chen B, Li P, Rosen MK, Shen K (2014) Local F-actin network links synapse formation and axon branching. Cell 156:208-220. CrossRef Medline

Choi Y, Nam J, Whitcomb DJ, Song YS, Kim D, Jeon S, Um JW, Lee SG, Woo J, Kwon SK, Li Y, Mah W, Kim HM, Ko J, Cho K, Kim E (2016) SALM5 trans-synaptically interacts with LAR-RPTPs in a splicing-dependent manner to regulate synapse development. Sci Rep 6:26676. CrossRef Medline

Coles CH, Shen Y, Tenney AP, Siebold C, Sutton GC, Lu W, Gallagher JT, Jones EY, Flanagan JG, Aricescu AR (2011) Proteoglycan-specific molecular switch for RPTPsigma clustering and neuronal extension. Science 332:484-488. CrossRef Medline

Coles CH, Mitakidis N, Zhang P, Elegheert J, Lu W, Stoker AW, Nakagawa T, Craig AM, Jones EY, Aricescu AR (2014) Structural basis for extracellular cis and trans RPTPsigma signal competition in synaptogenesis. Nat Commun 5:5209. CrossRef Medline

Dunah AW, Hueske E, Wyszynski M, Hoogenraad CC, Jaworski J, Pak DT, Simonetta A, Liu G, Sheng M (2005) LAR receptor protein tyrosine phosphatases in the development and maintenance of excitatory synapses. Nat Neurosci 8:458-467. CrossRef Medline

Gokce O, Südhof TC (2013) Membrane-tethered monomeric neurexin LNS-domain triggers synapse formation. J Neurosci 33:14617-14628. CrossRef Medline

Han KA, Jeon S, Um JW, Ko J (2016) Emergent synapse organizers: LARRPTPs and their companions. Int Rev Cell Mol Biol 324:39-65. CrossRef Medline

Jin Y, Garner CC (2008) Molecular mechanisms of presynaptic differentiation. Annu Rev Cell Dev Biol 24:237-262. CrossRef Medline

Johnson KG, Van Vactor D (2003) Receptor protein tyrosine phosphatases in nervous system development. Physiol Rev 83:1-24. CrossRef Medline

Kang H, Han KA, Won SY, Kim HM, Lee YH, Ko J, Um JW (2016) Slitrk missense mutations associated with neuropsychiatric disorders distinctively impair slitrk trafficking and synapse formation. Front Mol Neurosci 9:104. CrossRef Medline

Ko J, Choii G, Um JW (2015a) The balancing act of GABAergic synapse organizers. Trends Mol Med 21:256-268. CrossRef Medline

Ko JS, Pramanik G, Um JW, Shim JS, Lee D, Kim KH, Chung GY, Condomitti G, Kim HM, Kim H, de Wit J, Park KS, Tabuchi K, Ko J (2015b) PTPsigma functions as a presynaptic receptor for the glypican-4/LRRTM4 complex and is essential for excitatory synaptic transmission. Proc Natl Acad Sci U S A 112:1874-1879. CrossRef Medline

Ko J, Soler-Llavina GJ, Fuccillo MV, Malenka RC, Südhof TC (2011) Neuroligins/LRRTMs prevent activity- and $\mathrm{Ca}^{2+} /$ calmodulin-dependent synapse elimination in cultured neurons. J Cell Biol 194:323-334. CrossRef Medline

Kwon SK, Woo J, Kim SY, Kim H, Kim E (2010) Trans-synaptic adhesions between netrin-G ligand-3 (NGL-3) and receptor tyrosine phosphatases LAR, protein-tyrosine phosphatase delta (PTPdelta), and PTPsigma via specific domains regulate excitatory synapse formation. J Biol Chem 285: 13966-13978. CrossRef Medline

Lanier LM, Gertler FB (2000) From abl to actin: abl tyrosine kinase and associated proteins in growth cone motility. Curr Opin Neurobiol 10:8087. CrossRef Medline

Li Y, Zhang P, Choi TY, Park SK, Park H, Lee EJ, Lee D, Roh JD, Mah W, Kim R, Kim Y, Kwon H, Bae YC, Choi SY, Craig AM, Kim E (2015) Splicing- dependent trans-synaptic SALM3-LAR-RPTP interactions regulate excitatory synapse development and locomotion. Cell Rep 12:1618-1630. CrossRef Medline

Loh KH, Stawski PS, Draycott AS, Udeshi ND, Lehrman EK, Wilton DK, Svinkina T, Deerinck TJ, Ellisman MH, Stevens B, Carr SA, Ting AY (2016) Proteomic analysis of unbounded cellular compartments: synaptic clefts. Cell 166:1295-1307.e21. CrossRef Medline

Maas C, Torres VI, Altrock WD, Leal-Ortiz S, Wagh D, Terry-Lorenzo RT, Fejtova A, Gundelfinger ED, Ziv NE, Garner CC (2012) Formation of Golgi-derived active zone precursor vesicles. J Neurosci 32:11095-11108. CrossRef Medline

McAllister AK (2007) Dynamic aspects of CNS synapse formation. Annu Rev Neurosci 30:425-450. CrossRef Medline

Miller MB, Yan Y, Eipper BA, Mains RE (2013) Neuronal rho GEFs in synaptic physiology and behavior. Neuroscientist 19:255-273. CrossRef Medline

Missler M, Südhof TC, Biederer T (2012) Synaptic cell adhesion. Cold Spring Harb Perspect Biol 4:a005694. CrossRef Medline

Montani C, Ramos-Brossier M, Ponzoni L, Gritti L, Cwetsch AW, Braida D, Saillour Y, Terragni B, Mantegazza M, Sala M, Verpelli C, Billuart P, Sala C (2017) The X-linked intellectual disability protein IL1RAPL1 regulates dendrite complexity. J Neurosci 37:6606-6627. CrossRef Medline

Nakazawa T, Kuriu T, Tezuka T, Umemori H, Okabe S, Yamamoto T (2008) Regulation of dendritic spine morphology by an NMDA receptorassociated Rho GTPase-activating protein, p250GAP. J Neurochem 105: 1384-1393. CrossRef Medline

Pang ZP, Cao P, Xu W, Südhof TC (2010) Calmodulin controls synaptic strength via presynaptic activation of calmodulin kinase II. J Neurosci 30:4132-4142. CrossRef Medline

Pulido R, Serra-Pagès C, Tang M, Streuli M (1995) The LAR/PTP delta/PTP sigma subfamily of transmembrane protein-tyrosine-phosphatases: multiple human LAR, PTP delta, and PTP sigma isoforms are expressed in a tissue-specific manner and associate with the LAR-interacting protein LIP.1. Proc Natl Acad Sci U S A 92:11686-11690. CrossRef Medline

Saglietti L, Dequidt C, Kamieniarz K, Rousset MC, Valnegri P, Thoumine O, Beretta F, Fagni L, Choquet D, Sala C, Sheng M, Passafaro M (2007) Extracellular interactions between GluR2 and N-cadherin in spine regulation. Neuron 54:461-477. CrossRef Medline

Spangler SA, Hoogenraad CC (2007) Liprin-alpha proteins: scaffold molecules for synapse maturation. Biochem Soc Trans 35:1278-1282. CrossRef Medline

Suarez F, Thostrup P, Colman D, Grutter P (2013) Dynamics of presynaptic protein recruitment induced by local presentation of artificial adhesive contacts. Dev Neurobiol 73:98-106. CrossRef Medline

Südhof TC (2008) Neuroligins and neurexins link synaptic function to cognitive disease. Nature 455:903-911. CrossRef Medline

Südhof TC (2012) The presynaptic active zone. Neuron 75:11-25. CrossRef Medline

Südhof TC (2017) Synaptic neurexin complexes: a molecular code for the logic of neural circuits. Cell 171:745-769. CrossRef Medline

Takahashi H, Arstikaitis P, Prasad T, Bartlett TE, Wang YT, Murphy TH, Craig AM (2011) Postsynaptic TrkC and presynaptic PTPsigma function as a bidirectional excitatory synaptic organizing complex. Neuron 69:287-303. CrossRef Medline

Takahashi H, Katayama K, Sohya K, Miyamoto H, Prasad T, Matsumoto Y, Ota M, Yasuda H, Tsumoto T, Aruga J, Craig AM (2012) Selective control of inhibitory synapse development by Slitrk3-PTPdelta transsynaptic interaction. Nat Neurosci 15:389-398, S1-S2. CrossRef Medline

Uezu A, Kanak DJ, Bradshaw TW, Soderblom EJ, Catavero CM, Burette AC, Weinberg RJ, Soderling SH (2016) Identification of an elaborate complex mediating postsynaptic inhibition. Science 353:1123-1129. CrossRef Medline

Um JW, Ko J (2013) LAR-RPTPs: synaptic adhesion molecules that shape synapse development. Trends Cell Biol 23:465-475. CrossRef Medline

Um JW, Kim KH, Park BS, Choi Y, Kim D, Kim CY, Kim SJ, Kim M, Ko JS, Lee SG, Choii G, Nam J, Heo WD, Kim E, Lee JO, Ko J, Kim HM (2014a) Structural basis for LAR-RPTP/Slitrk complex-mediated synaptic adhesion. Nat Commun 5:5423. CrossRef Medline

Um JW, Pramanik G, Ko JS, Song MY, Lee D, Kim H, Park KS, Südhof TC, Tabuchi K, Ko J (2014b) Calsyntenins function as synaptogenic adhe- 
sion molecules in concert with neurexins. Cell Rep 6:1096-1109. CrossRef Medline

Valnegri P, Montrasio C, Brambilla D, Ko J, Passafaro M, Sala C (2011) The $\mathrm{X}$-linked intellectual disability protein IL1RAPL1 regulates excitatory synapse formation by binding PTPdelta and RhoGAP2. Hum Mol Genet 20:4797-4809. CrossRef Medline

Vitureira N, Letellier M, White IJ, Goda Y (2011) Differential control of presynaptic efficacy by postsynaptic $\mathrm{N}$-cadherin and beta-catenin. Nat Neurosci 15:81-89. CrossRef Medline

Won SY, Kim CY, Kim D, Ko J, Um JW, Lee SB, Buck M, Kim E, Heo WD, Lee JO, Kim HM (2017) LAR-RPTP clustering is modulated by competitive binding between synaptic adhesion partners and heparan sulfate. Front Mol Neurosci 10:327. CrossRef Medline

Yim YS, Kwon Y, Nam J, Yoon HI, Lee K, Kim DG, Kim E, Kim CH, Ko J
(2013) Slitrks control excitatory and inhibitory synapse formation with LAR receptor protein tyrosine phosphatases. Proc Natl Acad Sci U S A 110:4057-4062. CrossRef Medline

Yoshida T, Yasumura M, Uemura T, Lee SJ, Ra M, Taguchi R, Iwakura Y, Mishina M (2011) IL-1 receptor accessory protein-like 1 associated with mental retardation and autism mediates synapse formation by transsynaptic interaction with protein tyrosine phosphatase delta. J Neurosci 31:13485-13499. CrossRef Medline

Ziv NE, Garner CC (2004) Cellular and molecular mechanisms of presynaptic assembly. Nat Rev Neurosci 5:385-399. CrossRef Medline

Zürner M, Mittelstaedt T, tom Dieck S, Becker A, Schoch S (2011) Analyses of the spatiotemporal expression and subcellular localization of liprin-alpha proteins. J Comp Neurol 519:3019-3039. CrossRef Medline 